\title{
Electron-translation effects in heavy-ion scattering
}

\author{
Ulrich Heinz and Walter Greiner* \\ Wright Nuclear Structure Laboratory, Yale University, New Haven, Connecticut 06511 \\ Berndt Müller \\ Institut für Theoretische Physik, J. W. Goethe-Universität, Frankfurt am Main, West Germany
}

(Received 3 June 1980)

\begin{abstract}
The origin and importance of electron-translation effects within a molecular description of electronic excitations in heavy-ion collisions is investigated. First, a fully consistent quantum-mechanical description of the scattering process is developed; the electrons are described by relativistic molecular orbitals, while the nuclear motion is approximated nonrelativistically. Leaving the quantum-mechanical level by using the semiclassical approximation for the nuclear motion, a set of coupled differential equations for the occupation amplitudes of the molecular orbitals is derived. In these coupled-channel equations the spurious asymptotic dynamical couplings are corrected for by additional matrix elements stemming from the electron translation. Hence, a molecular description of electronic excitations in heavy-ion scattering has been achieved, which is free from the spurious asymptotic couplings of the conventional perturbated stationary-state approach. The importance of electron-translation effects for continuum electrons and positrons is investigated. To this end an algorithm for the description of continuum electrons is proposed, which for the first time should allow for the calculation of angular distributions for $\delta$ electrons. Finally, the practical consequences of electron-translation effects are studied by calculating the corrected coupling matrix elements for the $\mathrm{Pb}-\mathrm{Cm}$ system and comparing the corresponding $K$-vacancy probabilities with conventional calculations. We critically discuss conventional methods for cutting off the coupling matrix elements in coupledchannel calculations.
\end{abstract}

\section{INTRODUCTION}

During the last years a widespread interest has developed concerning the excitations of the electronic shells in collisions of very heavy ions and atoms. The reason for that is that those excitation cross sections allow us to study the behavior of electrons in the very strong electromagnetic field of two highly charged nuclei approaching each other up to a few femtometers. At the point of closest approach of, for example, two uranium nuclei, the electrons of the inner shells for a short time feel a strong quasimolecular field created by two sources with total charge $Z_{\text {tot }}$ $=184$. This has a large influence on the electronic charge distribution and binding energies. The inner-shell-electron wave functions are strongly contracted, their binding energies strongly increase, so that the orbital velocity of those states becomes nearly equal to the velocity of light, and the binding energy can reach values of the order of or even larger than twice the electron rest mass. ${ }^{1}$ Because of the large orbital velocity compared to the relative velocity of the nuclei in the scattering process, the inner-shell electrons behave to a good degree of approximation adiabatically ${ }^{2}$ and form quasimolecular orbitals. ${ }^{3,4,5}$ Signatures for the development of molecular orbitals have been experimentally found by studying the $\mathrm{x}$-ray spectra. ${ }^{6-11} \mathrm{~A}$ unique signature for the existence of $K$-shell binding energies larger than $2 m_{0} c^{2}$ in collisions with total charge $Z_{\text {tot }}>173$ (Refs. 4 and 12) would be the detection of the decay of the neutral vacuum, ${ }^{13}$ i.e., the spontaneous filling of a $K$ hole followed by emission of a positron ("spontaneous positron production"). However, due to the dynamics of the problem and the large kinetic energy of the nuclear relative motion, many other processes occur which eventually lead to positron production. ${ }^{1,14}$ Therefore, separation of the contribution by the spontaneously produced positrons would require an exact knowledge of all other excitation processes within the electron shell during the collision.

To this end in the last years extensive calculations have been done ${ }^{14,15,16}$ where the excitations (including molecular radiative transitions) have been calculated by solving a set of coupled differential equations for the occupation amplitudes of the electron states. These coupled-channel equations are obtained by first decoupling the nuclear motion by treating it semiclassically. Then a time-dependent Dirac equation for the electrons remains to be solved, the time dependence stemming from the time dependence of the internuclear distance given by the classical trajectory $\vec{R}(t)$ :

$$
\frac{i}{\hbar} \frac{\partial \psi}{\partial t}(\overrightarrow{\mathrm{r}}, t)=\hat{H}_{\mathrm{TCD}}(\overrightarrow{\mathrm{r}}, t) \psi(\overrightarrow{\mathrm{r}}, t),
$$

where

$$
\hat{H}_{\mathrm{TCD}}(\overrightarrow{\mathrm{r}}, t) \equiv \hat{H}_{\mathrm{TCD}}(\overrightarrow{\mathrm{r}}, \overrightarrow{\mathrm{R}}(t))
$$


is the two-center-Dirac (TCD) Hamiltonian for the two nuclei being at a distance $\vec{R}(t)$. Expanding the wave function $\psi(\vec{r}, t)$ into the adiabatic molecular basis $\varphi_{n}(\overrightarrow{\mathrm{r}}, \overrightarrow{\mathrm{R}})$ solving

$$
\begin{aligned}
& \hat{H}_{\mathrm{TCD}}(\overrightarrow{\mathrm{r}}, \overrightarrow{\mathrm{R}}) \varphi_{n}(\overrightarrow{\mathrm{r}}, \overrightarrow{\mathrm{R}})=\epsilon_{n}(R) \varphi_{n}(\overrightarrow{\mathrm{r}}, \overrightarrow{\mathrm{R}}) \\
& \dot{a}_{n}(t)=-\sum_{m}\left\langle\varphi_{n}\left|\frac{\partial}{\partial t}\right| \varphi_{m}\right\rangle \exp \left(\frac{i}{\hbar} \int_{0}^{t}\left[\epsilon_{n}\left(t^{\prime}\right)-\epsilon_{m}\left(t^{\prime}\right)\right] d t^{\prime}\right) a_{m}(t) \\
& =-\sum_{m}\left\langle\varphi_{n}\left|\dot{R} \partial_{R}-\frac{i}{\hbar} \omega_{y} J_{y}\right| \varphi_{m}\right\rangle \exp \left(\frac{i}{\hbar} \int_{0}^{t}\left(\epsilon_{n}-\epsilon_{m}\right) d t^{\prime}\right) a_{m}(t)
\end{aligned}
$$

by the ansatz

$$
\psi(\overrightarrow{\mathrm{r}}, t)=\sum_{n} a_{n}(t) \varphi_{n}(\overrightarrow{\mathrm{r}}, \overrightarrow{\mathrm{R}}(t)) \exp \left(-\frac{i}{\hbar} \int_{0}^{t} \epsilon_{n}\left(R\left(t^{\prime}\right)\right) d t^{\prime}\right)
$$

yields the well-known set of coupled-channel equations for the $a_{n}(t)$ :
Here the $\theta / \partial t$ coupling has been rewritten via the classical trajectory $\vec{R}(t)$ into a radial coupling $\dot{R} \partial / \partial R$ and a rotational coupling $i / \hbar \omega_{y} J_{y}$ (where $J$ is the electronic angular-momentum operator). Cross sections may be calculated in terms of $\left|a_{n}(\infty)\right|^{2}$, the asymptotic probability to find the electronic shell in state $\left|\varphi_{n}\right\rangle$.

Unfortunately, this generally used method has a major disadvantage: the $\partial / \partial t$ coupling between the molecular states $\varphi_{n}$ does not vanish as $R-\infty$. Hence the asymptotic occupation probabilities $\left|a_{n}(\infty)\right|^{2}$ are ill defined. However, these asymptotic $\partial / \partial t$ couplings are clearly unphysical, as the interaction between the two ions vanishes asymptotically. The reason for the spurious couplings was recognized already more than 20 years ago by Bates and McCarroll, ${ }^{17}$ who realized that they are due to the fact that the adiabatic molecular basis states are calculated keeping the relative internuclear distance $R$ fixed, whence the asymptotic translation of the electrons with the escaping nuclei is not contained in this basis. There have been numerous attempts to solve this problem $^{17-20}$ using so-called classical electron-translation factors (ETF) by which the molecular states were multiplied. However, all these attempts had serious defects (see Ref. 20 for a discussion), and only a new approach by Thorson and Delos, ${ }^{20}$ who gave up the ETF idea in favor of a nonlinear coordinate transformation leading to an electrontranslation operator instead, finally led to a satisfactory treatment of the problem.

In Sec. II we will review, slightly correct, and extend the work by Thorson and Delos ${ }^{20}$ to systems where the electrons must be described relativistically. No specification to one electron system will be made when setting up the theory. By semiclassical approximation of the nuclear motion (Sec. III) we will derive a new set of coupled-channel equations for the occupation amplitudes, which no longer contain any spurious asymptotic couplings and thus overcome the de- fects of Eq. (1.4). In Sec. IV we attack the problem of describing relativistic continuum electrons in the field of two nuclei. Since the continuum solutions of the two-center Dirac equations are not known, and even if we knew them our electron-translation-operator formalism could not succeed in asymptotically suppressing the continuum-continuum coupling, we circumvent this problem by constructing another continuum basis. This basis consists of wave packets having the necessary localization properties to ensure the asymptotic vanishing of all coupling matrix elements. It allows for a description of all electrons (and positrons) with kinetic energy large enough to be able to leave the two nuclei within a time interval comparable to the collision time $\left(\sim 10^{-19} \mathrm{sec}\right)$. Thus we suggest a theory which for the first time should allow us to calculate angular distributions of $\delta$ electrons and positrons in collisions of very heavy systems.

In Sec. $V$ we specify the switching function $f(\vec{r}, \vec{R})$ used for the actual calculation of the corrected matrix elements in the coupled-channel equations. In Sec. VI finally we present results which show the influence of electron-translation effects on the $K$-hole amplitude in $\mathrm{Pb}-\mathrm{Cm}$ collisions. Our main result will be that the natural cutoff of the $\partial / \partial t$ matrix elements by the electrontranslation matrix elements takes place much farther outside (i.e., at much larger internuclear distances) than previously ${ }^{5,50}$ assumed.

\section{QUANTUM-MECHANICAL FORMULATION OF THE PROBLEM}

We want to describe a system of two nuclei (mass $M_{A}, M_{B} \leqslant M_{A}$; charge $Z_{A}, Z_{B}$ ) and $Z^{\prime}$ electrons. In Table I we define the reduced masses and coordinates of importance for the following. Some of the them are depicted in Fig. 1. The mass asymmetry $\lambda$ satisfies the following identities: define $f_{A}=-1, f_{B}=1$, then we have 
TABLE I. Definitions of reduced masses and coordinates.

\begin{tabular}{|c|c|c|c|}
\hline \multicolumn{2}{|l|}{ Coordinates } & \multicolumn{2}{|c|}{ Corresponding masses } \\
\hline $\overrightarrow{\mathbf{r}}_{i}^{0}$ & $\begin{array}{l}\text { Coord. of electron } i \text { in the } \\
\text { laboratory system } L\end{array}$ & $m_{0}$ & Electron rest mass \\
\hline $\overrightarrow{\mathrm{R}}_{A(B)}^{0}$ & Coord. of nucleus $A(B)$ in $L$ & $M_{A(B)}$ & Rest mass of nucleus $A(B)$ \\
\hline $\overrightarrow{\mathrm{R}}_{\mathrm{CMN}}=\frac{1}{M_{N}}\left(M_{A} \overrightarrow{\mathrm{R}}_{A}^{0}+M_{B} \overrightarrow{\mathrm{R}}_{B}^{0}\right)$ & $\begin{array}{l}\text { Coord. of center of mass of the } \\
\text { nuclei }(C M N) \text { in } L\end{array}$ & $M_{N}=M_{A}+M_{B}$ & Total nuclear mass \\
\hline $\overrightarrow{\mathrm{R}}_{C M}^{0}=\frac{1}{M_{T}}\left(M_{A} \overrightarrow{\mathrm{R}}_{A}^{0}+M_{B} \overrightarrow{\mathrm{R}}_{B}^{0}+m_{0} \sum_{i=1}^{c} \overrightarrow{\mathrm{r}}_{i}^{0}\right)$ & Coord. of CM in $L$ & $M_{T}=M_{A}+M_{B}+Z^{\prime} m_{0}$ & Total mass of the system \\
\hline $\overrightarrow{\mathrm{R}}=\overrightarrow{\mathrm{R}}_{B}^{0}-\overrightarrow{\mathrm{R}}_{A}^{0}$ & Relative coordinate of the nuclei & $\mu=\frac{M_{A} M_{B}}{M_{A}+M_{B}}$ & Reduced mass of the nuclei \\
\hline $\overrightarrow{\mathrm{r}}_{i}=\overrightarrow{\mathrm{r}}_{i}^{0}-\overrightarrow{\mathrm{R}}_{\mathrm{CMN}}$ & Coord. of electron $i$ w.r.t. CMN & $m=\frac{m_{0} M_{N}}{m_{0}+M_{N}}$ & $\begin{array}{l}\text { Molecular reduced mass of the } \\
\text { electrons }\end{array}$ \\
\hline $\overrightarrow{\mathrm{r}}_{\boldsymbol{i} A}=\overrightarrow{\mathbf{r}}_{i}^{0}-\overrightarrow{\mathrm{R}}_{A}^{0}$ & Coord. of electron $i$ w.r.t. nucleus $A$ & $m_{A}=\frac{m_{0} M_{A}}{m_{0}+M_{A}}$ & $\begin{array}{l}\text { Reduced electron mass w.r.t. } \\
\text { nucleus } A\end{array}$ \\
\hline $\overrightarrow{\mathrm{r}}_{i B}=\overrightarrow{\mathrm{r}}_{i}^{0}-\overrightarrow{\mathrm{R}}_{B}^{0}$ & Coord. of electron $i$ w.r.t. nucleus $B$ & $m_{B}=\frac{m_{0} \quad M_{B}}{m_{0}+M_{B}}$ & $\begin{array}{l}\text { Reduced electron mass w.r.t. } \\
\text { nucleus } B\end{array}$ \\
\hline $\overrightarrow{\mathrm{R}}_{\mathrm{CMA}(B)}^{0}=\frac{1}{\tilde{M}_{A(B)}}\left(M_{A(B)} \overrightarrow{\mathrm{R}}_{A(B)}^{0}+m_{0} \sum_{i=1}^{Z_{A}^{\prime}(B)} \overrightarrow{\mathrm{r}}_{i}^{0}\right)$ & Coord. of center of mass of ion $A(B)$ & $\tilde{M}_{A(B)}=M_{A(B)}+Z_{A(B)}^{\prime} m_{0}$ & Mass of ion $A(B)\left(Z_{A}^{\prime}+Z_{B}^{\prime}=Z^{\prime}\right)$ \\
\hline $\begin{aligned} \overrightarrow{\mathrm{R}}_{A B} & =\overrightarrow{\mathrm{R}}_{\mathrm{CMB}}^{0}-\overrightarrow{\mathrm{R}}_{\mathrm{CMA}}^{0} \\
& =\frac{\mu}{\mu_{A B}} \overrightarrow{\mathrm{R}}+\frac{m_{0}}{\tilde{M}_{B}} \sum_{i=1}^{Z_{B}^{\prime}} \overrightarrow{\mathrm{r}}_{i}-\frac{m_{0}}{\tilde{M}_{A}} \sum_{j=1}^{Z_{A}^{\prime}} \overrightarrow{\mathrm{r}}_{j}\end{aligned}$ & Relative distance of the two ions & $\mu_{A B}=\frac{M_{A} M_{B}}{M_{T}}$ & Reduced mass of the ions \\
\hline $\overrightarrow{\mathrm{r}}_{i A}^{*}=\overrightarrow{\mathrm{r}}_{i}^{0}-\overrightarrow{\mathrm{R}}_{C M A}^{0}=\overrightarrow{\mathrm{r}}_{i}+\frac{1}{\tilde{M}_{A}}\left(\mu \overrightarrow{\mathrm{R}}-n i_{0} \sum_{\substack{j=1 \\
7^{\prime}}}^{Z_{A}^{\prime}} \overrightarrow{\mathrm{r}}_{j}\right)$ & Coord. of electron $i$ w.r.t. CMA & $\mu_{A}=\frac{m_{0}\left[M_{A}+\left(Z_{A}^{\prime}-1\right) m_{0}\right]}{\check{M}_{A}}$ & Reduced electron mass w.r.t. CMA \\
\hline $\overrightarrow{\mathrm{r}}_{i B}^{*}=\overrightarrow{\mathrm{r}}_{i}^{0}-\overrightarrow{\mathrm{R}}_{\mathrm{CMB}}^{0}=\overrightarrow{\mathrm{r}}_{i}-\frac{1}{\tilde{M}_{B}}\left(\mu \overrightarrow{\mathrm{R}}+m_{0} \sum_{j=1}^{Z_{R}^{\prime}} \overrightarrow{\mathrm{r}}_{j}\right)$ & Coord. of electron $i$ w.r.t. CMB & $\mu_{B}=\frac{m_{0}\left[M_{B}+\left(Z_{B}^{\prime}-1\right) m_{0}\right]}{M_{B}}$ & Reduced electron mass w.r.t. $\mathrm{CM} B$ \\
\hline & & $\lambda=\frac{M_{A}-M_{B}}{M_{A}+M_{B}} \geqslant 0$ & Nuclear mass asymmetry \\
\hline
\end{tabular}




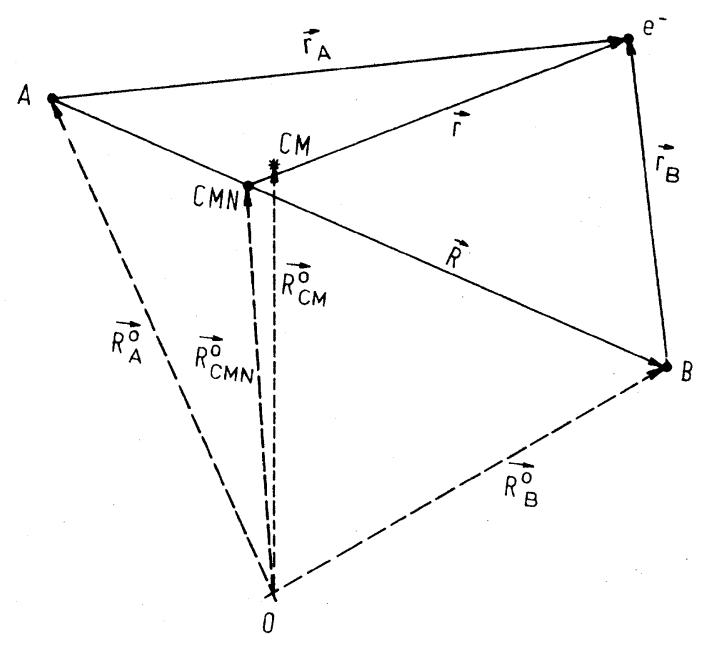

FIG. 1. Some of the coordinates used in this paper.

$$
\begin{aligned}
& \frac{1+\lambda}{2}=\frac{M_{A}}{M_{N}}=\frac{\mu}{M_{B}}=f_{B} \frac{f_{B}+\lambda}{2}, \\
& \frac{1-\lambda}{2}=\frac{M_{B}}{M_{N}}=\frac{\mu}{M_{A}}=f_{A} \frac{f_{A}+\lambda}{2}, \\
& \frac{1-\lambda^{2}}{4}=\frac{\mu}{M_{N}} .
\end{aligned}
$$

\section{A. The total Hamiltonian}

Nonrelativistically, the total kinetic energy is given as a sum of the kinetic energy of the center of mass and the energy of the relative motion:

$$
T=T_{\mathrm{CM}}+T_{\mathrm{rel}}=\frac{\overrightarrow{\mathrm{p}}^{2}}{2 \mu}+\sum_{i=1}^{Z^{\prime}} \frac{\overrightarrow{\mathrm{p}}_{i}^{2}}{2 m_{0}}+\frac{1}{2 M_{N}}\left(\sum_{i=1}^{z^{\prime}} \overrightarrow{\mathrm{P}}_{i}\right)^{2} \text {. }
$$

Here $\vec{P}$ and $\vec{p}_{i}$ are the momentum operators canonically conjugate to the coordinates $\vec{R}$ and $\vec{r}_{i}$.

We now want to find out how (2.1) has to be substituted if the electrons are to be described as relativistic spinor fields, whereas the nuclear relative motion remains nonrelativistic. Let us choose as inertial system the CM system $\left(\overrightarrow{\mathbf{P}}_{\mathrm{CM}}^{0}\right.$ $=0$ ) and consider the classical expression for the total energy without interaction
TABLE II. Order of magnitude of the expansion parameters in Eqs. (2.2) and (2.3).

\begin{tabular}{lcc}
\hline \hline & Proton-proton & $\mathrm{Pb}-\mathrm{Pb}$ \\
\hline$\frac{P^{2} / 2 \mu}{M_{N} c^{2}}$ & $\sim 5 \times 10^{-4}$ & $\sim 10^{-3}$ \\
$\frac{m_{0}}{M_{N}}$ & $\sim 3 \times 10^{-4}$ & $\sim 10^{-6}$ \\
\hline \hline & \\
$H_{0}=\left[M_{A}^{2} c^{4}+\left(P_{A}^{0}\right)^{2} c^{2}\right]^{1 / 2}+\left[M_{B}^{2} c^{4}+\left(P_{B}^{0}\right)^{2} c^{2}\right]^{1 / 2}$ \\
$+\sum_{i=1}^{Z^{\prime}}\left[m_{0}^{2} c^{4}+\left(p_{i}^{0}\right)^{2} c^{2}\right]^{1 / 2}$,
\end{tabular}

where $\overrightarrow{\mathrm{P}}_{A}^{0}$ is the canonically conjugate momentum of $\vec{R}_{A}^{0}$, etc. Expressing this in terms of $\overrightarrow{\mathbf{P}}_{C M}^{0}=0, \overrightarrow{\mathbf{P}}$ and $\overrightarrow{\mathrm{p}}_{i}$ and expanding with respect to the small entities $\left(P^{2} / 2 \mu\right) / M_{N} c^{2}$ and $m_{0} / M_{N}$, we obtain

$$
\begin{aligned}
H_{0} & =M_{N} c^{2}+\frac{P^{2}}{2 \mu}+\sum_{i=1}^{z^{\prime}}\left(m_{0}^{2} c^{4}+p_{i}^{2} c^{2}\right)^{1 / 2}+\frac{m_{0}}{M_{N}} \frac{\left(\sum^{z^{\prime}} \overrightarrow{\mathrm{p}}_{i}\right)^{2}}{2 m_{0}} \\
= & H_{\mathrm{kin}}^{\mathrm{nuc}} \\
& +H_{\mathrm{kin}}^{\mathrm{el}} .
\end{aligned}
$$

The smallness of the expansion parameters can be visualized in Table II for collisions at the Coulomb barrier. One clearly sees that for heavy systems higher-order corrections in $m_{0} / M_{N}$ can be neglected if one does not take into account simultaneously relativistic corrections to the nuclear relative motion.

Now we may quantize (2.3). Describing the nuclear relative motion by a Schrödinger wave function and the electrons by Dirac spinors, we may write down

$$
\begin{aligned}
H_{0}-M_{N} c^{2}= & \frac{P^{2}}{2 \mu}+\sum_{i=1}^{z^{\prime}}\left(\vec{\alpha}^{(i)} \cdot \overrightarrow{\mathrm{p}}_{i} c+\beta^{(i)} m_{0} c^{2}\right) \\
& +\frac{1}{2 M_{N}}\left(\sum_{i=1}^{z^{\prime}} \overrightarrow{\mathrm{p}}_{i}\right)^{2},
\end{aligned}
$$

where $\alpha^{(i)}, \beta^{(i)}$ act on electron $i$. Adding the electrostatic interaction (magnetic field effects will be included later), the Hamiltonian becomes

$$
\begin{aligned}
H & =H_{0}-M_{N} c^{2}+V=\frac{P^{2}}{2 \mu}+V^{A B}(R) \\
& \left.+\sum_{i=1}^{Z^{\prime}}\left[\overrightarrow{\boldsymbol{\alpha}}^{(i)} \overrightarrow{\mathrm{p}}_{i} c+\beta^{(i)} m_{0} c^{2}+V^{e A}\left(\overrightarrow{\mathrm{r}}_{i}, \overrightarrow{\mathrm{R}}\right)+V^{e B}\left(\overrightarrow{\mathrm{r}}_{i}, \overrightarrow{\mathrm{R}}\right)\right]+\frac{1}{2 M_{N}}\left(\sum_{i=1}^{Z^{\prime}} \overrightarrow{\mathrm{P}}_{i}\right)^{2}+\frac{1}{2} \sum_{i \neq j}^{Z^{\prime}} V^{e e}\left(\left|\overrightarrow{\mathrm{r}}_{i}-\overrightarrow{\mathrm{r}}_{j}\right|\right)_{i}\right] \\
& \equiv \frac{P^{2}}{2 \mu}+V^{A B}(R)+H_{\mathbf{m} 01}^{e 1}(\overrightarrow{\mathrm{r}}, \overrightarrow{\mathrm{R}}) .
\end{aligned}
$$


[Equation (2.5) results from a first-order $S$ matrix expansion-back reaction of the interaction on the kinetic energy via effective masses for the interacting particles, etc., and the resulting problems in defining the (CM) system are neglected. Only under this assumption is quantization as in (2.4) justified.] $H_{\mathrm{mol}}^{\mathrm{el}}(\overrightarrow{\mathrm{r}}, \overrightarrow{\mathrm{R}})$ is the relativistic molecular Hamiltonian for the electrons $\left(\overrightarrow{\mathrm{r}}\right.$ denotes the set $\left\{\overrightarrow{\mathrm{r}}_{i}\right\}$ ). It contains as a correction to the two-center-Dirac Hamiltonian

$$
\left.\sum_{i=1}^{z^{\prime}} \vec{\alpha}^{(i)} \cdot \overrightarrow{\mathrm{p}}_{i} c+\beta^{(i)} m_{0} c^{2}+V^{e A}\left(\overrightarrow{\mathrm{r}}_{i}, \overrightarrow{\mathrm{R}}\right)+V^{e B}\left(\overrightarrow{\mathrm{r}}_{i}, \overrightarrow{\mathrm{R}}\right)\right]
$$

besides the electron-electron interaction another term which takes into account that the complete electron cloud may move with respect to the CM. This term is by a factor of $m_{0} / M_{N}$ smaller than the electron kinetic energy. The nuclear relative motion is governed by the nucleus-nucleus Coulomb potential $V^{A B}(R)$.

For the purpose of later use we want to state without proof the representation of $H_{0}$ in atomic coordinates. This can be easily evaluated by using Table I and the chain rule for the momentum operators (see Ref. 21 for the same discussion for nonrelativistic electrons):

$$
H_{0}-M_{N} c^{2}=\frac{P_{A B}^{2}}{2 \mu_{A B}}+\left[\sum_{i=1}^{Z_{A}^{\prime}}\left(\vec{\alpha}^{(i)} \cdot \overrightarrow{\mathrm{p}}_{i A}^{*} c+\beta^{(i)} m_{0} c^{2}\right)-\frac{1}{2 \tilde{M}_{A}}\left(\sum_{i=1}^{Z_{A}^{\prime}} \overrightarrow{\mathrm{p}}_{i A}^{*}\right)^{2}\right]+[A-B] .
$$

\section{B. The wave function}

As the total energy $E$ is conserved, the total system (two nuclei plus $Z^{\prime}$ electrons) is described by an eigenfunction of $H$ :

$$
H(\overrightarrow{\mathrm{r}}, \overrightarrow{\mathrm{R}}) \Psi(\overrightarrow{\mathrm{r}}, \overrightarrow{\mathrm{R}})=E \Psi(\overrightarrow{\mathrm{r}}, \overrightarrow{\mathrm{R}}) .
$$

$\Psi(\vec{r}, \vec{R})$ contains the electronic motion as well as the nuclear relative motion. It may be expanded with respect to a set of electron eigenstates, for example, molecular eigenstates of $H_{\mathrm{mol}}^{\mathrm{el}}(\overrightarrow{\mathrm{r}}, \overrightarrow{\mathrm{R}})$ :

$$
H_{\mathrm{mol}}^{\mathrm{el}}(\overrightarrow{\mathrm{r}}, \overrightarrow{\mathrm{R}}) \varphi_{n}(\overrightarrow{\mathrm{r}}, \overrightarrow{\mathrm{R}})=\epsilon_{n}(R) \varphi_{n}(\overrightarrow{\mathrm{r}}, \overrightarrow{\mathrm{R}}) .
$$

For asymmetric systems $(A \neq B)$ the bound states of the molecular basis for $R \rightarrow \infty$ go over into a product of wave functions localized around the two nuclei $A, B$ (Ref. 20):

$$
\varphi_{n}(\overrightarrow{\mathrm{r}}, \overrightarrow{\mathrm{R}}) \underset{R \rightarrow \infty}{\longrightarrow} \varphi_{n_{A}}\left(\overrightarrow{\mathrm{r}}_{A}^{*}\right) \varphi_{a_{B}}\left(\overrightarrow{\mathrm{r}}_{B}^{*}\right) .
$$

For symmetrical systems the $\varphi_{n}(\overrightarrow{\mathrm{r}}, \overrightarrow{\mathrm{R}})$ additionally have good parity, and a certain combination of even and odd states has the property (2.9). This property is due to the fact that for $R \rightarrow \infty$ an electron in ion $A$ no longer feels ion $B$. This is not true for continuum electrons; continuum energy eigenstates are always spread over all space and always feel the potential of both nuclei. Therefore, for continuum electrons (2.9) will not hold. We will discuss this problem in Sec. IV.

For the following let us assume asymmetric systems and that (2.9) be fulfilled. Expanding $\Psi(\vec{r}, \vec{R})$ with respect to the molecular basis $\varphi_{n}(\overrightarrow{\mathrm{r}}, \overrightarrow{\mathrm{R}})$ (perturbated-stationary-state method), we find

$$
\Psi(\overrightarrow{\mathrm{r}}, \overrightarrow{\mathrm{R}})=\sum_{n} \chi_{n}(\overrightarrow{\mathrm{R}}) \varphi_{n}(\overrightarrow{\mathrm{r}}, \overrightarrow{\mathrm{R}}),
$$

where $\chi_{n}(\vec{R})$ contains the nuclear relative motion. Projecting out the molecular states $\varphi_{n}$ would yield a set of differential equations for $\chi_{n}$ which, by semiclassical approximation, would lead to the coupled-channel equations (1.4). This method, called "perturbated-stationary-state (PSS) method," thus leads to the various long-range couplings discussed in the introduction. We can now trace the reason for that to the fact that for $R \rightarrow \infty\left(V^{A B} \rightarrow 0\right)$ the $\chi_{n}(\vec{R})$ become eigenfunctions of $P^{2} / 2 \mu$, i.e., plane waves with a good momentum $\mathrm{P}$ :

$$
\chi_{n} \underset{R \rightarrow \infty}{\longrightarrow} e^{(i / \hbar) \overrightarrow{\mathrm{P}} \cdot \overrightarrow{\mathrm{R}}}
$$

Physically, however, we expect the asymptotic relative motion to be that of two ions (not nuclei) moving apart, described by eigenfunctions of the momentum $\overrightarrow{\mathrm{P}}_{A B}$ canonically conjugate to the interatomic distance $\overrightarrow{\mathrm{R}}_{A B}$ :

$$
\chi_{n} \underset{R \rightarrow \infty}{\longrightarrow} e^{(i / \hbar) \vec{P}_{A B} \cdot \overrightarrow{\mathrm{R}}_{A B}} .
$$

Obviously $\vec{R}$ is not the asymptotically correct scattering coordinate. Therefore, it is convenient to use another scattering coordinate becoming $\vec{R}_{A B}$ as $R \rightarrow \infty$. This idea is due to Mittleman and Tai. ${ }^{19}$ They changed from the molecular basis $\varphi_{n}(\overrightarrow{\mathrm{r}}, \overrightarrow{\mathrm{R}})$ to a new basis $\varphi_{n}\left(\vec{r}, \vec{R}^{*}\right)$ in such a way that in the expansion

$$
\Psi(\overrightarrow{\mathrm{r}}, \overrightarrow{\mathrm{R}})=\sum_{n} \tilde{\chi}_{n}\left(\mathrm{R}^{*}\right) \varphi_{n}\left(\overrightarrow{\mathrm{r}}, \overrightarrow{\mathrm{R}}^{*}\right)
$$

the $\bar{\chi}_{n}$ behaved asymptotically correctly:

$$
\tilde{\chi}_{n}\left(\overrightarrow{\mathrm{R}}^{*}\right) \underset{R \rightarrow \infty}{\longrightarrow} e^{(i / \hbar) \overrightarrow{\mathrm{P}}_{A B} \cdot \overrightarrow{\mathrm{R}}_{A B}}
$$


Mittleman and Tai expressed all wave functions through $\overrightarrow{\mathrm{R}} *=\overrightarrow{\mathrm{R}}^{*}(\overrightarrow{\mathrm{r}}, \overrightarrow{\mathrm{R}})$ and thus had for every $\overrightarrow{\mathrm{R}}$ and for every electron configuration $\left\{\vec{r}_{i}\right\}$ a new "molecular" basis $\varphi_{n}(\overrightarrow{\mathrm{r}}, \mathrm{R})$. We, however, will explicitly write out the coordinate transformation $\vec{R} \rightarrow \vec{R} *$ and then be able to continue to use the old set of coordinates $\left\{\vec{R}, \vec{r}_{i}\right\}$ and hence the twocenter basis $\varphi_{n}(\vec{r}, \vec{R})$. Using the new scattering coordinate $\vec{R}^{*}$ will thus result in additional easily calculable coupling matrix elements between the molecular states $\varphi_{n}(\overrightarrow{\mathrm{r}}, \overrightarrow{\mathrm{R}})$.

$$
\begin{aligned}
& \text { We now set } \\
& \vec{R} *=\vec{R}+\vec{x},
\end{aligned}
$$

and thus may write

$$
\Psi(\overrightarrow{\mathrm{r}}, \overrightarrow{\mathrm{R}})=\sum_{n} \hat{T}(\overrightarrow{\mathrm{X}})\left(\tilde{\chi}_{n}(\overrightarrow{\mathrm{R}}) \varphi_{n}(\overrightarrow{\mathrm{r}}, \overrightarrow{\mathrm{R}}),\right.
$$

where $\hat{T}(\vec{X})$ is a translation operator

$$
\hat{T}(\vec{\chi}) f(\overrightarrow{\mathrm{R}})=f(\overrightarrow{\mathrm{R}}+\overrightarrow{\mathrm{X}}) \text {. }
$$

For constant $\vec{X}$ we have the representation

$$
\hat{T}(\overrightarrow{\mathrm{X}})=e^{\overrightarrow{\mathrm{X}} \cdot \vec{\nabla}_{R}}=e^{(i / h) \overrightarrow{\mathrm{X}} 6 \overrightarrow{\mathrm{P}}} .
$$

In our case," however, $\vec{X}$ will depend upon $\vec{R}$ (see below), and we have

$$
\hat{T}(\overrightarrow{\mathrm{X}})=1+\frac{i}{\hbar} X_{i} P_{i}+\frac{1}{2}\left(\frac{i}{\hbar}\right)^{2} X_{j} X_{i} P_{i} P_{j}+\cdots
$$

(summation over double indices is understood). The adjoint operator $\hat{T}^{\dagger}(\vec{X})$ is obtained by partial integration:

$$
\hat{T}^{\dagger}(\overrightarrow{\mathrm{X}})=1-\frac{i}{\hbar} P_{i} X_{i}+\frac{1}{2}\left(\frac{-i}{\hbar}\right)^{2} P_{i} P_{j} X_{i} X_{j}+\cdots
$$

Since the momentum operator $\overrightarrow{\mathrm{P}}$ affects $\overrightarrow{\mathrm{X}}, \hat{T}$ is not unitary, i.e., $\hat{T}^{\dagger}(\overrightarrow{\mathrm{X}}) \hat{T}(\overrightarrow{\mathrm{X}}) \neq 1$.

The reason for this nonunitarity is that we continue to express everything through $(\vec{R}, \vec{r})$ and not through $\left(\overrightarrow{\mathrm{R}}^{*}, \overrightarrow{\mathrm{r}}\right)$. In particular, the volume element in the scalar product $d^{3} R d^{3} r_{1} \ldots d^{3} r_{Z}$, is kept unchanged. $\hat{T}^{\dagger}(\overrightarrow{\mathrm{X}}) \hat{T}(\overrightarrow{\mathrm{X}})$ is nothing but the Jacobian $\left\|\partial R / \partial R^{*}\right\|$, which we have to take into account explicitly. We could avoid this by introducing a new scalar product $d^{3} R^{*}$ $\times d^{3} r_{1} \ldots d^{3} r_{z^{\prime}}$, but then we would have to redefine all differential operators in the Hamiltonian in order to again obtain Hermiticity.

Another consequence of $\hat{T}^{\dagger} \hat{T} \neq 1$ is that the new coefficients $\tilde{\chi}_{n}$ differ slightly from $\chi_{n}$ as functions. From the normalization condition for $\Psi$,

$$
\int \Psi^{\mathrm{t}}(\overrightarrow{\mathrm{r}}, \overrightarrow{\mathrm{R}}) \Psi(\overrightarrow{\mathrm{r}}, \overrightarrow{\mathrm{R}}) d^{3} R d^{3} \boldsymbol{r}_{1} \ldots d^{3} \boldsymbol{r}_{\boldsymbol{z}^{\circ}}=C,
$$

we get, using (2.10),

$$
\begin{aligned}
\sum_{m, n} \int \tilde{\chi}_{n}^{*}(\overrightarrow{\mathrm{R}}) \varphi_{n}^{\dagger}(\overrightarrow{\mathrm{r}}, \overrightarrow{\mathrm{R}}) \chi_{m}(\overrightarrow{\mathrm{R}}) \\
\quad \times \varphi_{m}(\overrightarrow{\mathrm{r}}, \overrightarrow{\mathrm{R}}) d^{3} R d^{3} r_{1} \ldots d^{3} r_{\mathbf{Z}^{\prime}}=C,
\end{aligned}
$$

whereas (2.16) yields

$$
\begin{aligned}
\sum_{m=n} \int \tilde{\chi}_{n}^{*}(\overrightarrow{\mathrm{R}}) \varphi_{n}^{\dagger}(\overrightarrow{\mathrm{r}}, \overrightarrow{\mathrm{R}}) \hat{T}^{\dagger}(\overrightarrow{\mathrm{X}}) \hat{T}(\overrightarrow{\mathrm{X}}) \tilde{\chi}_{m}(\overrightarrow{\mathrm{R}}) \\
\times \varphi_{m}(\mathrm{r}, \mathrm{R}) d^{3} R d^{3} \boldsymbol{r}_{1} \ldots d^{3} \boldsymbol{r}_{\mathbf{z}^{\prime}}=C
\end{aligned}
$$

In lowest order $(m / \mu) \hat{T}^{\dagger} \hat{T}$ is a real number (see Appendix A); hence, comparing (2.22) and (2.23), it follows that

$$
\begin{aligned}
\tilde{\chi}_{n} & =\left(\hat{T}^{\dagger} \hat{T}\right)^{-1 / 2} \chi_{n} \\
& \cong\left(1-\frac{3}{16} \frac{m}{\mu} \sum_{i=1}^{Z^{\prime}}\left[f\left(\overrightarrow{\mathrm{r}}_{i}, \overrightarrow{\mathrm{R}}\right)+\lambda\right]^{2}\right) \chi_{n} .
\end{aligned}
$$

Equations (2.21)-(2.24) guarantee that the norm is conserved under the transformation in spite of $\hat{T}$ being nonunitary. Moreover, we recognize as the proper transformation operator the unitary combination (see Appendix A) $\left(\hat{T}^{\dagger} \hat{T}\right)^{-1 / 2} \hat{T}$. Hence the completeness of the expansion of the total wave function with respect to the $\varphi_{n}(\vec{r}, \vec{R} *)$ is no problem.

The problem of $\hat{T}$ not being unitary in case $\vec{X}$ $=\overrightarrow{\mathrm{X}}(\overrightarrow{\mathrm{R}})$ was not correctly recognized in Ref. 20 . Thorson and Delos used the representation (2.18) and for $\hat{T}^{t}(\overrightarrow{\mathrm{X}})$ they wrote $e^{(-i / \hbar) \hat{\mathrm{X}} \cdot \overrightarrow{\mathrm{P}}}, \overrightarrow{\mathrm{P}}$ being the momentum operator. Then they had $\hat{T}^{\dagger} \hat{T}=1$, but their transformed Hamiltonian was not Hermitian. Hermiticity they established afterwards by symmetrizing several terms, thereby obtaining a slightly different result than we do. Our result will be fully consistent in order $m / \mu$.

We now are going to calculate the translation vector $\overrightarrow{\mathrm{X}}$.

$$
\text { C. The translation vector } \overrightarrow{\mathrm{X}}
$$

The translation vector $\vec{X}$ is defined by two asymptotic conditions:

(i) For $R \rightarrow 0$ we want to work in the molecular picture. Hence in this limit the internuclear distance $\vec{R}$ is the correct scattering coordinate.

(ii) For $R \rightarrow \infty, \vec{R}^{*}=\overrightarrow{\mathrm{R}}+\overrightarrow{\mathrm{X}}$ should become $\overrightarrow{\mathrm{R}}_{A B}$, i.e., the interatomic distance; this scattering coordinate takes into account the asymptotic translation of the electron with the two nuclei.

For the details of the calculation of $\vec{X}$ out of these boundary conditions we refer to Ref. 20 . The result is 


$$
\overrightarrow{\mathrm{X}}=\frac{m}{\mu} \sum_{i=1}^{\mathbf{Z}^{\prime}} \overrightarrow{\mathbf{s}}_{\boldsymbol{i}}
$$

with

$$
\overrightarrow{\mathrm{s}}_{i}=\frac{f\left(\overrightarrow{\mathrm{r}}_{i}, \overrightarrow{\mathrm{R}}\right)+\lambda}{2}\left(\overrightarrow{\mathrm{r}}_{i}-\frac{f\left(\overrightarrow{\mathrm{r}}_{i}, \overrightarrow{\mathrm{R}}\right)+\lambda}{4} \overrightarrow{\mathrm{R}}\right) .
$$

Here $f\left(\overrightarrow{\mathbf{r}}_{i}, \overrightarrow{\mathrm{R}}\right)$ is a so-called "switching function" which is free up to the boundary conditions for $R \rightarrow 0$ and $R \rightarrow \infty$. Condition (i) is fulfilled by ( $\lambda$ is the mass asymmetry)

$$
\lim _{r \rightarrow \infty, R / r \rightarrow 0} f(\overrightarrow{\mathrm{r}}, \overrightarrow{\mathrm{R}})=-\lambda .
$$

Condition (ii) leads to

$$
\begin{array}{r}
\lim _{R \rightarrow \infty, r_{B} / R \rightarrow 0} f(\overrightarrow{\mathrm{r}}, \overrightarrow{\mathrm{R}})=f_{B}=+1, \\
\lim _{R \rightarrow \infty, r_{A^{\prime}}} f(\overrightarrow{\mathrm{r}}, \overrightarrow{\mathrm{R}})=f_{A}=-1 .
\end{array}
$$

Two choices for $f(\overrightarrow{\mathbf{r}}, \overrightarrow{\mathrm{R}})$ fulfilling (2.27) and (2.28) will be discussed in Sec. V.

\section{The equations of nuclear relative motion}

Inserting (2.16) into the Schrödinger equation (2.7), we obtain

$$
\sum_{n}[H(\overrightarrow{\mathrm{r}}, \overrightarrow{\mathrm{R}})-E] \hat{T}(\overrightarrow{\mathrm{X}}) \tilde{\chi}_{n}(\overrightarrow{\mathrm{R}}) \varphi_{n}(\overrightarrow{\mathrm{r}}, \overrightarrow{\mathrm{R}})=0 .
$$

Multiplying from the left by

$$
\left[\hat{T}^{\dagger}(\overrightarrow{\mathrm{X}}) \hat{T}(\overrightarrow{\mathrm{X}})\right]^{1 / 2} \hat{T}^{-1}(\overrightarrow{\mathrm{X}}),
$$

using (2.24), and forming the scalar product with $\varphi_{m}^{\dagger}(\overrightarrow{\mathrm{r}}, \overrightarrow{\mathrm{R}})$ yields

$$
\sum_{n}\left\langle m\left|H^{\prime}-E\right| n\right\rangle \chi_{n} \equiv \sum_{n} \int d^{3} \gamma_{1} \ldots d^{3} \gamma_{z^{\prime}} \varphi_{m}^{\dagger}(\overrightarrow{\mathrm{r}}, \overrightarrow{\mathrm{R}})\left[\left(\hat{T}^{\dagger} \hat{T}\right)^{1 / 2} \hat{T}^{-1} H \hat{T}\left(\hat{T}^{\dagger} \hat{T}\right)^{-1 / 2}-E\right] \varphi_{n}(\overrightarrow{\mathrm{r}}, \overrightarrow{\mathrm{R}}) \chi_{n}(\overrightarrow{\mathrm{R}})=0
$$

We recognize that the coordinate transformation $\vec{R} \rightarrow \vec{R}^{*}$ can be rewritten as a unitary transformation on $H(\overrightarrow{\mathrm{r}}, \overrightarrow{\mathrm{R}})$ :

$$
H^{\prime}(\overrightarrow{\mathrm{r}}, \overrightarrow{\mathrm{R}})=\left[\hat{T}^{\dagger}(\overrightarrow{\mathrm{X}}) \hat{T}(\overrightarrow{\mathrm{X}})\right]^{1 / 2} \hat{T}^{-1}(\overrightarrow{\mathrm{X}}) H(\overrightarrow{\mathrm{r}}, \overrightarrow{\mathrm{R}}) \hat{T}(\overrightarrow{\mathrm{X}})\left[\hat{T}^{\dagger}(\overrightarrow{\mathrm{X}}) \hat{T}(\overrightarrow{\mathrm{X}})\right]^{-1 / 2}
$$

Hence (2.30) has the same structure as in the PSS theory; however, $H$ is substituted by $H^{\prime}$. $H^{\prime}$ cannot be given in closed form. However, as $\hat{T}$ is generated by the small vector $\overrightarrow{\mathrm{X}}=(m / \mu) \sum \mathbf{i}_{i=1}^{\mathbf{z}^{\prime}} \overrightarrow{\mathrm{s}}_{i}$, we may expand $H^{\prime}(\overrightarrow{\mathrm{r}}, \overrightarrow{\mathrm{R}})$ with respect to $m / \mu$. As we saw in Sec. II.A, the operator $H$ is only consistent in lowest-order $m / \mu$ because we used the nonrelativistic approximation for the nuclear relative motion. Hence, in order to stay consistent, we should drop all terms $\sim(m / \mu)^{n}, n \geqslant 2$ in the expansion. In addition, we shall assume that the switching function is so smooth that we may safely drop terms $\sim(m / \mu) \operatorname{grad}_{r} f$ and $\sim(m / \mu) \operatorname{grad}_{R} f$. This considerably simplifies the expression for $H^{\prime}$. [For a sudden change of the basis (e.g., $f$ jumps from 0 to \pm 1 at a certain distance), there may occur errors on the order of $m / \mu$. We will see, however, that as long as $f$ is smooth as a function of $\vec{r}$, the essential corrections $\vec{A}_{m n}$ are not affected.]

For the details of the calculation we refer to Appendix A. For the transformed Hamiltonian one obtains in order $m / \mu$ :

$H^{\prime}(\overrightarrow{\mathrm{r}}, \overrightarrow{\mathrm{R}})=\frac{P^{2}}{2 \mu}+V^{A B}(R)+H_{\mathrm{mo} 1}^{\mathrm{el}}(\overrightarrow{\mathrm{r}}, \overrightarrow{\mathrm{R}})+\Delta+\frac{1}{2 \mu}\{\overrightarrow{\mathrm{A}}, \overrightarrow{\mathrm{P}}\}$,

where $\Delta$ is the difference between transformed and old potential:

$$
\begin{aligned}
\Delta= & V^{A B}(|\overrightarrow{\mathrm{R}}-\overrightarrow{\mathrm{X}}|)-V^{A B}(R) \\
& +\sum_{i=1}^{Z^{\prime}}\left[V^{e A}\left(\overrightarrow{\mathrm{r}}_{i}, \overrightarrow{\mathrm{R}}-\overrightarrow{\mathrm{X}}\right)-V^{e A}\left(\overrightarrow{\mathrm{r}}_{i}, \overrightarrow{\mathrm{R}}\right)\right. \\
& \left.+V^{e B}\left(\overrightarrow{\mathrm{r}}_{i}, \overrightarrow{\mathrm{R}}-\overrightarrow{\mathrm{X}}\right)-V^{e B}\left(\overrightarrow{\mathrm{r}}_{i}, \overrightarrow{\mathrm{R}}\right)\right] .
\end{aligned}
$$

The operator $\vec{A}$ is defined by

$$
\begin{aligned}
\overrightarrow{\mathrm{A}} & =\frac{i}{\hbar} \mu\left[H_{\mathrm{mol} 1}^{\mathrm{e}}(\overrightarrow{\mathrm{r}}, \overrightarrow{\mathrm{R}}), \overrightarrow{\mathrm{X}}\right] \\
& =m \sum_{l=1}^{Z^{\prime}} \frac{f(\overrightarrow{\mathrm{r}}, \overrightarrow{\mathrm{R}})+\lambda}{2} \vec{\alpha}^{(l)} c .
\end{aligned}
$$

From (2.30) we thus find

$$
\sum_{n}\left\langle m\left|\frac{P^{2}}{2 \mu}+V^{A B}(R)+H_{\mathrm{mol}}^{\mathrm{el}}(\overrightarrow{\mathrm{r}}, \overrightarrow{\mathrm{R}})+\Delta(\overrightarrow{\mathrm{r}}, \overrightarrow{\mathrm{R}})+\frac{1}{2 \mu}(\overrightarrow{\mathrm{A}} \cdot \overrightarrow{\mathrm{P}}+\overrightarrow{\mathrm{P}} \cdot \overrightarrow{\mathrm{A}})-E\right| n\right\rangle \chi_{n}(\overrightarrow{\mathrm{R}})=0,
$$


which may easily be evaluated to

$$
\sum_{n}\left[\frac{1}{2 \mu}[\overrightarrow{\mathrm{P}} \underline{1}+\underline{\overrightarrow{\mathrm{P}}}+\underline{\overrightarrow{\mathrm{A}}}]_{m n}^{2}+\left[V^{A B}(R)+\epsilon_{n}(R)-E\right] \delta_{m n}+\left(\Delta-\frac{1}{2 \mu} \underline{\overrightarrow{\mathrm{A}}} \cdot \underline{\overrightarrow{\mathrm{A}}}\right){ }_{m n}\right] \chi_{n}(\overrightarrow{\mathrm{R}})=0 .
$$

Here $(\underline{1})_{m n}=\delta_{m n}=\langle m \mid n\rangle$ is the unit matrix;

$$
(\overrightarrow{\mathrm{P}})_{m n}=\langle m|\overrightarrow{\mathrm{P}}| n\rangle
$$

are the matrix elements of the momentum operator $\overrightarrow{\mathrm{P}}$ between the molecular basis states (which turns out to be just the usual dynamical coupling);

$$
(\underline{\vec{A}})_{m n}=\langle m|\vec{A}| n\rangle
$$

will become the corrections of the dynamical coupling due to the electron translation; and

$$
(\Delta)_{m n} \equiv\langle m|\Delta(\overrightarrow{\mathrm{r}}, \overrightarrow{\mathrm{R}})| n\rangle \text {. }
$$

Comparing the result (2.36) with the corresponding equation from the PSS theory,

$$
\begin{aligned}
\sum_{n}( & \frac{1}{2 \mu}(\overrightarrow{\mathrm{P}} \cdot \underline{1}+\underline{\overrightarrow{\mathrm{P}}})_{m n}^{2} \\
& \left.+\left(V^{A B}+\epsilon_{n}-E\right) \delta_{m n}\right) \chi_{n}(\overrightarrow{\mathrm{R}})=0,
\end{aligned}
$$

we will see that the really essential difference is the substitution $\underline{\overrightarrow{\mathrm{P}}} \rightarrow \underline{\overrightarrow{\mathrm{P}}}+\underline{\overrightarrow{\mathrm{A}}}$. All other corrections are smaller by an order $m / \mu$. The important point is that whereas $\underline{\overrightarrow{\mathrm{p}}}$ does not vanish for $R \rightarrow \infty$, $\underline{\vec{P}}+\underline{\vec{A}}$ does:

$$
\lim _{R \rightarrow \infty}\left(\overrightarrow{\mathrm{P}}_{m n}+\overrightarrow{\mathrm{A}}_{m n}\right)=0
$$

Hence the correction matrix elements due to electron translation asymptotically cancel the spurious dynamical couplings.

The proof of (2.41) is not difficult in the case of non relativistic electronic motion,$^{20}$ using that one of the two states involved obeys (2.9). We now want to sketch the slightly more involved reasoning for the case of relativistic electrons, which also will be of use later in Sec. IV.

Let us first express the molecular Hamiltonian $H_{\mathrm{mol}}^{\mathrm{el}}(\overrightarrow{\mathrm{r}}, \overrightarrow{\mathrm{R}})$ in terms of atomic momenta $\overrightarrow{\mathrm{P}}_{A B}, \vec{p}_{i A}^{*}$, $\vec{p}_{i_{B}}^{*}$. (This is achieved by using Table I for the coordinates and applying the chain rule for the differential operators $\vec{p}_{i A}^{*}$, etc.) We obtain

$$
\begin{aligned}
& H_{\mathrm{mol}}^{\mathrm{el}}(\overrightarrow{\mathrm{r}}, \overrightarrow{\mathrm{R}})=\left[\sum_{i=1}^{z^{\prime}}\left[\overrightarrow{\mathrm{a}}^{(i)} \cdot \overrightarrow{\mathrm{p}}_{i A}^{*} c+\beta^{(i)} m_{0} c^{2}+V^{e A}\left(\overrightarrow{\mathrm{r}}_{i A}\right)\right]+\frac{1}{2} \sum_{i+j}^{Z_{A}^{\prime}} V^{e e}\left(\left|\overrightarrow{\mathrm{r}}_{i}-\overrightarrow{\mathrm{r}}_{j}\right|\right)-\frac{1}{2 \tilde{M}_{A}}\left(\sum_{i=1}^{Z_{A}^{\prime}} \vec{p}_{i A}^{*}\right)^{2}\right]+[A \rightarrow B] \\
& +\left\{\left[\sum_{i=1}^{Z^{\prime} A}\left(-\sum_{j=1}^{Z_{A}^{\prime}} \frac{m_{0}}{\tilde{M}_{A}} \alpha^{(i)} \cdot \overrightarrow{\mathrm{p}}_{j A}^{*} c+V^{e B}\left(\overrightarrow{\mathrm{r}}_{i_{B}}\right)+\frac{1}{2} \sum_{j=1}^{Z_{B}^{\prime}} V^{e e}\left(\left|\overrightarrow{\mathrm{r}}_{i}-\overrightarrow{\mathrm{r}}_{j}\right|\right)\right)+\frac{1}{2 \tilde{M}_{A}}\left(\sum_{i}^{Z_{A}^{\prime}} \overrightarrow{\mathrm{p}}_{i A}^{*}\right)^{2}\right]+[A \hookrightarrow B]\right. \\
& +\overrightarrow{\mathrm{P}}_{A B} \cdot\left(\frac{m_{0}}{\tilde{M}_{B}} \sum_{i=1}^{z_{B}^{\prime}} \vec{\alpha}^{(i)} c-\frac{m_{0}}{\tilde{M}_{A}} \sum_{i=1}^{z_{A}^{\prime}} \vec{\alpha}^{(i)} c\right) \\
& \left.+\frac{1}{2 M_{N}}\left[\frac{M_{A}}{\tilde{M}_{A}} \sum^{z_{A}^{\prime}} \overrightarrow{\mathrm{p}}_{i A}^{*}+\frac{M_{B}}{\bar{M}_{B}} \sum^{Z_{B}^{\prime}} \overrightarrow{\mathrm{p}}_{i B}^{*}+\left(\frac{Z_{B}^{\prime} m_{0}}{\bar{M}_{B}}-\frac{Z_{A}^{\prime} m_{0}}{\tilde{M}_{A}}\right) \overrightarrow{\mathrm{P}}_{A B}\right]^{2}\right\} \\
& \equiv H_{\text {at om }}^{\mathrm{el}}+\left\{H_{\text {corr }}\right\}
\end{aligned}
$$

$H_{\text {at om }}^{\text {el }}$ is just the atomic Hamiltonian [see (2.6)] for the case where the molecule dissociates into two ions with $Z_{A}^{\prime}$ and $Z_{B}^{\prime}$ electrons. If we neglect the small corrections due to the fact that $V^{e A}$ depends on $\overrightarrow{\mathrm{r}}_{i A}$ (distance to nucleus $A$ ) instead of $\vec{r}_{i A}^{*}$ (distance to the center of mass of ion $A$ ), $H_{\text {mol }}^{e l}$ in the asymptotic channel contains [up to order $(m / \mu)^{2}$ ] only one correction which depends on the atomic distance $\overrightarrow{\mathrm{R}}_{A B}$ :

$$
\overrightarrow{\mathbf{P}}_{A B}\left(\frac{m_{0}}{\tilde{M}_{B}} \sum_{i=1}^{z_{\dot{R}}^{\prime}} \vec{\alpha}^{(i)} c-\frac{m_{0}}{\tilde{M}_{A}} \sum_{i=1}^{z_{\dot{A}}} \vec{\alpha}^{(i)} c\right) \text {. }
$$

[This term is the relativistic analog to the recoil term, consideredin Refs.22(a) and 22(b) which occurs in a treatment of ion scattering in an atomic basis and is given in a somewhat different form in Ref. 23.] Comparing this to $(1 / 2 \mu)\{\vec{A}, \vec{P}\}$ we easily see that both terms asymptotically yield the same $\overrightarrow{\mathrm{P}}_{A B}$ coupling: 


$$
\begin{aligned}
\frac{1}{2 \mu} & \{\overrightarrow{\mathrm{A}}, \overrightarrow{\mathrm{P}}\} \underset{R \rightarrow \infty}{\longrightarrow} \frac{m}{\mu} \sum_{i=1}^{z^{\prime}} \frac{f_{l}+\lambda}{2} \vec{\alpha}^{(l)} \cdot \overrightarrow{\mathrm{P}} c \\
& =\frac{m}{\mu} \sum_{i=1}^{Z^{\prime}} f_{l} \frac{\mu}{M_{l}} \vec{\alpha}^{(l)} c \frac{\mu}{\mu_{A B}} \overrightarrow{\mathrm{P}}_{A B}+\frac{m}{\mu} \sum_{l=1}^{Z^{\prime}} f_{l} \frac{\mu}{M_{l}} \vec{\alpha}^{(l)} c\left(\frac{\mu}{\bar{M}_{A}} \sum_{i=1}^{Z_{A}^{\prime}} \overrightarrow{\mathrm{p}}_{i A}^{*}-\frac{\mu}{\tilde{M}_{B}} \sum_{i=1}^{Z_{B}^{\prime}} \overrightarrow{\mathrm{p}}_{i B}^{*}\right) \\
& =\overrightarrow{\mathrm{P}}_{A B}\left(\frac{m_{0}}{\tilde{M}_{B}} \sum_{i}^{z_{B}^{\prime}} \vec{\alpha}^{(i)} c-\frac{m_{0}}{\tilde{M}_{A}} \sum_{i}^{z_{A}^{\prime}} \vec{\alpha}^{(i)} c\right)-\frac{m_{0}}{\tilde{M}_{A}} \sum_{i, j}^{z_{A}^{\prime}} \vec{\alpha}^{(i)} \cdot \overrightarrow{\mathrm{p}}_{j A}^{*} c-\frac{m_{0}}{\tilde{M}_{B}} \sum_{i, j}^{z_{B}^{\prime}} \vec{\alpha}^{(i)} \cdot \overrightarrow{\mathrm{p}}_{j B}^{*} c+O\left(\frac{m^{2}}{\mu^{2}}\right) .
\end{aligned}
$$

Indeed, $(1 / 2 \mu)\{\overrightarrow{\mathrm{A}}, \overrightarrow{\mathrm{P}}\}$ yields all three underlined terms of (2.42). Thus we have shown that

$$
H_{\mathrm{at} \mathrm{om}}^{\mathrm{el}}+\frac{1}{2 \mu}\{\overrightarrow{\mathrm{A}}, \overrightarrow{\mathrm{P}}\}+V_{\mathrm{mol}}^{\mathrm{el}}-V_{\mathrm{at} \mathrm{om}}^{\mathrm{el}} \underset{R \rightarrow \infty}{\longrightarrow} H_{\mathrm{mol}}^{\mathrm{el}}+O(m / \mu) \text {. }
$$

where $V_{\text {mol }}^{\mathrm{el}}-V_{\mathrm{atom}}^{\mathrm{el}}$ is the interaction of the electrons in $A$ with nucleus $B$, and vice versa.

Equivalently,

$$
H=\frac{P^{2}}{2 \mu}+V^{A B}(R)+H_{\mathrm{mol}}^{\mathrm{el}} \underset{R \rightarrow \infty}{\longrightarrow} \frac{P^{2}}{2 \mu}+V^{A B}(R)+H_{\mathrm{at} \mathrm{om}}^{\mathrm{el}}+\frac{1}{2 \mu}\{\overrightarrow{\mathrm{A}}, \overrightarrow{\mathrm{P}}\}+V_{\mathrm{mol}}^{\mathrm{el}}-V_{\mathrm{at} \mathrm{om}}^{\mathrm{el}}+O\left(\frac{m}{\mu}\right) .
$$

Comparing this with the representation of $H$ in atomic coordinates [see (2.6)],

$H=\frac{P_{A B}^{2}}{2 \mu_{A B}}+V^{A B}(R)+H_{\mathrm{atom}}^{\mathrm{el}}+V_{\mathrm{mol}}^{\mathrm{el}}-V_{\mathrm{atom}}^{\mathrm{el}}$

we thus have proved that

$$
\frac{P^{2}}{2 \mu}+\frac{1}{2 \mu}\{\overrightarrow{\mathrm{A}}, \overrightarrow{\mathrm{P}}\} \underset{R \rightarrow \infty}{\longrightarrow} \frac{P_{A B}^{2}}{2 \mu_{A B}}\left[1+O\left(\frac{m}{\mu}\right)\right]
$$

For bound states with the property (2.9), however, $\overrightarrow{\mathrm{P}}_{A B}$ does not act on the electron wave function for $R \rightarrow \infty$, and thus the spurious long-range dynamical couplings vanish, as they should according to the idea behind the coordinate transformation $\vec{R}-\vec{R}^{*}$.

Once again we want to stress that this is not true for continuum states. In that case the interaction $V_{\mathrm{mol}}^{\mathrm{el}}-V_{\mathrm{at} \text { om }}^{\mathrm{el}}$ does not vanish for $R \rightarrow \infty$, and due to the interaction of the electrons with both centers even for $R \rightarrow \infty$, the separation property (2.9) is destroyed. Hence $\overrightarrow{\mathrm{P}}_{A B}$ also for $R \rightarrow \infty$ acts on the electron wave function, and the long-range cou- plings remain. In Sec. IV we shall show how to avoid this problem.

\section{E. Transformation to rotating coordinates}

The equations of motion (2.36) contain couplings by the relative momentum of the two nuclei $\overrightarrow{\mathbf{P}}=i \hbar \vec{\nabla}_{R} \cdot \vec{\nabla}_{R}$ means differentiation with respect to $\overrightarrow{\mathrm{R}}$ keeping the electron coordinates $\overrightarrow{\mathrm{r}}_{i}$ in the center of mass system of the nuclei CMN system) fixed. On the other hand, the electron wave functions $\varphi_{m}(\vec{r}, \vec{R})$ are most easily calculated in a coordinate system which rotates with the internuclear axis $\vec{R}$. In this system, where the electron coordinates are $\overrightarrow{\mathbf{r}}_{i}^{\prime}, \varphi_{m}\left(\overrightarrow{\mathrm{r}}^{\prime}, R\right)$ only depends upon the distance $R$ of the nuclei, and no longer on the orientation of the vector $\vec{R}$. Therefore, we will transform the equations of motion (2.36) into this rotating coordinate system. In order to do so we have to substitute all differential operators $\vec{\nabla}_{R}$, keeping $\overrightarrow{\mathrm{r}}$ fixed, into differential operators, keeping $\vec{r}^{\prime}$ fixed. The calculation is analogous to Ref. 20 and may be found in detail in Ref. 24. We make use of

$$
\left.\left(-i \hbar \vec{\nabla}_{R}\right)\right|_{\overrightarrow{\mathbf{r}}}=\left(-\left.i \hbar \frac{\partial}{\partial R}\right|_{\overrightarrow{\mathbf{r}}^{\prime}} ;-\left.i \hbar \frac{1}{R} \frac{\partial}{\partial \theta}\right|_{\overrightarrow{\mathbf{r}}^{\prime}}-\frac{1}{R} J_{y^{\prime}} ;-\left.i \hbar \frac{1}{R \sin \theta} \frac{\partial}{\partial \varphi}\right|_{\overrightarrow{\mathbf{r}}^{\prime}}+\frac{1}{R} J_{x^{\prime}}-\frac{\cot \theta}{R} J_{z^{\prime}}\right) .
$$

where $J_{x^{\prime}}, J_{y^{\prime}}, J_{z^{\prime}}$ are the components of the electron angular-momentum operator in the rotating coordinate system. Hence $\left[|m\rangle=\left|\varphi_{m}\left(\overrightarrow{\mathbf{r}}^{\prime}, R\right)\right\rangle\right]$

$$
\begin{aligned}
P_{m n}^{R} & \equiv P_{m n}^{z^{\prime}}=\left\langle m\left|-i \hbar \partial_{R}\right| n\right\rangle, \\
P_{m n}^{\theta} & \equiv P_{m n}^{x^{\prime}}=-\frac{1}{R}\left\langle m\left|J_{y^{\prime}}\right| n\right\rangle \\
P_{m n}^{\varphi} & \equiv P_{m n}^{y^{\prime}} \equiv \tilde{P}_{m n}^{\varphi}-\frac{\hbar \Lambda_{n} \cot \theta}{R} \delta_{m n} \\
& =\frac{1}{R}\left\langle m\left|J_{x^{\prime}}\right| n\right\rangle-\frac{\hbar \Lambda_{n} \cot \theta}{R} \delta_{m n}
\end{aligned}
$$

Here $\Lambda_{n} \hbar$ is the eigenvalue of $\varphi_{n}\left(\overrightarrow{\mathrm{r}}^{\prime}, R\right)$ with respect to $J_{z^{\prime}}$. Making the ansatz

$$
\chi_{n}(\overrightarrow{\mathrm{R}})=\frac{1}{R} \sum_{K, M} C_{n}^{K M} F_{n}^{K}(R) \mathcal{H}_{M \Lambda_{n}}^{K}(\theta, \varphi),
$$

where ${ }^{25}$

$$
\mathcal{H}_{M \Lambda_{n}}^{K}(\theta, \varphi)=[(2 K+1) / 4 \pi]^{1 / 2}(-i)^{\Lambda_{n}} e^{i M \varphi} d_{\Lambda_{n} M}^{K}(\theta)
$$

a re the eigenfunctions of the angular part of $\overrightarrow{\mathrm{P}}^{2}$, and projecting out $\mathcal{F}_{M \Lambda_{m}}^{* K}(\theta, \varphi)$, one obtains ${ }^{20,24}$ 


$$
\begin{aligned}
& \sum_{n}\left(\frac{1}{2 \mu}\left(-i \hbar \partial_{R} \underline{1}+\underline{\overrightarrow{\mathrm{P}}}^{R}+\underline{\overrightarrow{\mathrm{A}}}^{R}\right)_{m n}^{2}+\left[\epsilon_{n}(R)+V^{A B}(R)-E\right] \delta_{m n}+\frac{K(K+1) \hbar^{2}}{2 \mu R^{2}} \delta_{m n}+\Delta_{m n}\right. \\
& \left.\quad+\frac{1}{2 \mu}\left(\pi_{m n}^{2}+Q_{m n}^{K(+)}+Q_{m n}^{K(-)}\right)\right) C_{n}^{K M} F_{n}^{K}(R)=0 .
\end{aligned}
$$

Here $K(K+1) \hbar^{2} / 2 \mu R^{2}$ is the angular momentum barrier due to the rotation of the internuclear axis;

$$
\begin{aligned}
\pi_{m n}^{2} & =\left(\tilde{P}^{\varphi}\right)_{m n}^{2}+\left(P^{\theta}\right)_{m n}^{2}+\left(\tilde{P}^{\varphi} A^{\varphi}+A^{\varphi} \tilde{P}^{\varphi}+P^{\theta} A^{\theta}+A^{\theta} P^{\theta}\right)_{m n}-\frac{\Lambda_{n}^{2} \hbar^{2}}{R^{2}} \delta_{m n}-\left(A^{R}\right)_{m n}^{2}, \\
Q_{m n}^{K( \pm)} & =\delta_{\Lambda_{m}, \Lambda_{n} \pm 1} \frac{2 \hbar}{R}\left[\left(K \mp \Lambda_{n}\right)\left(K \pm \Lambda_{n}+1\right)\right]^{1 / 2}\left(P_{m n}^{\theta}+A_{m n}^{\theta}\right) \\
& =\delta_{\Lambda_{m}, \Lambda_{n} \pm 1} \frac{\hbar}{R}\left[\left(K \mp \Lambda_{n}\right)\left(K \pm \Lambda_{n}+1\right)\right]^{1 / 2}\left[P_{m n}^{\theta}+A_{m n}^{\theta} \mp i\left(\tilde{P}_{m n}^{\varphi}+A_{m n}^{\varphi}\right)\right] .
\end{aligned}
$$

$A_{m n}^{R} \equiv A_{m n}^{z^{\prime}}, A_{m n}^{\varphi} \equiv A_{m n}^{y^{\prime}}$, and $A_{m n}^{\theta} \equiv A_{m n}^{x^{\prime}}$ are the three components of the matrix vector $\overrightarrow{\mathrm{A}}_{m n}$ in the rotating coordinate system; they will be given explicitly in Eq. (3.29).

We will estimate the relative order of magnitude of the different terms in (2.53) below. It will turn out that the essential couplings between the molecular states are the modified radial coupling $\left(P_{m n}^{R}+A_{m n}^{R}\right)$ and the modified rotational coupling $Q_{m n}^{K( \pm)}$. In particular,

$$
P_{m n}^{R}+A_{m n}^{R}=-i \hbar\left\langle m\left|\partial_{R}\right| n\right\rangle+\frac{i}{\hbar} \mu\left(\epsilon_{m}-\epsilon_{n}\right)\left\langle m\left|X^{R}\right| n\right\rangle
$$

is the well-known radial coupling, corrected by a matrix element of the $R$ component of the translation vector $\overrightarrow{\mathrm{X}}[(2.25),(2.26)] . Q_{m n}^{K(t)}$ for $\Lambda_{n} \ll K$ approaches

$$
\frac{1}{2 \mu} Q_{m n}^{K(\star)} \cong-\delta_{\Lambda_{m^{\prime}} \Lambda_{n^{ \pm 1}}}\left(\frac{\hbar K}{\mu R^{2}}\left(J_{y^{\prime}}\right)_{m n}-\frac{\hbar K}{\mu R} A_{m n}^{x^{\prime}}\right)=-\omega_{y^{\prime}}\left[\left(J_{y^{\prime}}\right)_{m n}-R A_{m n}^{x^{\prime}}\right] \delta_{\Lambda_{m}, \Lambda_{n^{ \pm 1}}}
$$

i.e., the old rotational coupling $\vec{\omega} \cdot \vec{J}$, corrected by a matrix element of $\vec{X}$, too. Equation (2.53) may be rew ritten as

$\left(\frac{d^{2}}{d R^{2}}+k_{0}^{2}(R)\right) C_{n}^{K M} F_{n}^{K}(R)=-2 \sum_{n} D_{m n} C_{n}^{K M} \frac{d F_{n}^{K}}{d R}+\sum_{n}\left(B_{m n}+\frac{2 \mu}{\hbar^{2}}\left[\epsilon_{n}(R)-\epsilon_{0}(R)\right] \delta_{m n}\right) C_{n}^{K M} F_{n}^{K}(R)$

where

$$
\begin{aligned}
& D_{m n}=\frac{i}{\hbar}\left(P_{m n}^{R}+A_{m n}^{R}\right), \\
& B_{m n}=\frac{1}{\hbar^{2}}\left(\overrightarrow{\mathrm{P}}^{R}+\underline{\mathrm{A}}^{R}\right)_{m n}^{2}+\frac{2 \mu}{\hbar^{2}} \Delta_{m n}+\frac{1}{\hbar^{2}}\left(\pi_{m n}^{2}+Q_{m n}^{K( \pm)}\right),
\end{aligned}
$$

and

$$
k_{0}^{2}(R)=\frac{2 \mu}{\hbar^{2}}\left[E-\epsilon_{0}(R)-V^{A B}(R)\right]-\frac{K(K+1)}{R^{2}} .
$$

Here, $\epsilon_{0}(R)$ is a mean binding energy of the electrons independent of their state. In general one sets $\epsilon_{0}(R) \equiv 0$; then $k_{0}^{2}(R)$ corresponds to a Rutherford trajectory. Deviations of the nuclear trajectory from the Rutherford case due to the varying part of the total energy going into electronic binding energy ${ }^{26}$ may be considered here by introducing a convenient function $\epsilon_{0}(R)$. Effects of this kind, however, are small, ${ }^{26}$ and we will not consider them further.

The right-hand side of (2.58) describes the back reaction of electronic excitations on the nuclear (Rutherford) trajectory. The equations are diagonal in the total angular momentum $K$ and its projection $M$ with respect to $\overrightarrow{\mathrm{R}}$, because both quantities are conserved and not affected by excitations of the electron shell.

\section{THE SEMICLASSICAL APPROXIMATION OF THE NUCLEAR RELATIVE MOTION}

Any numerical solution of the quantum mechanical equations (2.58) would require taking into account many electronic states and, in general, even much more total angular momenta $K$. For heavy systems this will be completely impracticable. On the other hand, we expect that for heavy systems the nuclei will move along classical Rutherford trajectories without being much influenced by 
the electronic excitations. Thus the nuclear trajectory will to good degree of approximation be independent upon the state of the electron shell and only be determined by the total energy and total angular momentum. This approximation for the nuclear trajectory is obtained by applying the Jeffreys-Wentzel-Kramers-Brillouin (JWKB) approximation to Eqs. (2.58). This will be done in the following section using the methods of Refs. 27 and 28.

\section{A. The JWKB approximation}

Setting the right-hand side of $(2.58)$ equal to zero, the JWKB solutions are

$$
\begin{aligned}
& F_{m}^{K}(R)=A_{m}^{K}\left(\hbar k_{0}\right)^{-1 / 2} e^{ \pm(i / \hbar) S_{0}^{ \pm i \pi / 4}} \\
& S_{0}=\int_{R_{0}}^{R} \hbar k_{0}\left(R^{\prime}\right) d R^{\prime} .
\end{aligned}
$$

Here, $R_{0}$ is the classical turning point of the nuclei, where $k_{0}(R)$ becomes imaginary. $( \pm)$ denotes (out-, in-) going JWKB waves. The terms $\pm i \pi / 4$ in the exponent provide the correct adjustment at the point of closest approach. ${ }^{28.29}$

Now the full solutions of (2.58) may be expanded into this complete set of free JWKB waves. Then the dynamics of the expansion coefficients will be determined by the right-hand side of (2.58), i.e., the electronic excitations. We set

$$
\begin{aligned}
\tilde{F}_{n}^{K M}(R) \equiv & C_{n}^{K M} F_{n}^{K}(R) \\
= & \frac{1}{\left(\hbar k_{0}\right)^{1 / 2}}\left[a_{n}^{K M(+)}(R) e^{(i / \hbar)\left(s_{0}+\gamma_{n}\right)+i \pi / 4}\right. \\
& \left.-a_{n}^{K M(-)}(R) e^{-(i / \hbar)\left(S_{0}+\gamma_{n}\right)-i \pi / 4}\right],
\end{aligned}
$$

where

$$
\begin{aligned}
& \gamma_{n}=S_{n}-S_{0}=\int_{R_{0}}^{R} \hbar\left[k_{n}\left(R^{\prime}\right)-k_{0}\left(R^{\prime}\right)\right] d R^{\prime} \\
& k_{n}^{2}(R)=\frac{2 \mu}{\hbar^{2}}\left[E-\epsilon_{n}(R)-V^{A B}(R)\right]-\frac{K(K+1)}{R^{2}} .
\end{aligned}
$$

It is easy to show that

$$
\gamma_{n}=\mu \int_{R_{0}}^{R} \frac{\epsilon_{0}\left(R^{\prime}\right)-\epsilon_{n}\left(R^{\prime}\right)}{\hbar k_{0}\left(R^{\prime}\right)} d R^{\prime}+O\left(\frac{\epsilon_{n}^{2}}{E^{2}}\right) \text {. }
$$

One of the two parameters $a_{n}^{K M( \pm)}(R)$ in (3.2) can be eliminated by demanding ${ }^{27}$

$$
\begin{aligned}
& \frac{d a_{m}^{K M(+)}}{d R} e^{(i / \hbar)\left(S_{0}+\gamma_{m}\right)+i \pi / 4} \\
& =\frac{d a_{m}^{K M(-)}}{d R} e^{-(i / \hbar)\left(s_{0}+\gamma_{m}\right)-i \pi / 4},
\end{aligned}
$$

which eliminates all second derivatives of $a_{m}^{K M(t)}$ in the equations of motion.

We now insert the ansatz (3.2) into (2.58), expand in terms of $\hbar$ (semiclassical approximation), and neglect all terms containing strongly oscillating phase factors. ${ }^{27,29}$ Then the equations of motion separate into two sets of equations for $a_{n}^{K M(+)}(R)$ and $a_{n}^{K M(-)}(R)$ (Ref. 27):

$$
\begin{aligned}
2 i \hbar p_{m} a_{m}^{K M(+)^{\prime}} & =\sum_{n}\left(2 p_{n} \tilde{D}_{m n}+\hbar^{2} B_{m n}\right) a_{n}^{K M(+)} \exp \left(i / \hbar \int_{R_{0}}^{R}\left(\epsilon_{m}-\epsilon_{n}\right) \frac{\mu}{p_{0}} d R^{\prime}\right), \\
2 i \hbar p_{m} a_{m}^{K M(-)^{\prime}} & =\sum_{n}\left(2 p_{n} \tilde{D}_{m n}-\hbar^{2} B_{m n}\right) a_{n}^{K M(-)} \exp \left(-i / \hbar \int_{R_{0}}^{R}\left(\epsilon_{m}-\epsilon_{n}\right) \frac{\mu}{p_{0}} d R^{\prime}\right),
\end{aligned}
$$

where the prime denotes derivative with respect to $R ; p_{m}=\hbar k_{m}$ and $\tilde{D}_{m n}=-i \hbar D_{m n}$.

\section{B. The time-dependent coupled-channel equations}

$a_{n}^{K M(+)}$ is the coefficient of the outgoing JWKB wave. Define in the outgoing channel

$$
t=\int_{R_{0}}^{R} \frac{\mu}{p_{0}\left(R^{\prime}\right)} d R^{\prime}, \quad t \geqslant 0 .
$$

This specifies a classical trajectory $R(t)$. Now (3.7) may be rewritten into a time-dependent set of equations:

$$
\dot{a}_{m}^{K M(+)}(t)=\sum_{n}\left[-\frac{i}{\hbar} \frac{p_{0}}{p_{m}}\left(\frac{p_{n}}{\mu} \tilde{D}_{m n}+\tilde{B}_{m n}\right)\right] a_{n}^{K M(+)}(t) \exp \left(-\frac{i}{\hbar} \int_{0}^{t}\left(\epsilon_{n}-\epsilon_{m}\right) d t^{\prime}\right),
$$

where

$$
\tilde{B}_{m n}=\left(\hbar^{2} / 2 \mu\right) B_{m n} \text {. }
$$

For the ingoing channel we define

$$
t=-\int_{R_{0}}^{R} \frac{\mu}{p_{0}\left(R^{\prime}\right)} d R^{\prime}, \quad t \leqslant 0
$$


so that (3.8) becomes

$$
\dot{a}_{m}^{K M(\rightarrow}(t)=\sum_{n}\left[\frac{i}{\hbar} \frac{p_{0}}{p_{m}}\left(\frac{p_{n}}{\mu} \tilde{D}_{m n}-\tilde{B}_{m n}\right)\right] a_{n}^{K M(-)}(t) \exp \left(-\frac{i}{\hbar} \int_{0}^{t}\left(\epsilon_{n}-\epsilon_{m}\right) d t^{\prime}\right) .
$$

In order to get a regular function $F_{m}^{K}(R)$ at $R=R_{0}(t=0)$ we demand 27,28

$$
a_{m}^{K M(+)}(t=0)=a_{m}^{K M(\rightarrow)}(t=0) .
$$

Hence we may define

$$
a_{m}^{K M}(t)= \begin{cases}a_{m}^{K M(+)}(t), & t \geqslant 0 \\ a_{m}^{K M(-)}(t), & t \leqslant 0\end{cases}
$$

in order to get

$$
\dot{a}_{m}^{K N}(t)=-\frac{i}{\hbar} \frac{p_{0}}{p_{m}} \sum_{n}\left(\operatorname{sgn}(t) \frac{p_{n}}{\mu} \tilde{D}_{m n}+\tilde{B}_{m n}\right) a_{n}^{K M}(t) \exp \left(-\frac{i}{\hbar} \int_{0}^{t}\left(\epsilon_{n}-\epsilon_{m}\right) d t^{\prime}\right) .
$$

Below we will show that these coupled-channel equations are very similar to the ones obtained from PSS theory. However, they contain corrections which just cancel the unwanted spurious, long-range couplings of the PSS theory. Thus we have derived from a consistent quantum-mechanical treatment of the scattering problem the correct coupled-channel equations for the electronic occupation amplitudes by applying the semiclassical approximation.

Before proving this statement in detail, we want to discuss, shortly, the structure of Eqs. (3.16). $a_{m}^{K / M}\left(t_{0}\right)$ is interpreted as the amplitude for the situation that in a scattering process with total energy $E$ and total angular momentum $K$ (i.e., with a given impact parameter $b$ ) and a given projection $M$ the electrons are in state $\varphi_{m}\left(\overrightarrow{\mathrm{r}}, R\left(t_{0}\right)\right)$ at time $t=t_{0}$. These amplitudes are determined by all other amplitudes at all times $\leqslant t_{0}$. In particular, excitations during the ingoing channel will interfere with excitations in the outgoing channel. This complete coherence contained in (3.16) is due to the JWKB approximation. We have expanded with respect to JWKB waves with definite energy $E$, which therefore are spread over all values of $R$ (i.e., over the whole trajectory). In order to destroy the coherence, we should have to expand with respect to localized wave packets for the nuclear motion. Our derivation, however, yields complete coherence.

Finally, we want to say a few words about the validity of the JWKB approximation. Using the well-known criterion ${ }^{27,28}$

$$
p^{-3 / 2} \frac{d^{2}}{d R^{2}} p^{-1 / 2} \ll 1
$$

one can show ${ }^{24}$ that this is true for

$$
\frac{R-R_{0}}{R_{0}} \gtrsim \begin{cases}\chi^{-2 / 3}, & \hbar K \text { small } \\ K^{-2 / 3}, & \hbar K \text { large }\end{cases}
$$

where

$$
\chi=\frac{Z_{A} Z_{B} e^{2}}{\hbar(2 E / \mu)^{1 / 2}} \cong \frac{Z_{A} Z_{B} e^{2}}{\hbar v}
$$

is the Sommerfeld parameter, which for $\mathrm{Pb}-\mathrm{Pb}$ collisions at $v=0.1 c\left(E_{1 \mathrm{ab}}=4.7 \mathrm{MeV} / \mathrm{amu}\right)$ is $\chi$ $\cong 490$. In such a collision the JWKB approximation breaks down at $R-R_{0} \gtrsim 0.015 R_{0}$, which is of the order of $0.2 \mathrm{fm}$. The errors due to this breakdown of the classical trajectory picture near the classical turning point in the calculation of the amplitudes $a_{n}^{K M}(t)$ may be safely neglected.

\section{Comparison with PSS theory}

We now will compare Eq. (3.16) with the corresponding result (1.4) of PSS theory. Note that

$$
\begin{aligned}
& \frac{p_{0}}{p_{m}}=1+O\left(\frac{\epsilon_{m}-\epsilon_{0}}{E}\right)=1+O\left(\frac{m}{\mu}\right) \\
& \frac{p_{n} p_{0}}{\mu p_{m}}=\frac{p_{0}}{\mu}\left[1+O\left(\frac{m}{\mu}\right)\right]=\operatorname{sgn}(t) \dot{R}(t)\left[1+O\left(\frac{m}{\mu}\right)\right] .
\end{aligned}
$$

The corrections $\sim m / \mu$ are due to the assumption of a unique classical trajectory (independent of the electronic state); in reality, systems in different electronic states move on slightly different trajectories. This effect in (3.16) shows up as additional couplings. Neglecting these corrections and dropping the indices $K$ and $M$, we obtain from (3.16) and (2.59)

$$
\dot{a}_{m}(t)=-\sum_{n}\left[\dot{R}\left(\left\langle m\left|\partial_{R}\right| n\right\rangle+\frac{i}{\hbar} A_{m n}^{R}\right)+\frac{i}{\hbar} \tilde{B}_{m n}\right] a_{n}(t) e^{-i \times n m(t)},
$$


where

$$
\chi_{n m}(t) \equiv \frac{1}{\hbar} \int_{0}^{t}\left(\epsilon_{n}-\epsilon_{m}\right) d t^{\prime}
$$

Hence the radial coupling $\dot{R} \partial_{R}$ of PSS theory is contained in our theory as well, corrected, however, by the term $\dot{R}(i / \hbar) A_{m n}^{R}$. Since, according to our considerations in Sec. IID, the sum $\left(\partial_{R}\right)_{m n}+(i / \hbar) A_{m n}^{R}$ asymptotically vanishes, our theory does not contain any spurious long-range radial couplings. Now let us study the four couplings generated by

$$
\tilde{B}_{m n}=\frac{1}{2 \mu}\left(\underline{\mathbf{P}}^{R}+\underline{\overrightarrow{\mathrm{A}}}^{R}\right)_{m n}^{2}+\Delta_{m n}+\frac{1}{2 \mu}\left[\pi_{m n}^{2}+Q_{m n}^{K(t)}\right]
$$

and compare them in their order of magnitude with the radial coupling

$$
\begin{aligned}
& \frac{i}{\hbar} \dot{R}\left\langle m\left|P^{R}\right| n\right\rangle \sim \dot{R}(m \dot{R})=2 \frac{m}{\mu} \frac{\mu}{2} \dot{R}^{2} . \\
& \text { (i) } \frac{\left(\overrightarrow{\mathrm{P}}^{R}\right)_{m n}^{2}}{2 \mu} \sim \frac{1}{2 \mu}(m \dot{R})^{2}=\left(\frac{m}{\mu}\right)^{2}\left(\frac{\mu}{2} \dot{R}^{2}\right),
\end{aligned}
$$

and hence $(1 / 2 \mu)\left(\underline{\overrightarrow{\mathrm{P}}}+\underline{\overrightarrow{\mathrm{A}}}^{R}\right)_{m n}^{2}$ are by an order $m / \mu$ smaller than (3.25).

(ii) $\frac{\pi_{m n}^{2}}{2 \mu}=\left(2 \mu R^{2}\right)^{-1}\left\langle m\left|J_{x^{\prime}}^{2}+J_{y^{\prime}}^{2}\right| n\right\rangle+(2 \mu R)^{-1}\left\langle m\left|J_{x^{\prime}} A^{y^{\prime}}+A^{y^{\prime}} J_{x^{\prime}}-J_{y^{\prime}} A^{x^{\prime}}-A^{x^{\prime}} J_{y^{\prime}}\right| n\right\rangle-(2 \mu)^{-1}\left(A^{R}\right)_{m n}^{2}$.

The last term is of the order of (3.26). The other terms are of the order $J_{e}^{2} / 2 \mu R^{2} \ll \overrightarrow{\mathrm{I}} \cdot \vec{J}_{e} / 2 \mu R^{2}$, which is much smaller than the coriolis coupling $Q_{m n}^{K( \pm)}$ given below in (3.27), since the electron angular momentum $J_{e}$ is much smaller than the total angular momentum $I . \quad\left(J_{e} \lesssim 10 \hbar\right.$, whereas in $\mathrm{Pb}-\mathrm{Pb}$ collisions at the Coulomb barrier $I \sim 100-1000 \hbar$.)

(iii) From (2.57) we know that $Q_{m n}^{K( \pm)}$ is the modified rotational coupling which is of the order

$$
\begin{aligned}
\frac{\overrightarrow{\mathrm{I}} \cdot \overrightarrow{\mathrm{J}}_{e}}{2 \mu R^{2}} & =\vec{\omega} \cdot \overrightarrow{\mathrm{J}}_{e} \cdot \\
\text { (iv) } \Delta_{m n} & =\langle m|V(\overrightarrow{\mathrm{r}}, \overrightarrow{\mathrm{R}}-\overrightarrow{\mathrm{X}})-V(\overrightarrow{\mathrm{r}}, \overrightarrow{\mathrm{R}})| n\rangle \cong-\frac{m}{\mu}\left\langle m\left|\sum_{l=1}^{\mathbf{Z}^{\prime}} \overrightarrow{\mathrm{s}}_{l} \cdot \vec{\nabla}_{R} V(\overrightarrow{\mathrm{r}}, \overrightarrow{\mathrm{R}})\right| n\right\rangle .
\end{aligned}
$$

Splitting $V(\overrightarrow{\mathrm{r}}, \overrightarrow{\mathrm{R}})$ into $V^{A B}(R)+V_{\mathrm{mol}}^{\mathrm{el}}(\overrightarrow{\mathrm{r}}, \overrightarrow{\mathrm{R}})$ and using $\vec{\nabla}_{R} V_{\mathrm{mol}}^{\mathrm{el}}=\vec{\nabla}_{R} H_{\mathrm{mol}}^{\mathrm{el}}$ and

$$
\overrightarrow{\mathrm{A}}_{m k}=\frac{i}{\hbar} \mu\left\langle m\left|\left[H_{\mathrm{mol}}^{\mathrm{el}}, \overrightarrow{\mathrm{X}}\right]\right| k\right\rangle=\frac{i}{\hbar} m\left(\epsilon_{m}-\epsilon_{k}\right)\left\langle m\left|\sum_{l}^{Z^{\prime}} \overrightarrow{\mathrm{s}}_{l}\right| k\right\rangle \text {, }
$$

$\Delta_{m n}$ can be rewritten as

$$
\Delta_{m n} \cong \frac{i \hbar}{\mu} \sum_{k} \frac{\overrightarrow{\mathrm{A}}_{m k}}{\epsilon_{m}-\epsilon_{k}} \cdot\left[\left\langle\boldsymbol{k}\left|\vec{\nabla}_{R} H_{\mathrm{mol}}^{\mathrm{el}}\right| n\right\rangle+\left\langle k\left|\vec{\nabla}_{R} V^{A B}\right| n\right\rangle\right]
$$

By the Hellman-Feynman theorem,

$$
\left\langle k\left|\vec{\nabla}_{R} H_{\mathrm{mol}}^{\mathrm{el}}\right| n\right\rangle=\delta_{k n} \vec{\nabla}_{R} \epsilon_{n}+\frac{i}{\hbar}\left(\epsilon_{n}-\epsilon_{k}\right) \overrightarrow{\mathrm{P}}_{k n}
$$

we find

$$
\Delta_{m n} \cong \frac{m}{\mu}\left\langle m\left|\sum_{l} \overrightarrow{\mathrm{s}}_{l}\right| n\right\rangle \cdot\left(\vec{\nabla}_{R} \epsilon_{n}+\vec{\nabla}_{R} V^{A B}\right)-\frac{1}{\mu} \sum_{k} \frac{\epsilon_{n}-\epsilon_{k}}{\epsilon_{m}-\epsilon_{k}} \overrightarrow{\mathrm{A}}_{m k} \cdot \overrightarrow{\mathrm{P}}_{k n}
$$

The second term is of order (3.26), which is small compared to the radial coupling. According to the Hellman-Feynman theorem, the very first term is of the same order of magnitude. Since the electron binding energies roughly vary like $V^{A B}$, the term $\sim \vec{\nabla}_{R} V^{A B}$ is also of this order. Hence $\Delta_{m n}$ is small compared to the radial coupling (3.25).

Taking into account only those terms of $\tilde{B}_{m n}$ which are comparable to the radial coupling, and neglecting terms smaller by a factor $m / \mu$ or $J_{e} / I$, the coupled-channel equations read

$$
\dot{a}_{m}(t)=-\sum_{n}\left[\dot{R}\left(\left\langle m\left|\partial_{R}\right| n\right\rangle+\frac{i}{\hbar} A_{m n}^{R}\right)-\frac{i}{\hbar} \delta_{\Lambda_{m}, \Lambda_{n^{ \pm 1}}} \frac{\hbar K}{\mu R^{2}}\left[\left(J_{y^{\prime}}\right)_{m n}-R A_{m n}^{x^{\prime}}\right]\right] a_{n}(t) e^{-i \times{ }_{n m}(t)} .
$$


To this order the only corrections to PSS theory are matrix elements of the translation vector $\overrightarrow{\mathrm{X}}$ :

$$
\overrightarrow{\mathrm{A}}_{m n}=\frac{i}{\hbar} \mu\left(\epsilon_{m}-\epsilon_{n}\right)\langle m|\overrightarrow{\mathrm{X}}| n\rangle \text {. }
$$

They are not hard to calculate if the molecular wave functions are known. On the other hand, we can continue using the tabulated molecular wave functions $\varphi_{m}$, the radial and rotational coupling matrix elements of PSS theory $.5,21,30$ This is the essential advantage of this approach, as was first pointed out by Thorson and Delos.20

\section{The cross section in the semiclassical approximation}

From (3.2) one can calculate the amplitude for the electrons being scattered from state $m_{0}$ to state $n$ in a collision with total angular momentum $(K, M)$. If for $t \rightarrow-\infty$ the electrons were in state $\varphi_{m_{0}}$, we would have

$$
a_{m}^{K M}(t=-\infty)=a_{m}^{K M}(-)(R=\infty)=\delta_{m m_{0}}
$$

Hence

$$
\left.\tilde{F}_{n}^{K M}(R \rightarrow \infty)-\frac{1}{\sqrt{\hbar k_{0}}} a_{n}^{K M /(+)}(R=\infty) e^{(i / \hbar)\left(p_{n} R-\hbar K \pi / 2\right)} e^{i \sigma_{n}}-\delta_{n m_{0}} e^{-(i / \hbar)\left(p_{m_{0}} R-\hbar K \pi / 2\right)} e^{-i \sigma_{m_{0}}}\right],
$$

where we have introduced the phase shift

$$
\sigma_{n}=\lim _{R \rightarrow \infty}\left(\frac{1}{\hbar} \int_{R_{0}}^{R} \hbar k_{n}\left(R^{\prime}\right) d R^{\prime}-k_{n} R+\frac{K \pi}{2}\right) .
$$

Following Ref. 27, we may read off the scattering matrix element in the rotating basis:

$$
S_{m_{0} \rightarrow n}^{K M}=a_{n}^{K M}(t=\infty)\left(\frac{p_{n}}{p_{m_{0}}}\right)^{1 / 2} e^{i\left(\sigma_{n}+\sigma_{m_{0}}\right)} .
$$

The total cross section in the laboratory system (i.e., nonrotating system) is obtained from (3.39) by multiplying with the angular eigenfunctions $\mathcal{H}_{M \Lambda_{m_{0}}}^{* K}, \mathfrak{H}_{M \Lambda_{n}}^{K}$, averaging over $M$, summing over all $K$, and taking the square. We will not present the explicit calculation which is done by rewriting the sum over $K$ into an integral and applying the saddle-point method. ${ }^{28,31}$ The saddle point $K_{0}$ lies in the neighborhood of the value $K$ corresponding to the scattering angle $\theta$ via the classical Rutherford trajectory. The deviations are the smaller, the larger $K_{0}$ is. Since, for large $K_{0}$, the contributions from angular momenta neighboring $K_{0}$ very soon interfere destructively, the saddle point is the sharper the larger $K_{0}$. Thus for large $K_{0}$ the integrand is only different from zero in a region where $a_{n}^{K M}(\infty)$ only slightly varies. Therefore,

$$
\left[1 /\left(2 K_{0}+1\right)\right] \sum_{M} a_{n}^{K_{0} M}(\infty)
$$

can be written in front of the $K$ integral. The remaining integral does not contain any amplitudes $a_{n}^{K M}(\infty)$, but only contributions from the nuclear trajectory, and hence just yields the Rutherford cross section due to the JWKB approximation [respectively, if $\epsilon_{0}(R) \neq 0$, a modified Rutherford cross section $\left.{ }^{26}\right]$. As a result we have

$$
\frac{d \sigma}{d \Omega}{ }_{m_{0} \rightarrow n}=\left|a_{n}^{K}(\infty)\right|_{K=a \cot \theta / 2}^{2}\left(\frac{d \sigma}{d \Omega}\right)_{\text {Ruth }},
$$

where $a=(P / 2 E) Z_{A} Z_{B} e^{2}$ and $a_{n}^{K}$ is the $M$-averaged amplitude. We wish to stress that this derivation not only makes use of the JWKB approximation, but in addition assumes that the total angular momentum of the nuclear trajectory is very large. This is not true for proton-hydrogen collisions at energies $\sim 500 \mathrm{eV}$ although the Sommerfeld parameter is large. In this case (3.40) leads to wrong results. ${ }^{31}$

\section{HOW TO DESCRIBE CONTINUUM ELECTRONS}

From the derivations of Secs. II and III it follows that the matrix elements $\overrightarrow{\mathrm{A}}_{m n}$ asymptotically cancel the spurious dynamical couplings only if one or both of the two states $\varphi_{n}, \varphi_{m}$ for $R \rightarrow \infty$ separate into a product of atomic states according to Eq. (2.9). [Because of the Hermiticity of $H^{\prime}(\vec{r}, \vec{R})$ it suffices, if (2.9) is true for one of the two states.] All bound states (i.e., all states not containing an electron with $|\epsilon| \geqslant m_{0} c^{2}$ ) fulfill this condition because they asymptotically become localized around the nuclei, whereas the essential interaction between the ions vanishes. On the other hand, one easily realizes that continuum electrons in an energy eigenstate do not possess this property (2.9); the corresponding wavefunctions (for example, two-center Coulomb wavefunctions with an energy $\hbar \omega)$ are spread over all space with an amplitude decaying only as $1 / r$ as $r \rightarrow \infty$. Therefore, these wavefunctions, even for a single electron, always feel the potential of both nuclei, i.e., the asymptotic Coulomb phase shift depends upon 
both nuclear fields and, in particular, upon the distance $R$ between the two nuclei. ${ }^{32}$ The twocenter Coulomb waves therefore will never be expressible in terms of $\vec{r}_{A}^{*}$ or $\vec{r}_{B}^{*}$ alone, but only as a function of both, and hence of $\vec{R}$ or $\vec{R}_{A B}$, respectively. This is the reason for $\overrightarrow{\mathrm{P}}_{m n}$ couplings between the Coulomb waves which cannot be suppressed by our translation matrix elements $\overrightarrow{\mathrm{A}}_{m n}$.

Hence for continuum electrons our idea with the electron translation operator does not work. On the other hand, physical intuition tells us that the continuum electrons (moving already with nearly the velocity of light for kinetic energies $z 10 \mathrm{keV}$ ) will quickly leave the region of influence by the two-center potential. Therefore, no asymptotic excitations should occur. Obviously, the twocenter basis is not appropriate to describe this behavior. The elementary reason is that electrons leaving the interaction region must be described by spatially localized wave packets instead of Coulomb waves which are spread over all space. Using a basis of wave packets, we may hope to escape the asymptotic couplings.

Unfortunately, the construction of such a basis is not at all elementary. First of all, there exist no continuum solutions of the two-center Dirac equation in the literature from which we could construct our wave packets. Furthermore, it turns out to be rather difficult to construct a complete orthogonal basis with the desired localization properties. Therefore, we will not use the two-center continuum, but consider a simpler possibility. We shall use a basis which by construction asymptotically approaches an atomic continuum basis and thus avoids the problem of spurious long-range couplings. We only have to show that in this basis all other coupling matrix elements are finite and vanish for $R \rightarrow \infty$. Questions of completeness will be considered too.

Before going into the details, let us express some words of motivation. Why are we interested in such a thorough description of the continuum? As calculations by Soff, Reinhardt, and other $\mathrm{s}^{15,33,34}$ showed, a much simpler treatment is sufficient to describe ionization cross sections $^{35}$ and energy spectra of $\delta$ electrons. ${ }^{36,37}$ However, there is one measurable entity which cannot be obtained with the monopole approximation for the two-center potential used by these authors, i.e., the angular distribution of $\delta$ electrons. ${ }^{36,37}$ In order to be able to compare this entity in theory and experiment, an exact theoretical treatment of the continuum in the field of two nuclei becomes necessary. In Sec. IV $\mathrm{E}$ we will show how within the framework to be presented below angular distributions of $\delta$ electrons may be calculated.

\section{A. The basis for the continuum electrons}

In the following formulation, bound and continuum electrons will be described by different basis sets. Whereas for the bound electrons we shall use the molecular basis for which we developed the translation-operator formalism, the continuum electrons will be treated in a new quasiatomic basis which for $R \rightarrow \infty$ approaches an atomic basis around nucleus $A(B)$ and for which, therefore, the application of translation operators is not necessary. Of course, the new basis will not be orthogonal on the molecular bound states and, in addition, there will occur further coupling matrix elements from the two-center potential. All these points will be studied.

In order to be able to split the electron configuration into a bound part and a continuum part, we must neglect the recoil term $\left(1 / 2 M_{N}\right)\left(\sum \vec{p}_{i}\right)^{2}$ as well as the nondiagonal part of the electron interaction. Then the electron configuration may be written as a Slater determinant:

$$
\varphi_{m}\left(\overrightarrow{\mathrm{r}}_{1}, \cdots, \overrightarrow{\mathrm{r}}_{z^{\prime}} ; \overrightarrow{\mathrm{R}}\right)=\mathcal{Q}\left[\varphi_{m_{1}}\left(\overrightarrow{\mathrm{r}}_{1} ; \overrightarrow{\mathrm{R}}\right) \cdots \varphi_{m_{K}}\left(\overrightarrow{\mathrm{r}}_{K} ; \overrightarrow{\mathrm{R}}\right) \tilde{\varphi}_{\omega_{K+1}}\left(\overrightarrow{\mathrm{r}}_{K+1} ; \overrightarrow{\mathrm{R}}\right) \cdots \tilde{\varphi}_{\omega_{z^{\prime}}}\left(\overrightarrow{\mathrm{r}}_{z^{\prime}} ; \overrightarrow{\mathrm{R}}\right)\right]
$$

Here, $Q$ is the antisymmetrization operator, $K$ is the number of bound electrons, $\varphi_{m_{i}}\left(\overrightarrow{\mathbf{r}}_{i} ; \overrightarrow{\mathrm{R}}\right)$ are bound molecular one-electron states with energies $\left|\epsilon_{i}(R)\right|<m_{0} c^{2}$, and the $\tilde{\varphi}_{\omega_{i}}\left(\overrightarrow{\mathrm{r}}_{i} ; \overrightarrow{\mathrm{R}}\right)$ are the one-electron continuum states with energies $\left|\hbar \omega_{i}\right| \geqslant m_{0} c^{2}$ to be specified now.

For the construction of the continuum basis we applied the following criteria. In order to guarantee asymptotically the property $(2.9)$ it would be simplest to choose wave functions $\tilde{\varphi}_{\omega}$ which in the limit $R \rightarrow \infty$ become atomic (one-center) Coulomb waves around nucleus $A$ or $B$. In the molecular limit, however, the electrons should feel the total nuclear charge $Z_{A}+Z_{B}$. Furthermore, in order to avoid undesired couplings in the molecular limit by the potential of nucleus $B$, if $\tilde{\varphi}_{\omega}$ is a continuum state around nucleus $A$, the quasiatomic potential generating $\tilde{\varphi}_{\omega}$ should be located in the nuclear center of mass $\overrightarrow{\mathrm{r}}=0$ for $R \rightarrow 0$. In that case, for $R \rightarrow 0$ the $\tilde{\varphi}_{\omega}$ are quasiatomic monopole waves for $Z_{A}+Z_{B}$, and there only remain coupling matrix elements due to the higher multipole contributions of the two-center potential.

For the following we must specify whether the 
$\tilde{\varphi}_{\omega}$ asymptotically belong to nucleus $A$ or $B$. Since all electrons (positrons) with energy not too little above $|\hbar \omega|=m_{0} c^{2}$ quickly leave the region of interaction with the two nuclei, this choice should not influence the results too strongly. Thus we arbitrarily choose nucleus $A$ :

$$
\tilde{H}^{A}(\vec{\rho}, R)=\vec{\alpha} \cdot \overrightarrow{\mathrm{p}}_{\rho} c+\beta m_{0} c^{2}+\tilde{V}(\rho),
$$

where

$$
\vec{\rho}=\overrightarrow{\mathrm{r}}-g(R) \overrightarrow{\mathrm{R}} \rightarrow\left\{\begin{array}{l}
\overrightarrow{\mathbf{r}}, \text { for } R \rightarrow 0 \\
\overrightarrow{\mathbf{r}}_{A}=\overrightarrow{\mathbf{r}}+\frac{M_{B}}{M_{N}} \overrightarrow{\mathrm{R}}, \text { for } R \rightarrow \infty
\end{array}\right.
$$

and

$$
\tilde{V}(\rho)= \begin{cases}-\frac{\left(Z_{A}+\tilde{g}(R) Z_{B}\right) e^{2}}{\rho} & \rho \geqslant \frac{1}{2} \tilde{g}(R) R \\ -\frac{2\left(Z_{A}+\tilde{g}(R) Z_{B}\right) e^{2}}{\tilde{g}(R) R}, & \rho<\frac{1}{2} \tilde{g}(R) R\end{cases}
$$

$\overrightarrow{\mathrm{p}}_{\rho}=-i \hbar \vec{\nabla}_{\rho}$ is the canonically conjugate momentum for $\vec{\rho}$. The potential $\tilde{V}(\rho)$ for $R \rightarrow 0$ contains the monopole part of the two-center potential (as used by Soff and Reinhardt ${ }^{5,14,15}$ ) up to small terms stemming from the mass asymmetry and remaining finite. [Equation (4.4) for $R \rightarrow 0$ yields the monopole part of the two-center potential for point nuclei. In order to get for small $R$ the monopole part for extended nuclei, ${ }^{5} \tilde{g}(R)$ may be defined appropriately.] In Fig. 2 the definition of

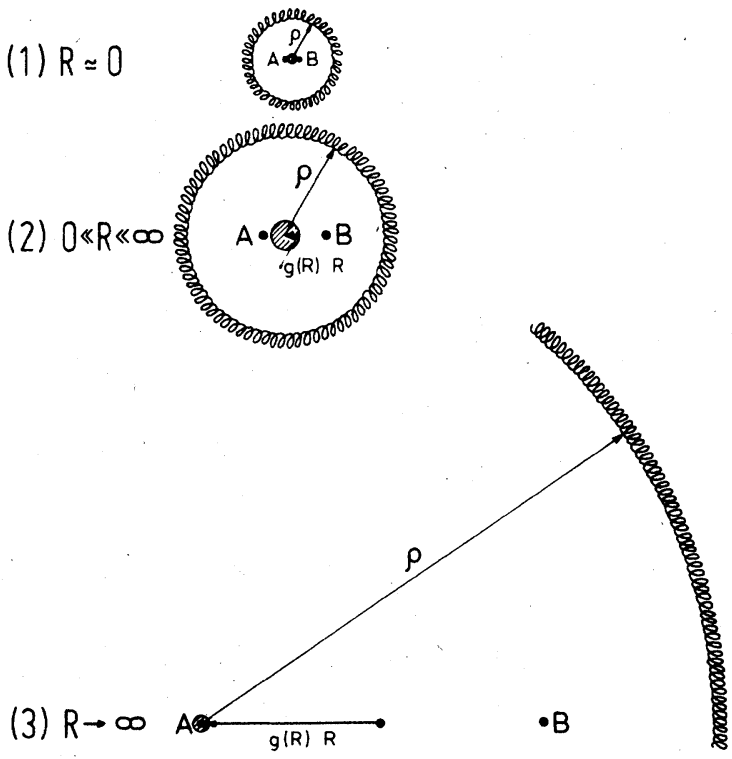

FIG. 2. The quasiatomic potential $\tilde{V}(\rho)$ generating the quasiatomic continuum basis $\tilde{\varphi}_{\omega}^{A}$, schematically depicted for different nuclear distances $R$.
$\tilde{V}(\rho)$ is demonstrated pictorially.

The functions $g(R), \tilde{g}(R)$ are to obey the boundary conditions:

$$
\begin{aligned}
& g(R) \underset{R \rightarrow 0}{\longrightarrow} 0 ; g(R) \underset{R \rightarrow \infty}{\longrightarrow}-\frac{M_{B}}{M_{N}} \\
& \tilde{g}(R) \underset{R \rightarrow 0}{\longrightarrow} 1 ; \tilde{g}(R) \underset{R \rightarrow \infty}{\longrightarrow} 0 .
\end{aligned}
$$

As a continuum basis we choose the eigenstates of $\tilde{H}^{A}(\vec{\rho}, R)$ :

$$
\tilde{H}^{A}(\vec{\rho}, R) \tilde{\varphi}_{\omega}^{A}(\vec{\rho}, R)=\hbar \omega \tilde{\varphi}_{\omega}^{A}(\vec{\rho}, R) ; \quad|\hbar \omega| \geqslant m_{0} c^{2} .
$$

These are usual relativistic one-center Coulomb wavefunctions. ${ }^{38}$ Their asymptotic behavior is given by $\left[\delta_{k}(\rho, \omega)\right.$ is the logarithmically increasing Coulomb phase]:

$$
\tilde{\varphi}_{\omega}^{A}(\vec{\rho}, R) \underset{\rho \rightarrow \infty}{\longrightarrow}\left[\begin{array}{c}
\left(\frac{\hbar \omega+m_{0} c^{2}}{\pi p c}\right)^{1 / 2} \frac{1}{\rho} \cos \left[p \rho+\delta_{\kappa}(\rho, \omega)\right] \chi_{\kappa}^{\mu} \\
\left(\frac{\hbar \omega-m_{0} c^{2}}{\pi p c}\right)^{1 / 2} \frac{1}{\rho} \sin \left[p \rho+\delta_{\kappa}(\rho, \omega)\right] \chi_{-\kappa}^{\mu}
\end{array}\right] .
$$

Hence $\tilde{\varphi}_{\omega}^{A}$ oscillates with a wavelength $\lambda \leqslant 2 \pi x_{e}$ $=2426 \mathrm{fm}$ and with an amplitude decreasing like $1 / \rho$. This decrease of the amplitude, however, is too weak to make the interaction of $\tilde{\varphi}_{\omega}^{A}$ with the potential of nucleus $B$ vanish for $R \rightarrow \infty$. In order to achieve the latter, we must construct wave packets from the $\tilde{\varphi}_{\omega}^{A}$ which decay faster than $1 / \rho$. In general such a wave packet reads as follows:

$$
\Psi_{E}(\vec{\rho}, \overrightarrow{\mathrm{R}}, t)=\int c_{E}(\omega) \tilde{\varphi}_{\omega}^{A}(\vec{\rho}, R) e^{-i \omega t} d \omega
$$

This is not an eigenstate of $\tilde{H}^{A}$; its mean energy is given by

$$
E=\left\langle\Psi_{E}\left|\tilde{H}^{A}\right| \Psi_{E}\right\rangle=\int \hbar \omega\left|c_{E}(\omega)\right|{ }^{2} d \omega
$$

The $\tilde{\varphi}_{\omega}^{A}$ are normalized to $\delta\left(\omega-\omega^{\prime}\right)$; thus the normalization relation for the $\Psi_{E}$ reads as

$$
\left\langle\Psi_{E} \mid \Psi_{E^{\prime}}\right\rangle=\int c_{E}^{*}(\omega) c_{E^{\prime}}(\omega) d \omega=\delta_{E E^{\prime}} .
$$

This condition is easily satisfied by the so-called Weyl packets:

$\Psi_{E}(\vec{\rho}, \overrightarrow{\mathrm{R}}, t)=\frac{1}{(\Delta \omega)^{1 / 2}} \int_{E_{-}=E-n \Delta \omega / 2}^{E_{+}=E+n \Delta \omega / 2} \tilde{\varphi}_{\omega}^{A}(\vec{\rho}, R) e^{-i \omega t} d \omega$,

if for different states the energy intervals are chosen disjoint, $\left|E^{\prime}-E\right| \geqslant \hbar \Delta \omega$. Then, however, the Weyl packets are no longer complete, since 


$$
\sum_{E} c_{E}^{*}(\omega) c_{E}\left(\omega^{\prime}\right) \neq \delta\left(\omega-\omega^{\prime}\right) .
$$

We will discuss below the practical importance of this incompleteness.

The amplitude of the Weyl packets $\tilde{\varphi}_{E}$ decay like $1 / \rho^{2}$ (see Sec. IV C). This turns out to be sufficient to make the couplings with the other nucleus vanish for $R \rightarrow \infty$.

In Fig. 3 we show a wave packet which we obtained numerically by superimposing one-center Coulomb waves according to (4.12). The mean energy of the packet is $2.15 m_{0} c^{2}$; its width $\hbar \Delta \omega$ $=0.30 m_{0} c^{2}$. The energy integral was done by 12-point Gauss integration. The relative numerical error is everywhere less than $10^{-4}$. One realizes the $1 / \rho$ decrease of the amplitude of $\rho \psi_{E}$ in the figure; i.e., the wave packet falls off like $1 / \rho^{2}$ as it should do.

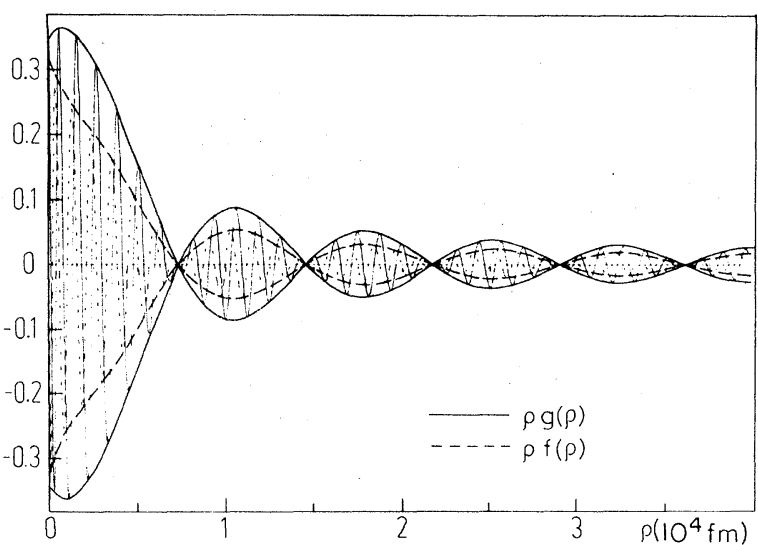

FIG. 3. A wave packet with a mean energy $E=2.15 m_{0} c^{2}$ and a width $\Delta E=0.30 m_{0} c^{2}$.

\section{B. The modified coupled-channel equations}

We now investigate the consequences arising from the use of two basis systems for bound and continuum electrons on the structure of the coupled-channel equations.

We begin by expressing the Hamiltonian (as far as continuum electrons are concerned) in terms of the new coordinates $(\vec{\rho}, \vec{R})$. Neglecting the interaction of the continuum electrons with the bound ones and the recoil effect of the continuum electrons on the nuclei,

$$
\left[g^{2}(R) / 2 \mu+1 / 2 M_{N}\right]\left(\sum_{K+1}^{z^{\prime}} \overrightarrow{\mathrm{p}}_{i}\right)^{2},
$$

we obtain in a way analogous to Sec. II.A:

$$
H\left(\overrightarrow{\mathrm{r}}_{1}, \cdots, \overrightarrow{\mathrm{r}}_{K}, \vec{\rho}_{K_{+1}}, \cdots, \vec{\rho}_{z^{\prime}} ; \overrightarrow{\mathrm{R}}\right)=\frac{p^{2}}{2 \mu}+V^{A B}(R)+H_{-\mathrm{mol}}^{K \text { el }}\left(\overrightarrow{\mathrm{r}}_{1}, \cdots, \overrightarrow{\mathrm{r}}_{K} ; \overrightarrow{\mathrm{R}}\right)+\sum_{i=K+1}^{z^{\prime}} \mathcal{K}\left(\vec{\rho}_{i} ; \overrightarrow{\mathrm{R}}\right) .
$$

Here $K$ is the number of bound electrons, and

$H_{\mathrm{mo1}}^{K \theta 1}\left(\overrightarrow{\mathrm{r}}_{1}, \cdots, \overrightarrow{\mathrm{r}}_{K} ; \overrightarrow{\mathrm{R}}\right)=\sum_{i=1}^{K}\left[\vec{\alpha}^{(i)} \cdot \overrightarrow{\mathrm{p}}_{i} c+\beta^{(i)} m_{0} c^{2}+V^{e A}\left(\overrightarrow{\mathrm{r}}_{i}, \overrightarrow{\mathrm{R}}\right)+V^{e B}\left(\overrightarrow{\mathrm{r}}_{i}, \overrightarrow{\mathrm{R}}\right)\right]+\frac{1}{2 M_{N}}\left(\sum_{i=1}^{K} \overrightarrow{\mathrm{p}}_{i}\right)^{2}+\frac{1}{2} \sum_{i \neq j}^{K} V^{e e}\left(\left|\overrightarrow{\mathrm{r}}_{i}-\overrightarrow{\mathrm{r}}_{j}\right|\right)$

is the complete molecular Hamiltonian for the $K$ bound electrons. The operators $\kappa\left(\rho_{i}, \overrightarrow{\mathrm{R}}\right)$ are given by

$$
\varkappa\left(\vec{\rho}_{i}, \overrightarrow{\mathrm{R}}\right)=\tilde{H}^{A}\left(\vec{\rho}_{i}, R\right)+g(R) \frac{m_{0}}{\mu} \vec{\alpha}^{(i)} \cdot \overrightarrow{\mathrm{P}} c+\left[V^{e A}\left(\left|\vec{\rho}_{i}+\left(g(R)+\frac{M_{B}}{M_{N}}\right) \overrightarrow{\mathrm{R}}\right|\right)+V^{e B}\left(\left|\vec{\rho}_{i}+\left(g(R)-\frac{M_{A}}{M_{N}}\right) \overrightarrow{\mathrm{R}}\right|\right)-\tilde{V}(\rho)\right] .
$$

They represent the molecular Hamiltonian for the continuum electrons (without recoil and electron-electron interaction), expressed in terms of the quasiatomic $\tilde{H}^{A}$ and correction terms. Thus, besides the correction potential $W=V^{e A}+V^{e B}-\tilde{V}$, there occur additional couplings by the relative momentum $\overrightarrow{\mathrm{P}}$ :

$$
g(R)\left(m_{0} / \mu\right) \vec{\alpha} \cdot \overrightarrow{\mathrm{P}} c,
$$

which are similar to the operator $(1 / 2 \mu)\{\overrightarrow{\mathrm{A}}, \overrightarrow{\mathrm{P}}\}$ from Section II D. In fact, considering $(1 / 2 \mu)\{\overrightarrow{\mathrm{A}}, \overrightarrow{\mathrm{P}}\}$ for a single electron, both terms become equal (in order $m / \mu$ ) for $R \rightarrow \infty$, according to Eq. (2.44). Therefore, we already know how to calculate the couplings due to $g(R)\left(m_{0} / \mu\right) \vec{\alpha} \cdot \overrightarrow{\mathbf{p}} c$, and no new problems arise from this term.

Because of the asymptotic identity

$$
\left(\frac{P^{2}}{2 \mu}+\frac{1}{2 \mu}\{\overrightarrow{\mathrm{A}}, \overrightarrow{\mathrm{P}}\}\right) \tilde{\varphi}_{\omega}^{A}\left(\vec{\rho}_{i}, R\right) \underset{R \rightarrow \infty}{\longrightarrow} \frac{P_{A B}^{2}}{2 \mu_{A B}} \tilde{\varphi}_{\omega}^{A}\left(\overrightarrow{\mathrm{r}}_{A}^{*}\right)=0,
$$

this term asymptotically cancels the spurious electronic excitations due to relative motion without a 
translation operator being necessary. This reflects the fact that $\tilde{\varphi}_{\omega}^{A}$ was constructed as to become an atomic state for $R \rightarrow \infty$.

We now make the following ansatz for the wavefunction:

$$
\Psi\left(\overrightarrow{\mathbf{r}}_{1}, \ldots, \overrightarrow{\mathbf{r}}_{K}, \vec{\rho}_{K+1}, \ldots, \vec{\rho}_{z} ; \overrightarrow{\mathrm{R}}\right)=\sum_{(m)} \hat{T}\left(\overrightarrow{\mathrm{X}}^{K}\right) \chi_{(m)}(\overrightarrow{\mathrm{R}}) \dot{\varphi}_{(m)}\left(\overrightarrow{\mathrm{r}}_{1}, \ldots, \overrightarrow{\mathrm{r}}_{K}, \vec{\rho}_{K+1}, \ldots, \vec{\rho}_{\mathbf{z}^{\prime}} ; \overrightarrow{\mathrm{R}}\right),
$$

where

$$
\begin{aligned}
& (m)=\left(m_{K} ; \omega_{K+1}, \ldots, \omega_{z^{\prime}}\right), \\
& \varphi_{(m)}\left(\overrightarrow{\mathrm{r}}_{0} ; \rho ; \overrightarrow{\mathrm{R}}\right)=Q\left[\varphi_{m_{K}}\left(\overrightarrow{\mathrm{r}}_{1}, \ldots, \overrightarrow{\mathrm{r}}_{K} ; R\right) \tilde{\varphi}_{\omega_{K+1}}^{A}\left(\overrightarrow{\mathrm{p}}_{K+1}, R\right) \ldots \tilde{\varphi}_{\omega_{z^{\prime}}}^{A}\left(\overrightarrow{\mathrm{p}}_{Z^{\prime}}, R\right)\right], \\
& H_{\operatorname{mol}}^{K e 1}\left(\overrightarrow{\mathrm{r}}_{1}, \ldots, \overrightarrow{\mathrm{r}}_{K} ; \overrightarrow{\mathrm{R}}\right) \varphi_{m_{K}}\left(\overrightarrow{\mathrm{r}}_{1}, \ldots, \overrightarrow{\mathrm{r}}_{K} ; R\right)=\epsilon_{m_{K}}(R) \varphi_{m_{K}}\left(\overrightarrow{\mathrm{r}}_{1}, \ldots, \overrightarrow{\mathrm{r}}_{K} ; R\right), \\
& \tilde{H}^{A}\left(\overrightarrow{\mathrm{p}}_{i}, R\right) \tilde{\varphi}_{\omega_{i}}^{A}\left(\overrightarrow{\mathrm{p}}_{i}, R\right)=\hbar \omega_{i} \tilde{\varphi}_{\omega_{i}}^{A}\left(\overrightarrow{\mathrm{p}}_{i}, R\right)\left(\left|\hbar \omega_{i}\right| \geqslant m_{0} c^{2}, i=K+1 \ldots, Z^{\prime}\right), \\
& \overrightarrow{\mathrm{X}}^{K}=\frac{m}{\mu} \sum_{i=1}^{K} \overrightarrow{\mathbf{s}}_{i} .
\end{aligned}
$$

Hence for all bound electrons translation effects are included by the translation operator $\hat{T}\left(\overrightarrow{\mathrm{X}}^{K}\right)$, whereas the continuum electrons have no effect on the scattering coordinate.

We now insert (4.14) and (4.18) into the Schrödinger equation $H \Psi=E \Psi$ and project out the electronic wave function and the translation operator. Our choice for $\overrightarrow{\mathrm{X}}^{K}$ has the consequence that, when transforming the Hamiltonian with $\hat{T}\left(\overrightarrow{\mathrm{X}}^{K}\right)$, only $H_{\text {mol }}^{K e 1}$ contributes to the operator $\overrightarrow{\mathrm{A}}$ :

$$
\overrightarrow{\mathrm{A}}^{K}=\frac{i}{\hbar} \mu\left[H_{\mathrm{mol}}^{K e 1}, \overrightarrow{\mathrm{X}}^{K}\right]=m \sum_{i=1}^{K} \frac{f\left(\overrightarrow{\mathrm{r}}_{i}, \overrightarrow{\mathrm{R}}\right)+\lambda}{2} \vec{\alpha}^{(i)} c \text {. }
$$

Thus we obtain (making the same approximations as in Sec. II D:

$$
\sum_{(K)}\left\langle\varphi_{(m)}\left|\frac{P^{2}}{2 \mu}+V^{A B}(R)+H_{\mathrm{mol}}^{K \theta 1}+\frac{1}{2 \mu}\left\{\overrightarrow{\mathrm{A}}^{K}, \overrightarrow{\mathrm{P}}\right\}+\sum_{i=K+1}^{z^{\prime}} \mathcal{x}\left(\overrightarrow{\mathrm{p}}_{i}, \overrightarrow{\mathrm{R}}\right)+\Delta-E\right| \varphi_{(n)}\right\rangle \chi_{(n)}(\overrightarrow{\mathrm{R}})=0,
$$

where

$$
\Delta=V\left(\overrightarrow{\mathrm{R}}-\overrightarrow{\mathrm{X}}^{K}\right)-V(\overrightarrow{\mathrm{R}})
$$

where $V$ is the sum of all interactions and the volume element in $\langle(m) \mid(n)\rangle$ is $d^{3} \gamma_{1} \ldots d^{3} r_{K} d^{3} \rho_{K+1} \ldots d^{3} \rho_{Z^{\prime}}$. Owing to the use of two different basic sets, two different configurations $|(n)\rangle,|(m)\rangle$ will in general not be orthogonal. Using

$$
\begin{aligned}
& \left\langle(m)\left|H_{\mathrm{mol}}^{K e 1}\right|(n)\right\rangle=\epsilon_{n_{K}}\langle(m) \mid(n)\rangle \\
& \left\langle(m)\left|\sum_{i=K+1}^{z^{\prime}} \kappa_{i}\right|(n)\right\rangle \chi_{(n)}=\left(\sum_{i=K+1}^{Z^{\prime}} \hbar \omega_{i}\right)\langle(m) \mid(n)\rangle \chi_{(n)}+g(R) \frac{m_{0}}{\mu}\left\langle(m)\left|\sum_{i=K+1}^{z \prime} \vec{\alpha}^{(i)} \cdot \overrightarrow{\mathrm{P}} c\right|(n)\right\rangle \chi_{(n)}+\left\langle(m)\left|\sum_{i=K+1}^{z^{\prime}} W_{i}\right|(n)\right\rangle \chi_{(K)}, \\
& \left\langle(m)\left|V^{A B}\right|(n)\right\rangle=V^{A B}(R)\langle(m) \mid(n)\rangle \\
& \epsilon_{(n)}(R)=\epsilon_{n_{K}}(R)+\sum_{i=K+1}^{Z^{\prime}} \hbar \omega_{i}
\end{aligned}
$$

Equation (4.21) becomes

$$
\begin{aligned}
\sum_{(n)} & {\left[\frac{1}{2 \mu}(\overrightarrow{\mathrm{P}} \cdot \underline{1}+\underline{\overrightarrow{\mathrm{P}}}+\underline{\overrightarrow{\mathrm{A}}})_{(m)(n)}^{2}+\left[V^{A B}(R)+\epsilon_{(n)}(R)-E\right]\langle(m) \mid(n)\rangle\right.} \\
& \left.+\left(\underline{\Delta}-\frac{1}{2 \mu} \overrightarrow{\mathrm{A}} \cdot \underline{\overrightarrow{\mathrm{A}}}\right)_{(m)(n)}+\left(\sum_{i=K^{\prime}+1}^{\mathbf{z}^{0}} W\left(\vec{\rho}_{i}, \overrightarrow{\mathrm{R}}\right)\right)_{(m)(n)}\right] \chi_{(n)}(\overrightarrow{\mathrm{R}})=0 .
\end{aligned}
$$

Here we put together the $\overrightarrow{\mathrm{A}}$-like terms:

$$
\overrightarrow{\mathrm{A}}_{(m)(n)} \equiv \overrightarrow{\mathrm{A}}_{(m)(n)}^{K}+g(R) m_{0}\left\langle(m)\left|\sum_{i=K+1}^{z^{\prime}} \vec{\alpha}^{(i)} c\right|(n)\right\rangle=\left\langle(m)\left|m \sum_{i=1}^{K} \frac{f\left(\overrightarrow{\mathrm{r}}_{i}, \overrightarrow{\mathrm{R}}\right)+\lambda}{2} \vec{\alpha}^{(i)} c+g(R) m_{0} \sum_{i=K+1}^{z^{\prime}} \vec{\alpha}^{(i)} c\right|(n)\right\rangle .
$$

Thus we have the following additional couplings (compared to Sec. II.D): 


$$
\left(V^{A B}+\epsilon_{(n)}-E\right)\left(\langle(m) \mid(n)\rangle-\dot{\delta}_{(m)(n)}\right)
$$

(due to nonorthogonality) and

$$
\left(\sum_{i=K+1}^{Z^{\prime}} W\left(\vec{\rho}_{i}, \overrightarrow{\mathrm{R}}\right)\right)_{(m)(n)}
$$

(due to the correction potential). The further steps (transformation to rotating coordinates and semiclassical approximation) are completely analogous to Sec. III, and we may directly write down the modified coupled-channel equations. To this end we define the modified radial and rotational couplings:

$$
\begin{aligned}
& \left\langle(m)\left|H^{\mathrm{rad}}\right|(n)\right\rangle \equiv \dot{R}\left[\left\langle(m)\left|-i \hbar \partial_{R}\right|(n)\right\rangle+A_{(m)(n)}^{R}\right], \\
& \left\langle(m)\left|H^{\mathrm{rot}}\right|(n)\right\rangle=-\delta_{\Lambda_{(m)}, \Lambda_{(n)} \pm 1} \frac{\hbar K}{\mu R^{2}}\left[\left(J_{y^{\prime}}\right)_{(m)(n)}-R A_{(m)(n)}^{x^{\prime}}\right] .
\end{aligned}
$$

We find

$$
\begin{gathered}
\dot{a}_{(m)}(t)=-\frac{i}{\hbar} \sum_{(n)}\left(\left\langle(m)\left|H^{\mathrm{rad}}+H^{\mathrm{rot}}\right|(n)\right\rangle+\left[V^{A B}(R)+\epsilon_{(n)}(R)-E\right]\left[\langle(m) \mid(n)\rangle-\delta_{(m)(n)}\right]\right. \\
\left.+\left\langle(m)\left|\sum_{i=K+1}^{\boldsymbol{z}^{\prime}} W_{i}\right|(n)\right\rangle\right) a_{(n)}(t) \exp \left(-\frac{i}{\hbar} \int_{0}^{t}\left(\epsilon_{(n)}-\epsilon_{(m)}\right) d t^{\prime}\right) .
\end{gathered}
$$

The structure of these equations complicates a little bit if we expand $\Psi$ in terms of the wave packets (4.12) instead of using the $\tilde{\varphi}_{\omega}^{A}$. Defining in that case

$$
\epsilon_{(n)} \equiv \epsilon_{n_{K}}+\sum_{i=K+1}^{Z^{\prime}} E_{i} \equiv \epsilon_{n_{K}}+\sum_{i=K+1}^{Z^{\prime}}\left\langle\Psi_{E_{i}}\left|\tilde{H}_{i}^{A}\right| \Psi_{E_{i}}\right\rangle,
$$

we obtain instead of (4.29) the following additional coupling:

$$
\left[V^{A B}(R)+\epsilon_{n_{K}}(R)-E\right]\left(\langle(m) \mid(n)\rangle-\delta_{(m)(n)}\right)+\sum_{i=K+1}^{z^{\prime}}\left[\left\langle(m)\left|\tilde{H}_{i}^{A}\right|(n)\right\rangle-E_{i}{ }_{(m)(n)}\right] .
$$

Since for $(m)=(n)$,

$$
\langle(m) \mid(n)\rangle=\delta_{(m)(n)} ;\left\langle(m)\left|\tilde{H}_{i}^{A}\right|(n)\right\rangle=E_{i} \delta_{(m)(n)},
$$

the coupled-channel equations can be written as

$$
\begin{aligned}
\dot{a}_{(m)}(t)=-\frac{i}{\hbar} \sum_{(n) \neq(m)}\left(\left\langle(m)\left|H^{\mathrm{rad}}+H^{\mathrm{rot}}\right|(n)\right\rangle+\left(V^{A B}+\epsilon_{n_{K}}-E\right)\langle(m) \mid(n)\rangle+\sum_{i=K+1}^{Z^{\prime}}\left\langle(m)\left|W_{i}\right|(n)\right\rangle\right) \\
\times a_{(n)}(t) \exp \left(-\frac{i}{\hbar} \int_{0}^{t}\left(\epsilon_{(n)}-\epsilon_{(m)}\right) d t^{\prime}\right) \\
-\frac{i}{\hbar}\left(\left\langle(m)\left|H^{\mathrm{rad}}\right|(m)\right\rangle+\left\langle(m)\left|\sum_{i=K+1}^{z^{\prime}} W_{i}\right|(m)\right\rangle\right) a_{(m)}(t) .
\end{aligned}
$$

Here we made use of the fact that for the Weyl wave packets (4.12),

$$
\left\langle\Psi_{E^{\prime}}\left|\tilde{H}^{A}\left(\vec{\rho}_{i}\right)\right| \Psi_{E}\right\rangle=\frac{1}{\Delta \omega} \int_{E^{\prime}}^{E_{+}^{\prime}} d \omega^{\prime} \int_{E_{-}}^{E_{+}} d \omega \delta\left(\omega-\omega^{\prime}\right)=0 \text { for } E \neq E^{\prime} .
$$

The last term in (4.36) may be eliminated by defining

$$
\begin{aligned}
& b_{(m)}(t) \equiv a_{(m)}(t) \exp \left(\frac{i}{\hbar}\left\langle(m)\left|H^{\mathrm{rad}}+\sum_{i=K+1}^{Z^{\prime}} W_{i}\right|(m)\right\rangle\right), \\
& \int_{0}^{t} \epsilon_{(m)} d t^{\prime} \equiv \int_{0}^{t} \epsilon_{(m)} d t^{\prime}-\frac{i}{\hbar}\left\langle(m)\left|H^{\mathrm{rad}}+\sum_{i} W_{i}\right|(m)\right\rangle .
\end{aligned}
$$

Since $\left\langle(m)\left|H^{\mathrm{rad}}\right|(m)\right\rangle \underset{R \rightarrow \infty}{\longrightarrow} 0$ and, as will be shown in the Sec. IV C, also

$$
\left\langle(m)\left|\sum_{i=K+1}^{Z^{\prime}} W_{i}\right|(m)\right\rangle \underset{R \rightarrow \infty}{\rightarrow} 0,
$$

we have 


$$
a_{(m)}(t \rightarrow \infty)=b_{(m)}(t \rightarrow \infty) ; \quad\left|a_{(m)}(t)\right|^{2}=\left|b_{(m)}(t)\right|^{2} .
$$

The new amplitudes $b_{(m)}(t)$ fulfill the equations

$\dot{b}_{(m)}(t)=-\frac{i}{\hbar} \sum_{(n) \neq(m)}\left(\left\langle(m)\left|H^{\mathrm{rad}}+H^{\mathrm{rot}}\right|(n)\right\rangle+\left(V^{A B}+\epsilon_{n_{K}}-E\right)\langle(m) \mid(n)\rangle+\sum_{i=K+1}^{z^{0}}\left\langle(m)\left|W_{i}\right|(n)\right\rangle\right) b_{(n)}(t) e^{-i \tilde{\chi}_{(n)(m)}^{\prime}(t)}$,

where

$$
\tilde{\chi}_{(n) \times m)}(t)=\frac{1}{\hbar} \int_{0}^{t}\left(\tilde{\epsilon}_{(n)}-\tilde{\epsilon}_{(m)}\right) d t^{\prime}
$$

In the following section we will study the coupling matrix elements in (4.40) in detail.

$$
\begin{gathered}
\text { C. Investigation of the additional coupling matrix } \\
\text { elements }
\end{gathered}
$$

(i) We first note that the matrix elements of $g(R) m_{0} \sum_{i=K+1}^{z^{\prime}} \vec{\alpha}^{(i)} c$ can be given the same form as the matrix elements of the translation vector for the bound electrons:

$$
\begin{aligned}
g(R) m_{0} \sum_{i=K+1}^{z^{\prime}}\left\langle(m)\left|\vec{\alpha}^{(i)} c\right|(n)\right\rangle & =\frac{i}{\hbar} g(R) m_{0}\left\langle(m)\left(\sum_{j=K+1}^{z^{\prime}} \tilde{H}_{j}^{A}, \sum_{i=K+1}^{z^{\prime}} \vec{\rho}_{i}\right)(n)\right\rangle \\
& =\frac{i}{\hbar} \sum_{i=K+1}^{z^{\prime}}\left(E_{i_{(m)}}-E_{i_{(n)}}\right)\langle(m)|\overrightarrow{\mathrm{Y}}|(n)\rangle,
\end{aligned}
$$

where

$$
\overrightarrow{\mathrm{Y}}=\sum_{j=K+1}^{\mathbf{z}^{\prime}} \frac{m_{0}}{\mu} g(R) \vec{\rho}_{i}
$$

is the analogue to the translation vector $\overrightarrow{\mathrm{X}}^{K}$.

(ii) Now the matrix elements of the correction potential $W\left(\vec{\rho}_{i}, \vec{R}\right)$ will be shown to be finite and to vanish for $R \rightarrow \infty$. For small $R, W$ only contains multipole contributions with $l \geqslant 1$ [according to our construction of $\tilde{V}(\rho)]$. The matrix elements $\left\langle\tilde{\varphi}_{\omega}^{A}|W| \tilde{\varphi}_{\omega^{\prime}}^{A}\right\rangle$ are finite, and the multipole $l$ part can be estimated by

$$
W_{\omega \omega^{\prime}}^{l} \sim \int \tilde{\varphi}_{\omega}^{A \dagger}(\vec{\rho}, R) \frac{P_{l}(\cos \theta)}{\rho^{l+1}} \tilde{\varphi}_{\omega^{\prime}}^{A}(\vec{\rho}, R) d^{3} \rho=I\left(\mu \kappa ; \mu^{\prime} \kappa^{\prime} ; l\right) \int_{0}^{\infty}\left(g_{\omega K} g_{\omega \cdot K^{\prime}}+f_{\omega K} f_{\omega \prime k^{\prime}}\right) \frac{1}{\rho^{l+1}} \rho^{2} d \rho,
$$

where

$$
I\left(\mu \kappa ; \mu^{\prime} \kappa^{\prime} ; l\right)=\int \chi_{\kappa}^{+\mu} \chi_{\kappa^{\prime}}^{\mu^{\prime}} P_{l} d l=\int \chi_{\kappa}^{+\mu} \chi_{\kappa \kappa}^{\mu^{\prime},} P_{l} d \Omega
$$

Splitting the $\rho$ integral in a finite part $W_{\mathrm{fin}}$ from 0 to $\tilde{\rho}\left(p \tilde{\rho} \gg 1, p^{\prime} \tilde{\rho} \gg 1\right)$ and another part from $\tilde{\rho}$ to $\infty$, where the $f_{\omega k}, g_{\omega k}$ may be approximated by their asymptotics $(4.8)$, we find

$$
\begin{aligned}
W_{\omega \omega^{\prime}}^{l} \sim W_{\mathrm{fin}}\left(\mu \kappa ; \mu^{\prime} \kappa^{\prime} ; l ; \omega \omega^{\prime} ; \tilde{\rho}\right)+\int_{\tilde{\rho}}^{\infty} \rho^{-(l+1)}\left\{A\left(\omega, \omega^{\prime}\right) \cos \left[p \rho+\delta_{\kappa}(p, \rho)\right] \cos \left[p^{\prime} \rho+\delta_{\kappa}\left(p^{\prime}, \rho\right)\right]\right. \\
\left.+B\left(\omega, \omega^{\prime}\right) \sin \left[p \rho+\delta_{\kappa}(p, \rho)\right] \sin \left[p^{\prime} \rho+\delta_{\kappa}\left(p^{\prime}, \rho\right)\right]\right\} d \rho,
\end{aligned}
$$

where

$$
\begin{aligned}
& A\left(\omega, \omega^{\prime}\right)=\left(\frac{\left(\hbar \omega+m_{0} c^{2}\right)\left(\hbar \omega^{\prime}+m_{0} c^{2}\right)}{\pi^{2} c^{2} p p^{\prime}}\right)^{1 / 2}, \\
& B\left(\omega, \omega^{\prime}\right)=\left(\frac{\left(\hbar \omega-m_{0} c^{2}\right)\left(\hbar \omega^{\prime}+m_{0} c^{2}\right)}{\pi^{2} c^{2} p p^{\prime}}\right)^{1 / 2} .
\end{aligned}
$$

The second integral exists for $l \geqslant 1$, since it is bounded from above by $\int_{\tilde{\rho}}^{\infty} \rho^{-l-1} d \rho$. However, for $l=0$ it is undetermined (due to the logarithmic Coulomb phase). Therefore, it is important that $\tilde{V}(\rho)$ in the limit $R \rightarrow \infty$ correctly contain the long-range monopole part of the two-center potential.

For $R \rightarrow \infty$, on the other hand, $W(\vec{\rho}, \overrightarrow{\mathrm{R}})$ approaches $V^{{ }^{B}}\left(r_{B}\right)$ :

$$
W(\vec{\rho}, \overrightarrow{\mathrm{R}}) \underset{R \rightarrow \infty}{\longrightarrow}-\frac{Z_{B} e^{2}}{|\vec{\rho}-\overrightarrow{\mathrm{R}}|}=-Z_{B} e^{2} \begin{cases}\sum_{l} \frac{\rho^{l}}{R^{l+1}} P_{l}(\cos (\vec{\rho}, \overrightarrow{\mathrm{R}})), & \rho<R \\ \sum_{l} \frac{R^{l}}{\rho^{l+1}} P_{l}(\cos (\vec{\rho}, \overrightarrow{\mathrm{R}})), & R \leqslant \rho .\end{cases}
$$


Inserting this into the matrix element, one easily sees (in a manner analogous to the above reasoning) that the $l=0$ part does not exist, and the $l=1$ part does not vanish for $R \rightarrow \infty$, if one uses the $\tilde{\varphi}_{\omega}^{A}$. However, taking the wave packets $\Psi_{E}$ as a basis, we may avoid this problem. The asymptotics of the wave packets can be calculated; for the large component, for example, we get

$$
\left[\Psi_{E}(\rho, R, t)\right]_{1 \mathrm{c}} \underset{\rho \rightarrow \infty}{\longrightarrow} \frac{1}{(\Delta \omega)^{1 / 2}} \int_{E_{-}}^{E_{+}} \frac{\cos \left[p \rho+\delta_{\mathrm{K}}(p, \rho)\right]}{\rho} e^{-i \omega t} d \omega \chi_{\kappa}^{\mu} .
$$

For large $\rho$, the variation of the logarithmic phase $\delta_{k}$ within the interval $\left[E_{-}, E_{+}\right]$may be neglected with respect to the variation of $p \rho$. Thus a similar estimate as in Ref. 39 shows that

$$
\Psi_{E}(\vec{\rho}, R, t) \underset{\rho \rightarrow \infty}{\longrightarrow} \frac{A(\vec{\rho}, E, t)}{\rho\left[\rho-c t\left(1-m_{0}^{2} c^{4} / E^{2}\right)^{1 / 2}\right]},
$$

where $A(\vec{\rho}, E, t)$ is a spinor amplitude with absolute length $<1$. Thus, for a given time $t$, the wave packet decays like $1 / \rho^{2}$ for $\rho \rightarrow \infty$. As a function of time it spreads such that the amplitude decreases like $1 / t$. Now the second part of the matrix element of $W$

$$
W_{E E^{\prime}}=\frac{1}{\Delta \omega} \sum_{l}\left(\int_{0}^{R} \int_{0}^{4 \pi} \Psi_{E}^{\dagger} \frac{\rho^{l} P l}{R^{l+1}} \Psi_{E^{\prime}} \rho^{2} d \rho d \Omega+\int_{R}^{\infty} \int_{0}^{4 \pi} \Psi_{E}^{\dagger} \frac{R^{l} P_{l}}{\rho^{l+1}} \Psi_{E^{\prime}} \rho^{2} d \rho d \Omega\right)
$$

for $R \rightarrow \infty$ becomes

$$
\sim \sum_{l} R^{l} \int_{R}^{\infty} \frac{\tilde{A}_{l}\left(\rho, E, E^{\prime}, t\right)}{\rho^{4}} \frac{1}{\rho^{l+1}} \rho^{2} d \rho,
$$

with a function $\left|\tilde{A}_{l}\left(\rho, E, E^{\prime}, t\right)\right| \leqslant 1$. Hence, the multipole $l$ of the integral behaves like

$$
\leqslant R^{l} \int_{R}^{\infty} \rho^{-l-3} d \rho \sim R^{-2-l}
$$

The first integral in (4.49) may be estimated similarly. First consider the case $l=0$ :

$$
\begin{aligned}
\frac{1}{\Delta \omega} \int_{0}^{R} \Psi_{E}^{\dagger} \frac{P_{0}(\cos \theta)}{R} \Psi_{E^{\prime}} \rho^{2} d \rho d \Omega & \sim \frac{1}{R} \int_{0}^{R} \int_{0}^{4 \pi} \Psi_{E^{\prime}}^{\dagger} \Psi_{E^{\prime}} \rho^{2} d \rho d \Omega \\
& =\frac{1}{R}\left\langle\Psi_{E} \mid \Psi_{E^{\prime}}\right\rangle-\frac{1}{R} \int_{R}^{\infty} \int_{0}^{4 \pi} \Psi_{E}^{\dagger} \Psi_{E^{\prime}} \rho^{2} d \rho d \Omega .
\end{aligned}
$$

Because of $E^{\prime} \neq E$ the first term vanishes, and the second is $\sim R^{-2}$ according to Eqs. (4.51) and (4.52). Thus the monopole part of $W_{E E}$, vanishes like $1 / R^{2}$ for $R \rightarrow \infty$. The higher multipoles even decay more rapidly because of

$$
\frac{1}{\Delta \omega} \int_{0}^{R} \int_{0}^{4 \pi} \Psi_{E}^{\dagger} \frac{\rho^{l} P_{l}(\cos \theta)}{R^{l+1}} \Psi_{E^{\prime}} \rho^{2} d \rho d \Omega \leqslant \frac{1}{\Delta \omega} \frac{R^{l}}{R^{l+1}} \int_{0}^{R} \int_{0}^{4 \pi} \Psi_{E}^{\dagger} \Psi_{E^{\prime}} \rho^{2} d \rho d \Omega
$$

[since $\rho \leqslant R$ and $\left.\left|P_{l}(\cos \theta)\right| \leqslant 1\right]$.

Cons equently, we have proved that, using wave packets, the couplings by the additional potential $W$ vanish like $1 / R^{2}$ for $R \rightarrow \infty$.

(iii) Finally, we have to care about the overlap $\langle(m) \mid(n)\rangle((m) \neq(n))$, which in general is determined by the overlap $\left\langle\varphi_{n} \mid \tilde{\varphi}_{\omega}^{A}\right\rangle$ or $\left\langle\varphi_{n} \mid \Psi_{E}\right\rangle$, respectively, between continuum states and bound molecular states.

$\{\langle(m) \mid(n)\rangle \neq 0$ even if $|(m)\rangle$ and $|(n)\rangle$ contain the same numbers of bound and continuum electrons. This is due to antisymmetrization. Consider a two-electron system, let $\varphi, \varphi^{\prime}$ be two different bound one-electron states $\left(\left\langle\varphi \mid \varphi^{\prime}\right\rangle=0\right)$ and $\psi$ a one-electron continuum state $\left(\langle\varphi \mid \psi\rangle \neq 0,\left\langle\varphi^{\prime} \mid \psi\right\rangle \neq 0\right)$. The two-electron system is described by

$$
|(m)\rangle=(1 / \sqrt{2})[|\varphi(1)\rangle|\psi(2)\rangle-|\varphi(2)\rangle|\psi(1)\rangle]
$$

and

$$
\left|\left(m^{\prime}\right)\right\rangle=(1 / \sqrt{2})\left[\left|\varphi^{\prime}(1)\right\rangle|\psi(2)\rangle-\left|\varphi^{\prime}(2)\right\rangle|\psi(1)\rangle\right] .
$$

Consequently, $\left\langle\left(m^{\prime}\right) \mid(m)\right\rangle=-\left\langle\varphi^{\prime} \mid \psi\right\rangle\langle\psi \mid \varphi\rangle \neq 0$ does not vanish. $\}$

There are two possibilities for $\varphi_{n}$. For $R \rightarrow \infty$ the bound electron (let us assume for simplicity that there is only one) can belong to nucleus $A$ or nucleus $B$. In the first case we remember that for $R \rightarrow \infty$ (one electron!) 


$$
H_{\mathrm{mol}}^{\mathrm{e} 1} \rightarrow \tilde{H}^{A}-\frac{m_{0}}{\mu} \vec{\alpha} \cdot \overrightarrow{\mathrm{P}} c+V^{e B}
$$

For electrons around nucleus $A$ the influence of $V^{e B}$ vanishes for $R \rightarrow \infty$; hence, the bound states of $H_{\mathrm{mol}}^{\text {el }}$ become identical to the bound states of $\tilde{H}^{A}$ up to terms $\sim m / \mu$, stemming from $-\left(m_{0} / \mu\right) \vec{\alpha} \cdot \overrightarrow{\mathrm{P}} c$, and have to be traced to translation effects. Consequently, $\left\langle\varphi_{n} \mid \tilde{\varphi}_{\omega}^{A}\right\rangle$ and $\left\langle\varphi_{n} \mid \Psi_{E}\right\rangle$ are of order $m / \mu$. A more detailed analysis shows that

$$
\left\langle\tilde{\varphi}_{\omega}^{A} \mid \varphi_{n}\right\rangle=\frac{1}{\epsilon_{n}}\left\langle\tilde{\varphi}_{\omega}^{A}\left|H_{\mathrm{mol}}^{\mathrm{el}}\right| \varphi_{n}\right\rangle \underset{R \rightarrow \infty}{\longrightarrow} \frac{1}{\epsilon_{n}}\left\langle\tilde{\varphi}_{\omega}^{A}\left|\tilde{H}^{A}-\frac{m}{\mu} \vec{\alpha} \cdot \overrightarrow{\mathrm{P}} c\right| \varphi_{n}\right\rangle,
$$

whence

$$
\frac{\mu}{2} V_{\infty}^{2}\left\langle\tilde{\varphi}_{\omega}^{A} \mid \varphi_{n}\right\rangle \underset{R \rightarrow \infty}{\longrightarrow} \frac{\mu}{2} V_{\infty}^{2} \frac{1}{\hbar \omega-\epsilon_{n}}\left\langle\tilde{\varphi}_{\omega}^{A}\left|\frac{m}{\mu} \vec{\alpha} \cdot \overrightarrow{\mathrm{P}} c\right| \varphi_{n}\right\rangle .
$$

The matrix element on the right-hand side has the form $(1 / 2 \mu)(\vec{A} \cdot \vec{P})_{\omega n}$ and, according to Sec. III C is an order $m / \mu$ smaller than the radial coupling matrix element. Setting $h \omega-\epsilon_{n}$ approximately equal to the kinetic energy of the electron in the continuum state $\tilde{\varphi}_{\omega}^{A}, \simeq(m / 2) v^{2}$, we see that (4.55) is by a factor

$$
\frac{m}{\mu} \frac{\mu}{2} V_{\infty}^{2} \frac{1}{(m / 2) v^{2}}=\left(\frac{V_{\infty}}{v}\right)^{2}<1
$$

smaller than the radial coupling. The higher the energy of the continuum state and the smaller the relative velocity of the two nuclei, the better justified is the neglect of the asy mptotic overlap $\langle(m) \mid(n)\rangle$. Here essentially enters the nonrelativistic behavior of the nuclei. In addition, it will not be possible to treat continuum electrons with very low kinetic energy by this formalism. To get an idea of the order of magnitude for (4.56), assume $V_{\infty}=0.1 c$; for $\hbar \omega=m_{0} c^{2}+50 \mathrm{keV}$ we have $\left(V_{\infty} / v\right)^{2}$ $=0.05$, for $\hbar \omega=m_{0} c^{2}+100 \mathrm{keV}$ we have $\left(V_{\infty} / v\right)^{2}$. $=0.02$.

If $\varphi_{n}$ asymptotically becomes bound to nucleus $B$, there occurs additionally an overlap with the potential $V^{e B},\left\langle\tilde{\varphi}_{\omega}^{A}\left|V^{e B}\right| \varphi_{n}\right\rangle$ or $\left\langle\Psi_{E}\left|V^{e B}\right| \varphi_{n}\right\rangle$, respectively. This matrix element goes like $1 / R$ or $1 / R^{2}$, respectively, if $R$ becomes larger than the effective radius of the bound state $\varphi_{n}$. For very large $R$ thus again a term like (4.55) or (4.56) determines the asymptotic smallness of the coupling matrix elements.

Summarizing the results of this paragraph, all couplings occurring in the modified coupled-channel equation (4.40) can be calculated. They are finite for all times and vanish for $t \rightarrow \infty$ up to small contributions of order $\left(V_{\infty} / v\right)^{2}$ with respect to the radial coupling, as long as the states involved do not contain very low energetic continuum electrons. Hence this formulation allows for a calculation of the high-energy part of the $\delta$ electron and positron spectra, which is as exact as the treatment of the bound states. (Of course, errors in the description of low-energy continuum states may influence the high-energy spectra via multistep processes. Existing calculations in the monopole approximation ${ }^{5,15}$ suggest, how ever, that discretizing the continuum in $50-\mathrm{keV}$ steps does not influence the spectra. Thus it is relatively unimportant how one treats the continuum electrons with kinetic energy $<50 \mathrm{keV}$. Whether this remains true for the angular distribution of the continuum spectra has to be checked in actual calculations. If so, it means that our continuum basis constructed with the help of wave packets with an energy spacing of order $50 \mathrm{keV}$ is in fact "complete enough" for the description of continuum excitations.) For the low-energy part (kinetic energy less than $50 \mathrm{keV}$ ) of the continuum, errors in the order of the asymptotic (uncorrected) radial coupling occur.

\section{The calculation of electron spectra}

Let $a_{m n}^{K}(t)$ be the amplitude for a transition of the electronic shell from state $m$ to state $n$ in a collision with total angular momentum $K$ (impact parameter $b=h K / P_{\infty}$ ). The cross section for the transition $m \rightarrow n$ is given by

$$
\frac{(d \sigma)_{m n}}{d \Omega_{\text {nuc }}}=\left|a_{m n}^{K}(\infty)\right|_{K=a \cot \theta / 2}^{2}\left(\frac{d \sigma}{d \Omega_{\text {nuc }}}\right)_{\text {Ruth }},
$$

where $d \Omega_{\text {nuc }}$ is the angular volume element for the scattered nuclei.

The probability for creating one continuum electron in a collision with impact parameter $b$ is given by 
the sum of all transition probabilities into states $n$ which contain one continuum electron:

$$
\left(\frac{d \sigma}{d \Omega_{\mathrm{nuc}}}\right)_{1 \sigma \text { el }}=\sum_{n \ni 1 \sigma \mathrm{el}}\left|a_{m n}^{K}(\infty)\right|_{K=a \cot \theta / 2}^{2}\left(\frac{d \sigma}{d \Omega_{\mathrm{nuc}}}\right)_{\mathrm{Ruth}} d n,
$$

or, equivalently, expressed in terms of the impact parameter $b$ :

$$
[P(b)]_{16 \mathrm{e} 1}=\sum_{n \ni 16 \mathrm{e} 1}^{f}\left|a_{m n}^{K}(\infty)\right|_{\hbar K=b P_{\infty}}^{2} P(b)_{\mathrm{Ruth}} d n .
$$

The total cross section for creating one $\delta$ electron is

$$
\sigma_{16 \mathrm{el}}=2 \pi \int_{0}^{\infty} P(b)_{\mathrm{Ruth}} b d b\left(\sum_{n \ni 1 \delta \mathrm{e} 1}\left|a_{m n}^{K}(\infty)\right|_{\hbar K=b P_{\infty}}^{2} d n\right) .
$$

There are corresponding expressions for the creation of two or more continuum electrons (or positrons). The total number of 0 electrons is given by

$$
N(\delta \mathrm{el}) \sim \sum_{k} k \sigma_{k \delta \mathrm{el}} .
$$

In order to study the differential cross section, let us assume one-electron systems for simplicity of presentation. The energy spectrum of the $\delta$ electrons is obtained from (4.60) by differentiating with respect to the electron energy:

$$
\frac{d \sigma}{d E_{e}}=2 \pi \int_{0}^{\infty} P(b)_{\mathrm{Ruth}} b d b\left(\sum_{\left(E_{i}\right)}\left|a_{\left(E_{i}\right)}^{K}(\infty)\right|_{\hbar K=b P_{\infty}}^{2} j\left(E_{e}-E_{i}\right)\right) .
$$

Here we assumed that for the continuum states wave packets with mean energies $E_{i}$ have been used, for which

$$
d n=\sum_{\left(E_{i}\right)} \delta\left(E_{E}-E_{i}\right) d E_{e} .
$$

This line spectrum may be smeared out by writing

$$
\frac{d \sigma}{d E_{e}}=2 \pi \int_{0}^{\infty}\left(\sum_{\left(E_{i}\right)}\left|a_{m\left(E_{i}\right)}^{K}(\infty)\right|_{\hbar K=b P_{\infty}}^{2} f\left(E_{e}, E_{i}\right)\right) P(b)_{\mathrm{Ruth}} b d b,
$$

where $f\left(E_{e}, E_{i}\right)$ is a smeared-out version of the $\delta$ function $\delta\left(E_{e}-E_{i}\right)$. In order to derive the angular distribution of the spectrum, note that the angular distribution of a continuum state with quantum numbers $\left(E_{i}\right),\left(\Psi_{E_{i}}\right)_{k}^{\mu}$ is given by the spinors $\chi_{ \pm \kappa}^{\mu}\left(\vartheta_{A}, \varphi_{A}\right)$; here $\vartheta_{A}, \varphi_{A}$ are the electron angles in the coordinate system specified by $\tilde{H}^{A}$, i.e., for $t \rightarrow \infty$ in the coordinate system of nucleus $A$. Because of the orthogonality of the $\chi_{\kappa}^{\mu}$ we obtain ${ }^{38,40}$

$$
\frac{d \sigma}{d E_{e} d \Omega_{A} d b}=2 \pi b P(b)_{\mathrm{Ruth}} \sum_{E_{i}} f\left(E_{i}, E_{e}\right) \sum_{\mu, \kappa} \chi_{\kappa}^{\dagger \mu}\left(\Omega_{A}\right) \chi_{\kappa}^{\mu}\left(\Omega_{A}\right)\left|\left[a_{m E_{i}}^{K}(\infty)\right]_{\kappa}^{\mu}\right|_{\hbar K=b P_{\infty}}^{2} .
$$

We stated the differential cross section with respect to the impact parameter, since the orientation of the system, i.e., the asymptotic internuclear, axis depends on $b$, and the electron angles $\vartheta_{A}, \varphi_{A}$ are defined with respect to this axis. Hence $(4.64)$ gives a prediction for the angular distribution of $\delta$ electrons with energy $E_{e}$ measured in coincidence with the scattered projectile. If we want to calculate the angular distribution of the $b$-integrated spectrum, we first have to transform the angular characteristics $\left(\chi_{\kappa}^{\dagger \mu} \chi_{k}^{\mu}\right)\left(\Omega_{A}\right)$ to $b$-independent coordinates $\Omega_{e}$ and then integrate over $b$.

This transformation is split up into two steps:

(1) transformation from the system fixed at nucleus $A$ (i.e., moving with respect to CMN) to a parallel system resting with respect to CMN:

$$
\left(\vartheta_{A}, \varphi_{A}\right) \rightarrow\left(\vartheta_{A}^{\prime}, \varphi_{A}^{\prime}\right)
$$

(2) rotation to the nonrotating (i.e., space-fixed) CMN system:

$$
\left(\vartheta_{A}^{\prime}, \varphi_{A}^{\prime}\right) \rightarrow\left(\vartheta_{e}, \varphi_{e}\right)
$$

The definition of the angles is shown in Fig. 4. $Z$ is the $Z$ axis of the nonrotating CMN system (i.e., the beam axis); $\left(\vartheta_{e}, \varphi_{e}\right)$ are defined with respect to this axis. $Z^{\prime}$ is the $z$ axis of the rotating CMN system for $t \rightarrow \infty$ and also of the system fixed at nucleus $A . \quad\left(\vartheta_{A}, \varphi_{A}\right)$ and $\left(\vartheta_{A}^{\prime}, \varphi_{A}^{\prime}\right)$ are defined with respect to that axis. All $\varphi$ angles are zero in the scattering plane. 


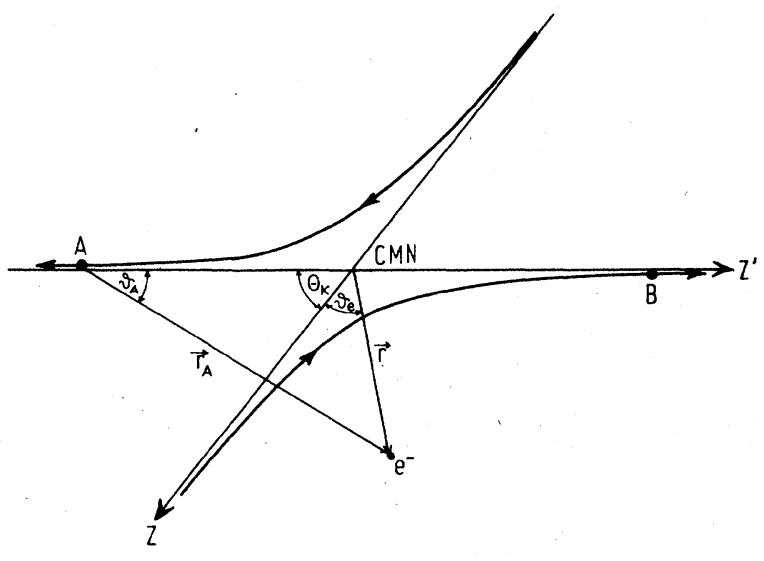

FIG. 4. Definition of the angles of the different coordinate systems used.

(1) The transformation (1) depends upon the energy of the electron. An electron with energy $E_{e}$ in the system moving with $A$ [i.e., which is emitted with momentum $\left.p c=\left(E_{a}^{2}-m_{0}^{2} c^{4}\right)^{1 / 2}\right]$ has in the nonmoving system the momentum $\overrightarrow{\mathrm{p}}+\Delta \overrightarrow{\mathrm{p}}$ $=\overrightarrow{\mathrm{p}}-m_{0}\left(M_{B} / M_{N}\right) \overrightarrow{\mathrm{R}}$ and, correspondingly, the energy

$$
E_{e}^{\prime}=\left[E_{e}^{2}+2 p \Delta p c^{2} \cos \vartheta_{A}+(\Delta p)^{2} c^{2}\right]^{1 / 2} ;
$$

whence

$$
d E_{e}^{\prime}=\frac{E_{e}}{E_{e}^{\prime}}\left(1+\frac{2 \Delta p}{p} \cos \vartheta_{A}\right) d E_{e}
$$

The order of magnitude for the change in momentum is $\Delta p c \sim 25 \mathrm{keV}\left(\dot{R}=0.1 c ; M_{N}=2 M_{B}\right)$, and the change in energy $\Delta E_{\lessgtr} 0.05 m_{0} c^{2} p c / E_{\text {( }}$ (i.e., also up to $\pm 25 \mathrm{keV}$ ).

In addition the angular volume element $\Omega_{A}$ is changed-an electron emitted in $A$ with angles $\vartheta_{A}, \varphi_{A}$ has in the nonmoving system the angles $\vartheta_{A}^{\prime}, \varphi_{A}^{\prime}=\varphi_{A} . \quad \vartheta_{A}^{\prime}$ may be derived from Fig. 5 by

$$
\begin{aligned}
\cos \vartheta_{A}^{\prime} & =\frac{(\vec{p}+\Delta \vec{p}) \cdot \Delta \vec{p}}{|\vec{p}+\Delta \vec{p}| \cdot \Delta p}=\frac{p \cos \vartheta_{A}+\Delta p}{|\vec{p}+\Delta \vec{p}|} \\
& =\frac{p \cos \vartheta_{A}+\Delta p}{\left[p^{2}+(\Delta p)^{2}+2 p \Delta p \cos \vartheta_{A}\right]^{1 / 2}} .
\end{aligned}
$$

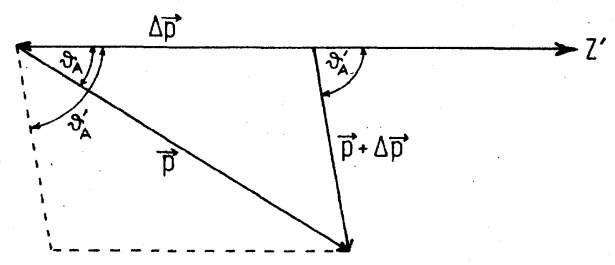

FIG. 5., Transformation of the angular volume element $d \Omega_{A} \rightarrow d \Omega_{A}^{\prime}$.
Hence

$$
d \cos \vartheta_{A}^{\prime}=d \cos \vartheta_{A}\left(\frac{p}{|\vec{p}+\Delta \vec{p}|}-\frac{p^{2} \Delta p \cos \vartheta_{A}}{|\vec{p}+\Delta \vec{p}|^{3}}\right) .
$$

As a result we obtain in the nonmoving system

$$
\begin{aligned}
\frac{d \sigma}{d E_{e}^{\prime} d \Omega_{A}^{\prime} d b}= & \frac{d \sigma}{d E_{e} d \Omega_{A} d b} \\
& \times \frac{E_{e}^{\prime}}{E_{e}}\left(1+\frac{2 \Delta p}{p} \cos \vartheta_{A}\right)^{-1} \\
& \times\left(\frac{p}{|\overrightarrow{\mathrm{p}}+\Delta \overrightarrow{\mathrm{p}}|}-\frac{p^{2} \Delta p \cos \vartheta_{A}}{|\overrightarrow{\mathrm{p}}+\Delta \overrightarrow{\mathrm{p}}|^{3}}\right)^{-1} .
\end{aligned}
$$

(2) In the second step we translate from the angles $\left(\vartheta_{A}^{\prime}, \varphi_{A}^{\prime}\right)$ defined with respect to the $Z^{\prime}$ axis to angles $\left(\vartheta_{e}, \varphi_{e}\right)$ defined with respect to the $Z$ axis. According to Fig. 6 and the cosine theorem of spherical trigonometry we have

$$
\cos \vartheta_{A}^{\prime}=-\cos \theta_{n u c} \cos \vartheta_{e}+\sin \theta_{\text {nuc }} \sin \vartheta_{e} \sin \varphi_{e},
$$

whereas the sine theorem yields

$$
\sin \varphi_{A}^{\prime}=\frac{\sin \vartheta_{Q}}{\sin \vartheta_{A}^{\prime}} \sin \varphi_{e} .
$$

This is a pure rotation so that $d \Omega_{e}=d \Omega_{\Lambda^{\prime}}$. Thus we get $d \sigma / d E_{s}^{\prime} d \Omega_{e} d b$ by inserting (4.70) and (4.71) into $d \sigma / d E_{e}^{\prime} d \Omega_{A}^{\prime} d b$. Substituting $\theta_{\text {suc }}$ by the impact parameter $b$ allows one to integrate over $b$ (numerically) in order to obtain the double differential cross section $d \sigma / d E_{e}^{\prime} d \Omega_{e}$, i.e., the angular distribution of electrons with energy $E_{e^{\prime}}$ in the nonrotating CMN system.

It is obvious that $d \sigma / d E_{e}^{\prime} d \Omega_{e}$ contains much less information than the coincidence spectrum $d \sigma / d E_{e}^{\prime} d \Omega_{e} d b$, where the scattered projectile is measured, too, and thus the $Z^{\prime}$ axis is specified. An anisotropy with respect to the $Z^{\prime}$ axis thus may easily be smeared out by integrating over the impact parameter. Consequently, isotr opy of $d \sigma / d E_{e}^{\prime} d \Omega_{e}$ (see, e.g., Refs. 36,37 ) does not tell us much about the isotropy of the coincidence spectrum $d \sigma / d E_{e}^{\prime} d \Omega_{e} d b$.

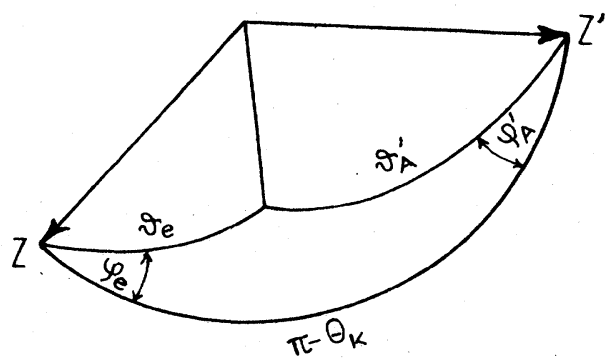

FIG. 6. Rotation from $\left(\vartheta_{A}^{\prime}, \varphi_{A}^{\prime}\right)$ to $\left(\vartheta_{e}, \varphi_{e}\right)$. 
The formalism developed in this section in order to treat continuum electrons (and positrons) for the first time provides a concept for the determination of angular distributions of $\delta$ electrons and positrons produced in heavy-ion collisions. Thus theory must be able to reproduce recently published data (Refs. 36,37 ) for $d \sigma / d E_{e}^{\prime} d \Omega_{e}$ (which show an anisotropy less than $15 \%)$; additionally, it should yield predictions for angular distributions of coincidence spectra. The necessary numerical calculations still have to be performed. Here we confine ourselves to the presentation of the formalism.

\section{THE SWITCHING FUNCTION}

Finding a suitable switching function $f(\overrightarrow{\mathrm{r}}, \overrightarrow{\mathrm{R}})$ has been the topic of many papers. ${ }^{18,41-43,51,52}$ As already mentioned in Sec. II C, the switching function is not fixed except for the boundary conditions at $R=0$ and $R \rightarrow \infty$. All choices of $f(\overrightarrow{\mathrm{r}}, \overrightarrow{\mathrm{R}})$ fulfilling these conditions are formally equivalent (as long as a complete set of electron wave functions is used), and therefore many different forms for the switching function have appeared in the literature. Usually $f(\vec{r}, \overrightarrow{\mathrm{R}})$ is specified by applying the criterion that a proper choice of the switching function should simplify the numerical solution of the coupled-channel equations. Some authors use switching functions with free parameters, which then are optimized as to minimize the coupling matrix elements within a limited number of electron states. ${ }^{41,43}$ It was shown that a proper choice of the switching function may considerably reduce the number of necessary basis states for the solution of the coupled-channel equations for the electron amplitudes. ${ }^{41,43,44}$ However, often the switching functions finally obtained depend upon the states taken into account ${ }^{41,43,51,52}$ and hence are not universal (thus violating the first of the criteria established by Schneiderman and Russek, ${ }^{18}$ or they are given only implicitly and can only be used with considerable numerical difficulties. ${ }^{51,52}$ Parameter-free forms usually are constructed only as to satisfy the asymptotic conditions and to interpolate in between in some meaningful manner, but lack a physical picture behind them. ${ }^{42}$ One exception, however, is the $f$ function given in Ref. 44, which will be discussed below and which we will finally employ.

In this section we will discuss two models for the switching function. The first one contains one parameter which will be determined by an optimization procedure to be discussed below, and thus falls into the class of trial switching functions used in Refs. 41 and 43. However, it turns out not to be universal, and therefore it will finally be rejected. The second one ${ }^{44}$ is derived from first principles using the physical picture standing behind the translation-factor idea. We will show that in the region where we can test the switching function, both forms, i.e., the optimized one-parameter form and the parameter-free form, yield very similar results. The reduction of the coupling matrix elements by application of the switching function in the investigated $R$ range is in the same range as achieved by other authors. ${ }^{41,43}$ This to our mind strongly favors the switching function we used. However, we do not consider this as a proof that our switching function is the only correct one, or the best one. More work on this field is still to be done.

In order to arrive at a sensible form for the switching function, let us remember the original idea of electron translation factors, i.e., to cancel the spurious asymptotic couplings resulting from the neglection of translation effects. We demand that $f(\overrightarrow{\mathrm{r}}, \overrightarrow{\mathrm{R}})$ be chosen in such a way that the translation matrix elements $\overrightarrow{\mathrm{A}}_{m n}$ always just cancel the spurious part of the dynamical coupling $\overrightarrow{\mathrm{P}}_{m n}$. This spurious part will vanish in the molecular limit where $\overrightarrow{\mathrm{P}}_{m n}$ contains no spurious contributions. For $R \rightarrow \infty$ it will become maximal (e.g., the $P_{m n}^{R}$ couplings are completely spurious in this limit). In the intermediate region the translational effect will be determined by the extent to which the electrons feel attached to nucleus $A$ or $B$.

We tested the following two models for the switching function.

(1) Assuming that the degree of attachment essentially is given by the ratio of electron radius to nuclear distance (i.e., the smaller, for example $r_{A} / R$ becomes, the more the electron belongs to nucleus $A$ ), one can make the following one-parameter ansatz fulfilling the boundary conditions of Sec. II C:

$$
\frac{\lambda+f(\overrightarrow{\mathrm{r}}, \overrightarrow{\mathrm{R}})}{2}=\tanh \left[g R\left(\frac{1}{r_{B}}-\frac{1}{r_{A}}\right)\right] \times \begin{cases}\frac{1+\lambda}{2}, & r_{B}<r_{A} \\ \frac{1-\lambda}{2}, & r_{B} \geqslant r_{A}\end{cases}
$$

where $g$ physically means the critical ratio $R / r_{A}$ or $R / r_{B}$, respectively, where the "molecular" electron becomes an "atomic" electron. ' $g$ can be determined by an optimization procedure; as an example, we may postulate that the sum of the corrected matrix elements for all (radial and rotational) couplings to a given state $m$ should become minimal:

$$
\frac{1}{N} \sum_{n=1}^{N} \frac{\left[\overrightarrow{\mathrm{P}}_{m n}+\overrightarrow{\mathrm{A}}_{m n}(g)\right]^{2}}{\left(\overrightarrow{\mathrm{P}}_{m n}\right)^{2}} \rightarrow \min .
$$


If the ansatz (5.1) is good, then the resulting $g_{\text {opt }}$ will be universal. In general, however, $g_{\text {opt }}$ will depend upon the nuclear distance $R$ and the state $m$ considered:

$$
g_{\text {opt }}=g_{\text {opt }}(R, m) \text {. }
$$

We investigated this behavior numerically; the results of this ad hoc minimization procedure will be presented below and compared to another, parameter-free choice for the switching function which can be obtained from first principles via the following physical consideration ${ }^{44}$ (see Fig. 7):

(2) Consider an electron in the field of two nuclei; $\overrightarrow{\mathrm{F}}_{A}, \overrightarrow{\mathrm{F}}_{B}$ will be the forces acting from nucleus $A$ and $B$ on the electron. $\overrightarrow{\mathrm{F}}$ will be the resulting total force. If $\overrightarrow{\mathrm{F}}$ points directly at nucleus $A$, we say the electron belongs to $A$ and set $f=-1$. The analogue is true for $B$. If $\overrightarrow{\mathrm{F}}$, however, points to $\mathrm{CMN}$, we say the electron neither belongs to $A$ nor to $B$, and we set $\lambda+f$ $=0$. Generalizing this we set

$$
\frac{\lambda+f(\overrightarrow{\mathrm{r}}, \overrightarrow{\mathrm{R}})}{2}=\alpha
$$

where $\alpha$ is obtained from Fig. 7, as

$$
\alpha=\frac{\mu_{2}}{\mu_{1}}=\frac{M_{A}}{M_{N}} \frac{1-\left(Z_{A} / Z_{B}\right)\left(M_{B} / M_{A}\right)\left(r_{B} / r_{A}\right)^{3}}{1+\left(Z_{A} / Z_{B}\right)\left(r_{B} / r_{A}\right)^{3}} .
$$

One easily verifies that $\alpha$ obeys the correct boundary conditions:

(i) $r_{A} \rightarrow 0, R$ fixed $\Rightarrow \alpha \rightarrow-\frac{M_{B}}{M_{N}}=\frac{\lambda-1}{2}=\frac{\lambda+f_{A}}{2}$.

(ii) $r_{B} \rightarrow 0, R$ fixed $\Rightarrow \alpha \rightarrow \frac{M_{A}}{M_{N}}=\frac{\lambda+1}{2}=\frac{\lambda+f_{B}}{2}$.

(iii) $R \rightarrow 0, r_{A}, r_{B}$ fixed $\Rightarrow \alpha \rightarrow \frac{Z_{B} M_{A}-Z_{A} M_{B}}{M_{N}\left(Z_{A}+Z_{B}\right)}$.

[In Ref. 44 a slightly modified version of this

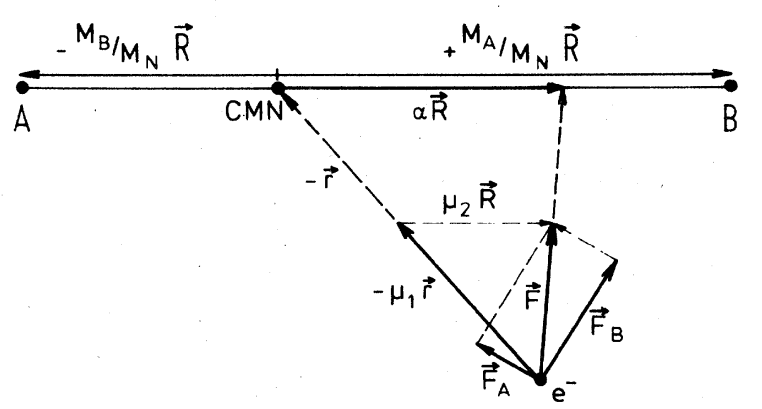

FIG. 7. Vector diagram of the forces acting on an electron by two nuclei with charges $Z_{A}, Z_{B}$. $f(\overrightarrow{\mathrm{r}}, \overrightarrow{\mathrm{R}})$ is presented which also in limit (iii) shows exactly the correct behavior.] Here, $\alpha$ only vanishes for systems with $Z_{A} / Z_{B}=M_{A} / M_{B}$. This is due to the fact that CMN is not identical with the center of charge. In our earlier consideration of the kinetic energy of the relative motion we naturally were led to the CMN as the origin of our coordinate system. Now arguing with the electric forces, the center of charge would be the natural origin. Since these differences, however, are small [in the limit (iii) $\alpha=4.5 \times 10^{-3}$ for $\mathrm{Pb}-\mathrm{Cm}$, $\alpha=3.5 \times 10^{-4}$ for $\mathrm{U}-\mathrm{Cm}$ collisions], we will not pursue this problem.

Now we have two forms for $f(\vec{r}, \overrightarrow{\mathrm{R}})$ that can be compared. To this end we optimized the parameter $g$ in (5.1) by numerically minimizing (5.2) for several states $\mid m>$. This was done in the U-Cm system for two nuclear distances $(R=35$ and 3000 fm); the $N=21$ lowest two-center states ${ }^{1} \Sigma$ to ${ }^{14} \Sigma$, ${ }^{1} \Pi$ to ${ }^{7} \Pi$ were taken into account. The resulting optimal parameter turned out to be by no means universal; it strongly depends on $R$ as well as on $|m\rangle$, thus violating the first criterion in Ref. 18 . Sometimes the minimum of (5.2) is so shallow that $g^{\text {opt }}$ even cannot be properly determined. In Table III we summarize the results for $g_{\mathrm{opt}}$. On the other hand, calculating with $g_{\text {opt }}$ the minimum of (5.2) always gave values very near (within $5 \%$ ) the result obtained by using the parameter-free ansatz (5.5). The deviations were in both directions such that neither of both Ansätze in its effect could be considered better.

This result lead us to the use of (5.4) and (5.5) in all practical calculations to be shown in the next section. This saved us from spending a lot of computer time for the optimization of the switching function. The good results obtained with the parameter-free ansatz (5.5) [compared to (5.1)] seem to indicate that the physical picture behind it (see Fig. 7) is essentially correct.

TABLE III. Results for $g_{\text {opt }}$.

\begin{tabular}{ccl}
\hline \hline$|m\rangle$ & $R(\mathrm{fm})$ & \multicolumn{1}{c}{$g_{\text {opt }}$} \\
\hline${ }^{1} \Sigma 1 s_{1 / 2} \sigma$ & 35 & $4.58 \times 10^{-2}$ \\
${ }^{3} \Sigma 2 s_{1 / 2} \sigma$ & 35 & $4.41 \times 10^{-2}$ \\
${ }^{2} \Sigma 2 p_{1 / 2} \sigma$ & 35 & $1.74 \times 10^{-2}$ \\
${ }^{8} \Sigma 3 d_{3 / 2} \sigma$ & 35 & $>14$ \\
${ }^{1} \Sigma 1 s_{1 / 2} \sigma$ & 3000 & 1.56 \\
${ }^{4} \Sigma 2 s_{1 / 2} \sigma$ & 3000 & $>.89$ \\
${ }^{5} \Sigma 3 d_{3 / 2} \sigma$ & 3000 & $>.80$ \\
\hline \hline
\end{tabular}




\section{THE INFLUENCE OF THE TRANSLATION CORRECTIONS ON THE $K$-HOLE PRODUCTION IN Pb-Cm COLLISIONS}

Using the parameter-free switching function discussed in the last section the translation matrix elements were calculated for the $\mathrm{Pb}-\mathrm{Cm}$ system. The two-center bound states for this system were calculated by $W$. Betz ${ }^{30}$; they will be published elsewhere. All matrix elements between the 11 lowest $\Sigma$ states and the 4 lowest $\Pi$ states were determined for nuclear distances between $R=16$ and $3100 \mathrm{fm}$. Thus in the united-atom limit the three lowest shells $(K, L, M)$ have been included completely, except for two $\Delta$ states. In Fig. 8 we show the correlation diagram for the $\mathrm{Pb}-\mathrm{Cm}$ system.

Motivated by an earlier investigation of matrix elements between molecular wavefunctions, ${ }^{5}$ in all
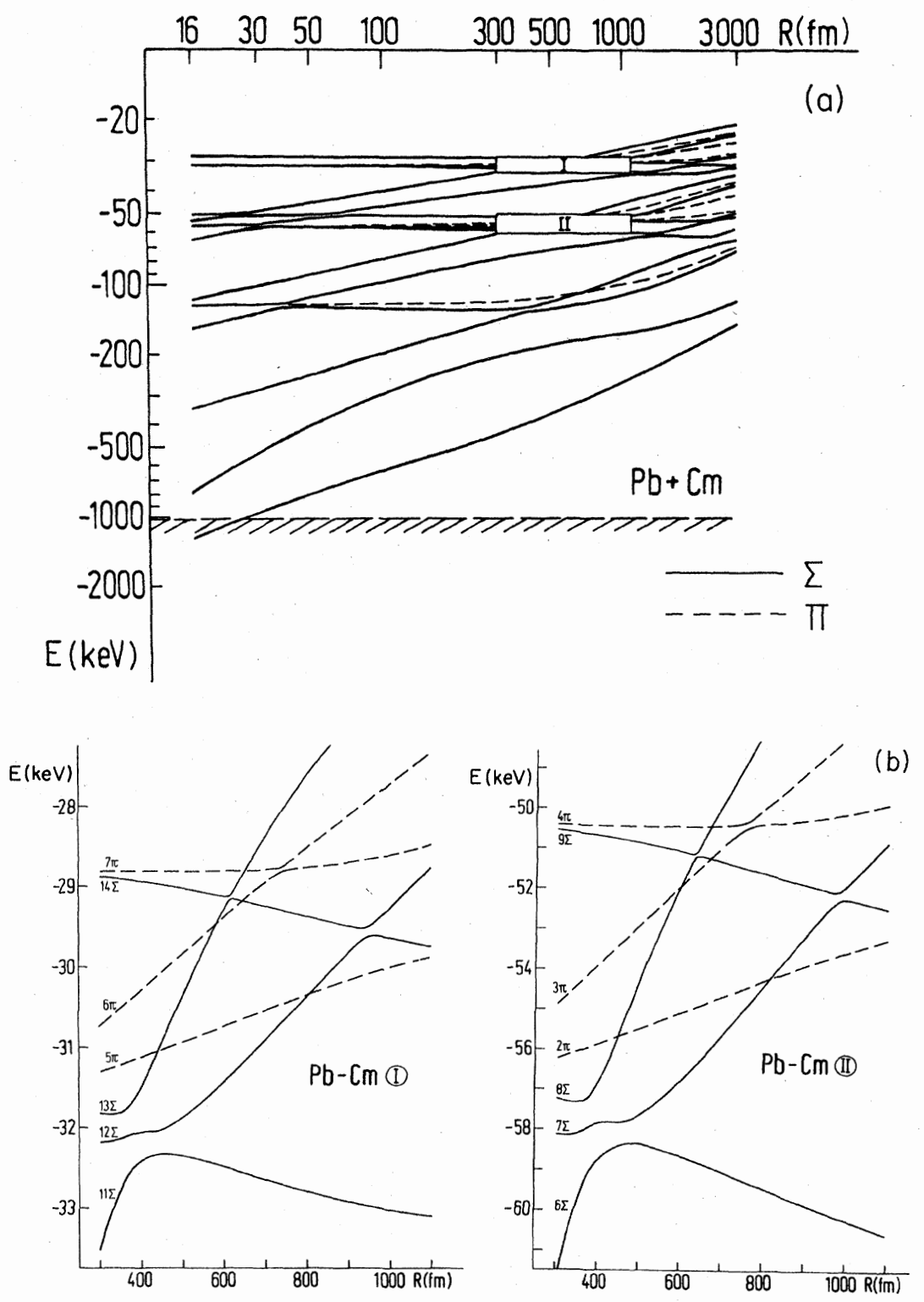

FIG. 8. (a) The correlation diagram for the $\mathrm{Pb}-\mathrm{Cm}$ system. $\Sigma(-)$ and $\Pi(---)$ states are numbered in continuous order from the bottom to the top of the diagram. (b) The crossing regions I and II of (a) are shown in detail. The figures are taken from Ref. 50. 


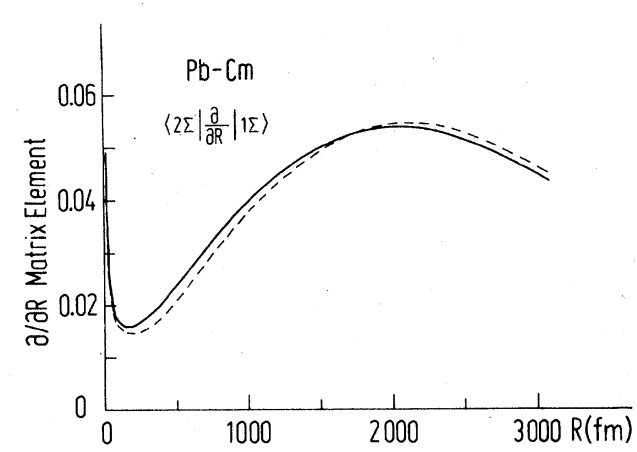

FIG. 9. The radial ${ }^{2} \Sigma-{ }^{1} \Sigma$ matrix element for the $\mathrm{Pb}-$ Cm system.

previous calculations by Kirsch, ${ }^{16}$ Reinhardt, ${ }^{14,33,34}$ and Soff ${ }^{15,45}$ the matrix elements were artificially cut off at $R \sim 1500-2000 \mathrm{fm}$. The resulting cross sections turned out to be rather insensitive to this cutoff procedure, if many channels were taken into account. However, in calculations with only a small number of channels the results showed strong dependence upon where the matrix elements were cut off. In the calculations presented below, the cutoff will be provided in a natural way by the translation corrections. Thus we can check the validity of the previously used cutoff methods. It turns out that all these conventional methods are unphysical and may in principle lead to arbitrary results (particularly in calculations where only a few channels are physically significant). The reason for that we will now investigate in some detail.

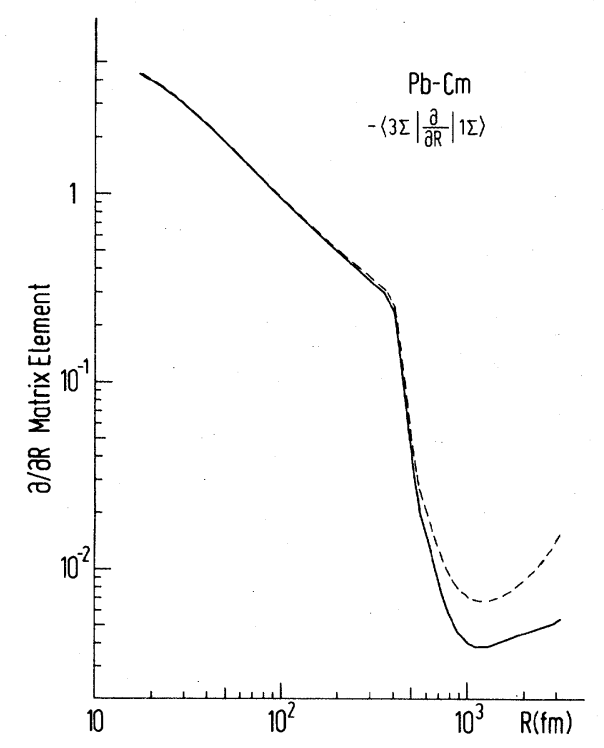

FIG. 10. The radial ${ }^{4} \Sigma-{ }^{1} \Sigma$ matrix element for the $\mathrm{Pb}-$ Cm system.

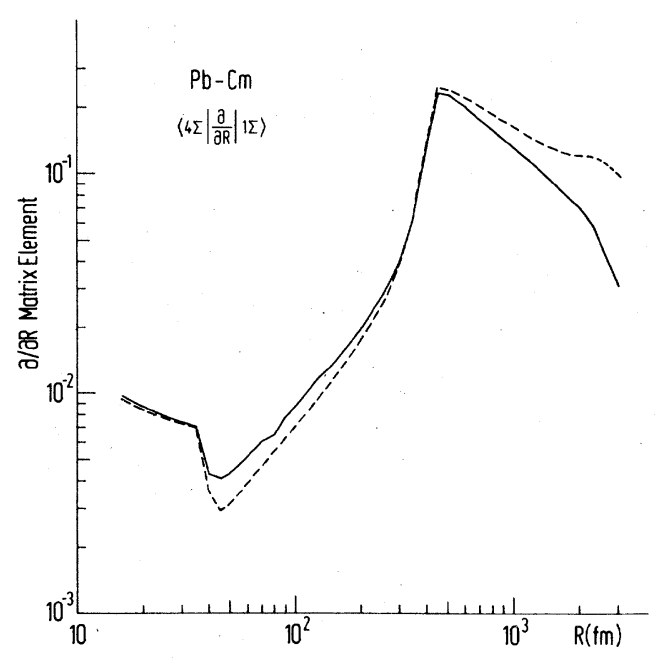

FIG. 11. The radial ${ }^{4} \Sigma-{ }^{1} \Sigma$ matrix element for the $\mathrm{Pb}-$ Cm system.

\section{A. The behavior of the translation matrix elements}

In Figs. 9 to 15 we show some of the corrected coupling matrix elements $(\underline{\overrightarrow{\mathrm{P}}}+\underline{\overrightarrow{\mathrm{A}}})_{m_{n}}$. In fact, for large nuclear distances nearly all corrected couplings are smaller than the uncorrected ones. Only for a small number of couplings the translation corrections at $R=3100 \mathrm{fm}$ do not yet show the expected behavior, namely, to decrease the coupling

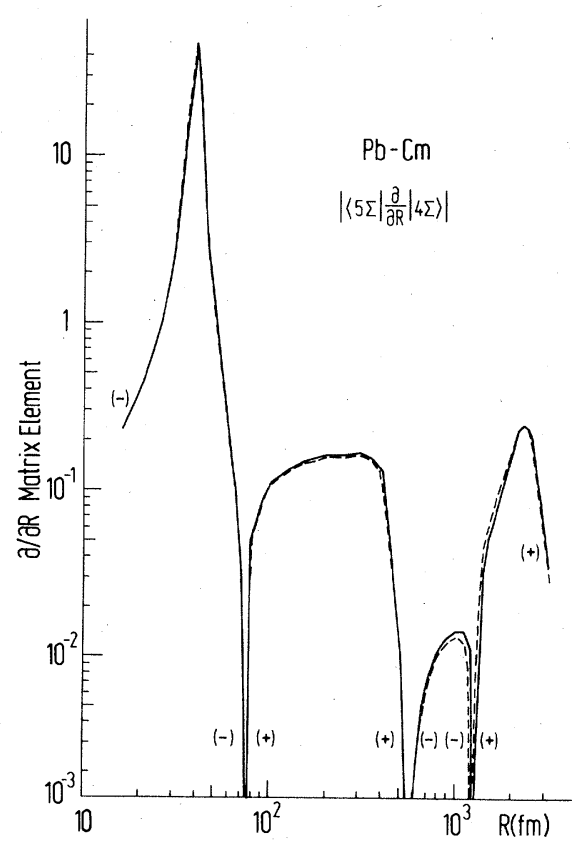

FIG. 12. The radial ${ }^{5} \Sigma-{ }^{4} \Sigma$ matrix element for the $\mathrm{Pb}-$ Cm system. 


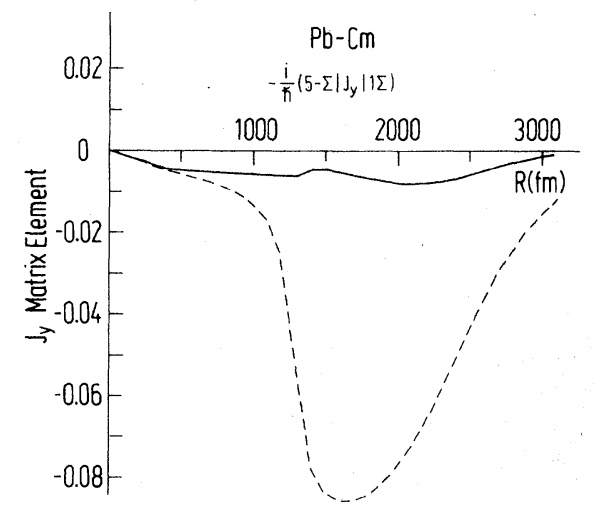

FIG. 13. The angular ${ }^{5}(-\Sigma)-{ }^{1} \Sigma$ matrix element for the $\mathrm{Pb}-\mathrm{Cm}$ system.

(see, e.g., Fig. 14 and 15). However, there are also only a few matrix elements where the corrections are really strong. The corrected matrix elements are typically a factor of 3 smaller than the uncorrected ones at $R=3100 \mathrm{fm}$. Also for states which intuitively could be considered as atomic at $R=3100 \mathrm{fm}$, there still occur large couplings, e.g., the radial ${ }^{2} \Sigma-{ }^{1} \Sigma$ coupling [asmyptotically $1 s(\mathrm{~Pb})-1 s(\mathrm{Cm})]$, which between 2000 and $3000 \mathrm{fm}$ shows the typical sharing behavior $\mathrm{r}^{42,46,47}$ (see Fig. 9) and is hardly influenced by the correction matrix elements; or the radial ${ }^{4} \Sigma-{ }^{1} \Sigma$ coupling [asymptotically $2 s(\mathrm{~Pb})-1 s(\mathrm{Cm})]$, which also is only reduced by a factor of 3 (see Fig. 11). Although the corrections in general have the correct sign their magnitude is smaller than expected. Obviously at $R=3100 \mathrm{fm}$ the $\mathrm{Pb}-\mathrm{Cm}$ system even for the inner shells does not behave asymptotically enough as to be referred to as separate atoms. The overlap of the wave functions is still too large for the translation matrix elements to be fully effective.

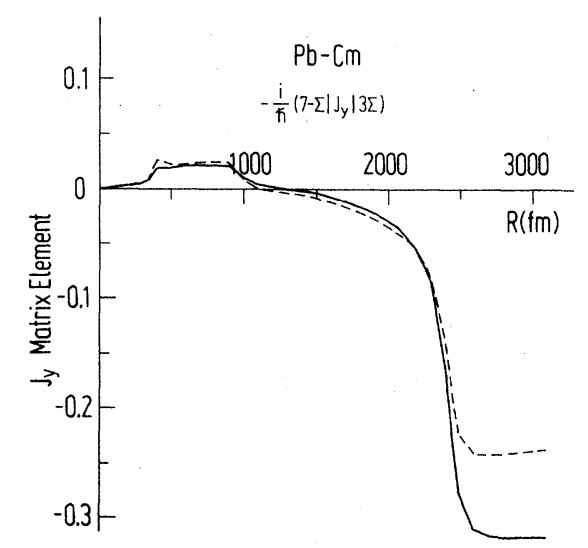

FIG. 14. The angular ${ }^{7}(-\Sigma)-{ }^{3} \Sigma$ matrix element for the $\mathrm{Pb}-\mathrm{Cm}$ system.

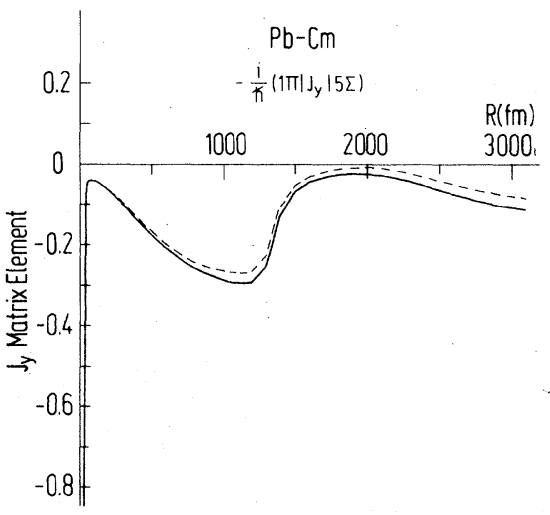

FIG. 15. The angular ${ }^{1} \Pi-{ }^{5} \Sigma$ matrix element for the $\mathrm{Pb}-\mathrm{Cm}$ system.

This conclusion is supported by the behavior of the sum of excitations in the ${ }^{1} \Sigma$ state (see Fig. 16). This sum,

$$
\frac{1}{N} \sum_{n=1}^{N} \frac{\left(\overrightarrow{\mathrm{P}}_{m n}+\overrightarrow{\mathrm{A}}_{m n}\right)^{2}}{\left(\overrightarrow{\mathrm{P}}_{m n}-\overrightarrow{\mathrm{A}}_{m n}\right)^{2}},
$$

is generally smaller than 1 for $R>400 \mathrm{fm}$. [Only at points, where level crossings occur and correspondingly some matrix elements show peaks which are not due to translational effects, (6.1) is larger than 1.] However, it decreases slowly (roughly like $R^{-0.8}$ ) and is still of the order of 0.2 at $R=3100 \mathrm{fm}$.

\section{B. The $K$-hole amplitude in $\mathrm{Pb}-\mathrm{Cm}$ collisions}

Recent measurements of the $K$-hole probability in $\mathrm{Pb}-\mathrm{Cm}$ collisions ${ }^{48}$ show discrepancies to the calculations in monopole approximation by Soff

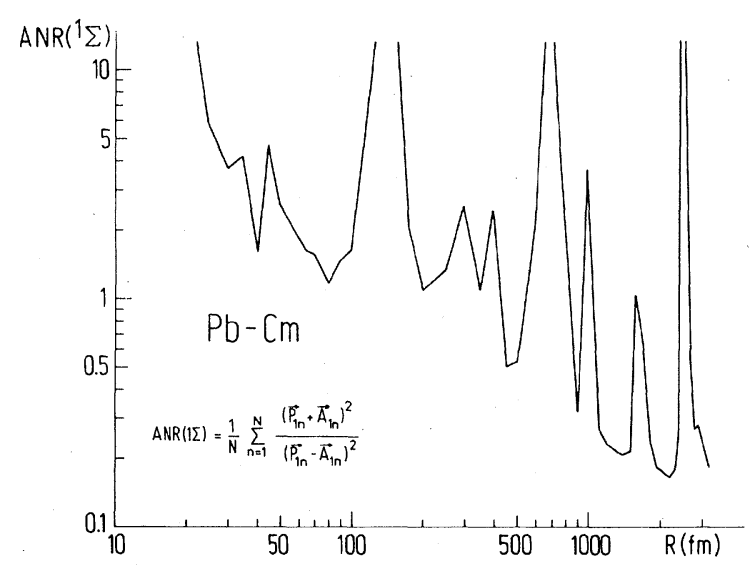

FIG. 16. The sum of excitations in the ${ }^{1} \Sigma$ state of the $\mathrm{Pb}-\mathrm{Cm}$ system. The strong maxima are due to level crossings, where some matrix elements show strong structure and then dominate the excitation. 
et $a l . .^{49}$ at small impact parameters. It was suspected that these discrepancies can be traced to neglect of the rotational coupling in the monopole approximation and that calculations using the full two-center wave functions are necessary. ${ }^{50}$ Preliminiary calculations using only the three inner shells fail to reproduce the experimental data. ${ }^{50}$ Full calculations, on the other hand, including coupling to the continuum, are still missing. However, to see the effect of translation corrections, the existing preliminary calculations may be compared to corr esponding computations where the corrected matrix elements $(\underline{\vec{A}}+\underline{\overrightarrow{\mathbf{P}}})_{m n}$ are used. To this end we made a test calculation taking into account only three states: ${ }^{1} \Sigma,{ }^{3} \Sigma$, and ${ }^{4} \Sigma$. Only radial coupling was considered. We did the calculation for two energies $\left(E_{\mathrm{lab}}=3.6\right.$ and $5.9 \mathrm{MeV}$ / amu and for three impact parameters $(b=20,50$, $100 \mathrm{fm}$ ) and compared the result with corresponding calculations using (a) the monopole approximation, and (b) two-center matrix elements which were exponentially cut off at $1500 \mathrm{fm} .^{50}$ The different $K$-hole probabilities are shown in Fig. 17.

As the figure shows the three different models lead to vastly different results, in particular for large impact parameters. On the other hand, the results of model (b) vary in the same range if the point where the matrix elements are cut off is changed. ${ }^{50}$ The reason is easily realized by looking at the occupation probability of the ${ }^{1} \Sigma$ state

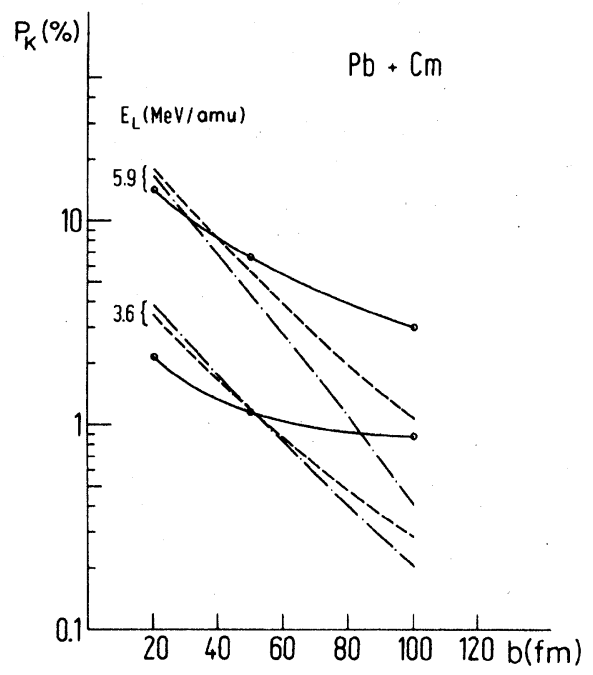

FIG. 17. The $K$-hole probability for a test calculation with a three-state system. The ${ }^{1} \Sigma,{ }^{2} \Sigma$, and ${ }^{4} \Sigma$ states were considered; in the ingoing channel only ${ }^{1} \Sigma$ was occupied. The curves show results of the monopole approximation (---), the two center calculation with exponentially cut-off matrix elements (-.--) and with matrix elements corrected for translation effects (-). $\left|a_{1 \Sigma}(R)\right|^{2}$ as a function of $R$ (see Fig. 18). At $R \cong 3000 \mathrm{fm}$ this probability still oscillates with an amplitude which is in agreement with the observed variations of $P(b)$ as the cutoff point is varied. Thus the reason for $P(b)$ being ill defined in calculations which extend only to $R=3100$ $\mathrm{fm}$ is that the coupling matrix elements are still too large at this internuclear distance, although translation corrections have been taken into account.

There are several consequences to be drawn from this result.

(1) The often used way of cutting off the matrix elements at $R \sim 1500-3000 \mathrm{fm}$ is unphysical. In special cases (i. e., if only very few transitions contribute to the cross section), the resulting calculated cross sections may be unreliable. Hence the exact knowledge of the matrix elements for large $R$ is necessary (in particular for collisions with large impact parameter), and the translation corrections play an important role.

(2) Cutting off the dynamical coupling matrix elements $\overrightarrow{\mathrm{P}}_{m n}$ in a natural way by taking into account the translation effect via $\overrightarrow{\mathrm{A}}_{m n}$ requires the knowledge of the two-center electron states up to much larger nuclear distances $R$ (presumably $>10000 \mathrm{fm}$ ). The asymptotic excitation amplitudes seem to be only well defined if the two ions are several $(\approx 10)$ times the $K$-shell radius apart.

(3) Even the knowledge of the way in which $(\underline{\overrightarrow{\mathrm{P}}}+\underline{\overrightarrow{\mathrm{A}}})_{m n}$ for $R \rightarrow \infty$ approaches zero does not help us, since we do not know how to match this asymptotic behavior to the calculated matrix elements at $R<3000 \mathrm{fm}$. As demonstrated in Appendix $\mathrm{B}$, $(\underline{\overrightarrow{\mathrm{P}}}+\underline{\overrightarrow{\mathrm{A}}})_{m_{n}}$ falls asymptotically like $\beta R^{-3}$. This behavior of the matrix elements, however, cannot be observed at distances $R \lesssim 3000 \mathrm{fm}$ so that the proportionality constant $\beta$ cannot be determined by just looking at the matrix elements. They are not yet asymptotic enough in the considered range of $R$. It is not difficult to show ${ }^{24}$ that just fitting

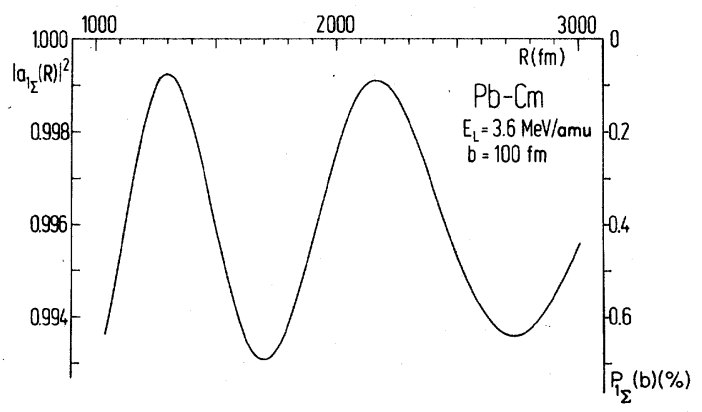

FIG. 18. The occupation amplitude of the ${ }^{1} \Sigma$ state as a function of the nuclear distance in the outgoing channel for the same test calculation as in Fig. 17. 
the asymptotic behavior $\beta R^{-3}$ to the matrix elements at some point $R_{0} \varsigma 3100 \mathrm{fm}$ yields similarly unreliable results as the conventional cutoff methods: as $R_{0}$ is varied, $P(b)$ varies in the same order of magnitude as before.

(4) Inclusion of couplings to higher states and to the continuum may make the cross sections less sensitive to the cutoff procedure (as was the case in the monopole calculations ${ }^{15,45}$ ). This may, however, mean that they are also less sensitive to translation effects. This question has still to be checked by more extensive calculations.

At this point a remark is in order concerning the many calculations of electronic excitations which were done using the monopole approximation for the two-center potential. ${ }^{14,15,34,49}$ There, also, the coupling matrix elements usually have been cut off at internuclear distances of $R \sim 1500$ $2000 \mathrm{fm}$. This cutoff is motivated by the fact that the large- $R$ behavior of the matrix elements in monopole approximation is wrong, anyway, due to neglect of two-center effects, and that the main contribution to electronic excitations come from small nuclear distances. Hence the error made by not correctly taking into account translation effects in that case is much less serious than the error intrinsic to the monopole approximation itself. Why the monopole approximation is working so well, and in many cases yields results in extremely good agreement with experiment, ${ }^{49}$ is not yet understood. In this paper, however, we did not show that the monopole approximation is wrong, but that doing two-center calculations without translation corrections is wrong. The validity of the monopole approximation is another problem.

\section{CONCLUSIONS}

In this paper we reviewed the theory of electronic excitations in heavy-ion collisions, starting from a basic quantum-mechanical treatment. Employing a number of approximations, each of which has been thoroughly investigated as to its validity, we finally derived a set of coupled differential equations for the occupation amplitudes of the molecular electronic states. These coupled-channel equations consistently contain the lowest-order corrections from electron-translation effects, and thus yield well-defined asymptotic occupation probabilities. No spurious long-range dynamical couplings occur.

The difficulties with the treatment of continuum electrons by two-center continuum wave functions were avoided by constructing another continuum basis. We use, as continuum wave functions, wave packets constructed from quasiatomic Coulomb wave functions, which in the limit $R \rightarrow 0$ be- come the well-known two-center continuum wave functions in monopole approximation, and for $R \rightarrow \infty$ approach atomic Coulomb waves belonging to one of the two nuclei. The wave packets used were shown to fall off fast enough at infinity in order to show no asymptotic couplings with the two-center potential. For high energetic continuum states, also the matrix elements from the nonorthogonality with the bound states vanish for $R \rightarrow \infty$. The very-low-energy part of the continuum spectra, however, cannot be well described in our basis. Nevertheless, we consider this formulation to be an essential progress toward a useful theory of electronic excitations into the continuum, since it was shown to be powerful enough to yield (to our knowledge for the first time) also the angular distribution of the continuum spectra.

As a first application of our theory we investigated the $K$-hole production in $\mathrm{Pb}-\mathrm{Cm}$ collisions. In our preliminary calculations we neglected excitations to the continuum and only took into account the inner shells, since in this first step we were primarily interested in how far the dynamical couplings between these states were modified by electron-translation corrections. For the computation of the translational matrix elements we used a parameter-free switching function derived from a consideration of the electric forces by the two nuclei acting on the electron. It was shown that for large nuclear distances the dynamical couplings were reduced by the translation corrections, but not as strongly as we expected. A full cancellation of the asymptotic dynamical couplings only occurs for very large nuclear distances (we estimate $R>10000 \mathrm{fm}$ for the $\mathrm{Pb}-\mathrm{Cm}$ system).

Thus our test calculations showed that the conventional method of artificially cutting off the dynamical coupling matrix elements at $R \sim 1500-3000$ $\mathrm{fm}$ has to be used with great care. We showed by numerical solution of the coupled-channel equations that the exact behavior of the corrected matrix elements at large nuclear distances may strongly influence the $K$-hole production probabilities, in particular for collisions with large impact parameter. This shows the importance of electrontranslation effects. Whether a full calculation, also taking into account higher bound and continuum states, will be similarly sensitive to the large $R$ behavior of the matrix elements remains to be checked numerically.

\section{ACKNOWLEDGMENTS}

We wish to thank Wilfried Betz very much for providing us with the two-center wave functions and matrix elements for the $\mathrm{Pb}-\mathrm{Cm}$ system prior to publication. We thank Dr. J. Kirsch for leaving us his computer program for the solution of the 
coupled-channel equations. We gratefully acknowledge discussions with Dr. J. Kirsch and Dr. G. Soff. Two of us (U. H. and W. G.) thank the WNSL at Yale University, where this paper was comple- ted, for their kind hospitality. This work was supported by Deutsche Forschungsgesellschaft (DFG) and Gesellschaft fiir Schwerionenforschung (GSI); it is part of the Ph.D. thesis of one of us (U. H.).

\section{APPENDIX A: THE TRANSFORMED HAMILTONIAN $\boldsymbol{H}^{\prime}(\overrightarrow{\mathbf{r}}, \overrightarrow{\mathbf{R}})$}

We first note that $H^{\prime}=\left(\hat{T}^{+} \hat{T}\right)^{1 / 2} \hat{T}^{-1} H \hat{T}\left(\hat{T}^{+} \hat{T}\right)^{-1 / 2}$ must be Hermitian, as $\left(\hat{T}^{+} \hat{T}\right)^{1 / 2} \hat{T}^{-1}$ is a unitary operator. Using (2.19) and (2.20) and (2.25) and (2.26) we calculate $\hat{T}^{+}(\overrightarrow{\mathrm{X}}) \hat{T}(\overrightarrow{\mathrm{X}})$ :

$$
\begin{aligned}
\hat{T}^{+}(\overrightarrow{\mathrm{X}}) \hat{T}(\overrightarrow{\mathrm{X}}) \cong(1-(i / \hbar) \overrightarrow{\mathrm{P}} \cdot \overrightarrow{\mathrm{X}})(1+(i / \hbar) \overrightarrow{\mathrm{X}} \cdot \overrightarrow{\mathrm{P}}) \cong 1-(i / \hbar)[\overrightarrow{\mathrm{P}}, \overrightarrow{\mathrm{X}}] \\
\cong 1+\frac{3}{8} \frac{m}{\mu} \sum_{i=1}^{z^{\prime}}\left[f\left(\overrightarrow{\mathrm{r}}_{i}, \overrightarrow{\mathrm{R}}\right)+\lambda\right]^{2} \equiv 1+\frac{3}{8} \frac{m}{\mu} g(\overrightarrow{\mathrm{r}}, \overrightarrow{\mathrm{R}})
\end{aligned}
$$

where we have defined $g(\overrightarrow{\mathbf{r}}, \overrightarrow{\mathbf{R}})=\Sigma_{i}\left[f\left(\overrightarrow{\mathbf{r}}_{i}, \overrightarrow{\mathbf{R}}\right)+\lambda\right]^{2}$. Thus in $O(m / \mu),\left(\hat{T}^{+} \hat{T}\right)^{-1 / 2}$ commutes with the rest up to terms $\sim(m / \mu) \operatorname{grad} f$, which we neglect, and we have

$$
H^{\prime} \cong \hat{T}^{-1} H \hat{T}=H+\hat{T}^{-1}[H, \hat{T}] .
$$

Remembering that

$$
\hat{T}^{-1}(\overrightarrow{\mathrm{X}})=\hat{T}(-\overrightarrow{\mathrm{X}})=1-(i / \hbar) \overrightarrow{\mathrm{X}} \cdot \overrightarrow{\mathbf{P}}+\cdots,
$$

we now may calculate $H^{\prime}$ in $O(\mathrm{~m} / \mu)$ :

(i) The operator $P^{2} / 2 \mu$ is transformed into

$$
\begin{aligned}
\frac{1}{2 \mu}[1-(i / \hbar) \overrightarrow{\mathrm{X}} \cdot \overrightarrow{\mathrm{P}}] P^{2} & {[1+(i / \hbar) \overrightarrow{\mathrm{X}} \cdot \overrightarrow{\mathrm{P}}] } \\
\cong & \frac{P^{2}}{2 \mu}+\frac{1}{2 \mu}(i / \hbar)\left[P^{2}, \overrightarrow{\mathrm{X}}\right] \cdot \overrightarrow{\mathrm{P}} .
\end{aligned}
$$

Neglecting terms $\sim(m / \mu) \vec{\nabla} f$ the commutator in (A4) vanishes, and we have

$$
\frac{P^{\prime 2}}{2 \mu} \cong \frac{P^{2}}{2 \mu} .
$$

(ii) The kinetic part of $H_{\mathrm{mol}}^{\mathrm{el}}(\overrightarrow{\mathbf{r}}, \overrightarrow{\mathbf{R}})$ is treated as follows:

$$
\begin{aligned}
& {[1-(i / \hbar) \overrightarrow{\mathrm{X}} \cdot \overrightarrow{\mathbf{P}}]\left(H_{\mathrm{mol}}^{\mathrm{el}}\right)_{\mathrm{kin}}[1+(i / \hbar) \overrightarrow{\mathrm{X}} \cdot \overrightarrow{\mathbf{P}}] } \\
& \cong\left(H_{\mathrm{mol}}^{\mathrm{el}}\right)_{\mathrm{kin}}+(i / \hbar)\left[\left(H_{\mathrm{mol}}^{\mathrm{el}}\right)_{\mathrm{kin}}, \overrightarrow{\mathrm{X}}\right] \cdot \overrightarrow{\mathbf{P}} \\
& \equiv\left(H_{\mathrm{mol}}^{\mathrm{el}}\right)_{\mathrm{kin}}+\frac{1}{\mu} \overrightarrow{\mathrm{A}} \cdot \overrightarrow{\mathbf{P}},
\end{aligned}
$$

where

$$
\overrightarrow{\mathrm{A}}=\mu(i / \hbar)\left[\left(H_{\mathrm{mol}}^{\mathrm{el}}\right)_{\mathrm{kin}}, \overrightarrow{\mathrm{X}}\right]=\mu(i / \hbar)\left[H_{\mathrm{mol}}^{\mathrm{el}}, \overrightarrow{\mathrm{X}}\right] .
$$

The term $(1 / \mu) \vec{A} \cdot \vec{P}$ is a small, but asymptotically important, correction to the kinetic energy $P^{2} / 2 \mu$ of the nuclear relative motion. It is Hermitian up to terms which are a factor $\operatorname{grad}_{\vec{R}} f$ smaller, and therefore may be neglected. (The result would have been explicitly Hermitian had we not neglected similar terms when commuting $\left(\hat{T}^{+} \hat{T}\right)^{-1 / 2}$ with $\left.\hat{T}^{-1} H \hat{T}\right)$. Hermiticity may therefore be made manifest by writing in $O(\mathrm{~m} / \mu)$

$$
\left(H_{\mathrm{mol}}^{\mathrm{el}}\right)_{\mathrm{kin}}^{\prime}=\left(H_{\mathrm{mol}}^{\mathrm{el}}\right)_{\mathrm{kin}}+\frac{1}{2 \mu}\{\overrightarrow{\mathrm{A}}, \overrightarrow{\mathrm{P}}\} \text {. }
$$

(iii) Finally, we consider the potential. For any function $F(\overrightarrow{\mathrm{R}})$ we have:

$$
\begin{aligned}
\hat{T}^{-1}(\overrightarrow{\mathbf{X}}) V(\overrightarrow{\mathbf{R}}) \hat{T}(\overrightarrow{\mathbf{X}}) F(\overrightarrow{\mathbf{R}}) & =\hat{T}(-\overrightarrow{\mathbf{X}}) V(\overrightarrow{\mathbf{R}}) F(\overrightarrow{\mathbf{R}}+\overrightarrow{\mathbf{X}}) \\
& =V(\overrightarrow{\mathbf{R}}-\overrightarrow{\mathbf{X}}) F(\overrightarrow{\mathbf{R}})
\end{aligned}
$$

hence

$$
\hat{T}^{-1}(\overrightarrow{\mathrm{X}}) V(\overrightarrow{\mathrm{R}}) \hat{T}(\overrightarrow{\mathrm{X}})=V(\overrightarrow{\mathrm{R}}-\overrightarrow{\mathrm{X}}) .
$$

The transformed Hamiltonian thus reads:

$$
\begin{aligned}
H^{\prime}(\overrightarrow{\mathrm{r}}, \overrightarrow{\mathrm{R}})= & H(\overrightarrow{\mathrm{r}}, \overrightarrow{\mathrm{R}})+\frac{1}{2 \mu}\{\overrightarrow{\mathrm{A}}, \overrightarrow{\mathrm{P}}\}+\Delta \\
& =\frac{P^{2}}{2 \mu}+V^{A B}(R)+H_{\mathrm{mol}}^{\mathrm{el}}(\overrightarrow{\mathrm{r}}, \overrightarrow{\mathrm{R}})+\frac{1}{2 \mu}\{\overrightarrow{\mathrm{A}}, \overrightarrow{\mathrm{P}}\}+\Delta,
\end{aligned}
$$

where $\Delta$ is given by (2.33). $H^{\prime}$ is Hermitian.

\section{APPENDIX B: THE CORRECTED MATRIX ELEMNTS FOR $R \rightarrow \infty$}

For simplicity we assume a one-electron system. The molecular basis is constructed from solutions of

$$
\left(\vec{\alpha} \cdot \overrightarrow{\mathrm{p}}+\beta m_{0}+V^{e A}+V^{e B}\right) \varphi_{m}(\overrightarrow{\mathrm{r}}, \mathrm{R})=\epsilon_{m}(R) \varphi_{m}(\overrightarrow{\mathrm{r}}, R) .
$$

An atomic basis around nucleus $A$ is defined by

$$
\left[\vec{\alpha} \cdot \overrightarrow{\mathrm{p}}_{A}+\beta m_{0}+V^{e A}\left(r_{A}\right)\right] \varphi_{n}^{A}\left(\overrightarrow{\mathrm{r}}_{A}\right)=\epsilon_{n}^{A} \varphi_{n}^{A}\left(\overrightarrow{\mathrm{r}}_{A}\right) .
$$

There is an analogous construction of a basis around nucleus $B$. (B.1) can be rewritten as

$$
\begin{aligned}
\left\{\left(\vec{\alpha} \cdot \overrightarrow{\mathrm{p}}_{A}+\beta m_{0}+V^{e A}\right)+\left[V^{e B}+\vec{\alpha} \cdot\left(\overrightarrow{\mathrm{p}}-\overrightarrow{\mathrm{p}}_{A}\right)\right]\right\} \varphi_{m}(\overrightarrow{\mathrm{r}}, R) \\
=\epsilon_{m}(R) \varphi_{m}(\overrightarrow{\mathrm{r}}, R) .
\end{aligned}
$$

This allows for large $R$, where $V^{* B}$ is small at the location of nucleus $A$, a perturbation expansion [according to Sec. II, $\vec{\alpha} \bullet\left(\vec{p}-\vec{p}_{A}\right)$ is smaller than $\vec{\alpha} \cdot \vec{p}$ by a factor $m / \mu$ anyway]: 
$\varphi_{m}(\overrightarrow{\mathrm{r}}, R)=\varphi_{m}^{A}\left(\overrightarrow{\mathrm{r}}_{A}\right)+\sum_{n \neq m} \frac{\left\langle\varphi_{n}^{A}\left|V^{e B}+\alpha \cdot\left(\overrightarrow{\mathrm{p}}-\overrightarrow{\mathrm{p}}_{A}\right)\right| \varphi_{m}^{A}\right)}{\epsilon_{m}^{A}-\epsilon_{n}^{A}} \varphi_{n}^{A}\left(\overrightarrow{\mathrm{r}}_{A}\right)+\cdots$

Inserting this expansion into the corrected matrix element and using

$$
(\overrightarrow{\mathrm{P}}+\overrightarrow{\mathrm{A}}) \varphi_{m}^{A}=0+O\left(\frac{m}{\mu} \operatorname{grad} f\right),
$$

we obtain

$$
\begin{aligned}
\left\langle\varphi_{n}(\overrightarrow{\mathrm{r}}, R)|\overrightarrow{\mathrm{P}}+\overrightarrow{\mathrm{A}}| \varphi_{m}(\overrightarrow{\mathrm{r}}, R)\right\rangle & \cong \sum_{l \neq m}\left\langle\varphi_{n}(\overrightarrow{\mathrm{r}}, R)\left|(\overrightarrow{\mathrm{P}}+\overrightarrow{\mathrm{A}}) \frac{\left\langle\varphi_{l}^{A}\left|V^{e B}+\vec{\alpha} \cdot\left(\overrightarrow{\mathrm{p}}-\overrightarrow{\mathrm{p}}_{A}\right)\right| \varphi_{m}^{A}\right\rangle}{\epsilon_{m}^{A}-\epsilon_{l}^{A}}\right| \varphi_{l}^{A}\left(\overrightarrow{\mathrm{r}}_{A}\right)\right\rangle \\
& \cong \sum_{l \neq m}\left\langle\varphi_{n}(\overrightarrow{\mathrm{r}}, R) \mid \varphi_{l}^{A}\left(\overrightarrow{\mathrm{r}}_{A}\right)\right\rangle(\overrightarrow{\mathrm{P}}+\overrightarrow{\mathrm{A}}) \frac{\left\langle\varphi^{A}\left|V^{\boldsymbol{B} B}+\vec{\alpha} \cdot\left(\overrightarrow{\mathrm{p}}-\overrightarrow{\mathrm{p}}_{A}\right)\right| \varphi_{m}^{A}\right\rangle}{\epsilon_{m}^{A}-\epsilon_{l}^{A}}
\end{aligned}
$$

In (B7) the differential operator $\vec{P}+\vec{A}$ only acts on the matrix element following it. Using (B4) again finally yields

$$
\langle n|\overrightarrow{\mathrm{P}}+\overrightarrow{\mathrm{A}}| m\rangle \cong \sum_{l \neq m}\langle n \mid l, A\rangle \frac{1}{\epsilon_{m}^{A}-\epsilon_{l}^{A}}\left\langle l, A\left|(\overrightarrow{\mathrm{P}}+\overrightarrow{\mathrm{A}}) V^{e B}+(\overrightarrow{\mathrm{P}}+\overrightarrow{\mathrm{A}})\left[\vec{\alpha} \cdot\left(\overrightarrow{\mathrm{p}}-\overrightarrow{\mathrm{p}}_{A}\right)\right]\right| m, A\right\rangle .
$$

In this formula we neglect the matrix element of $(\vec{P}+\vec{A})[\vec{\alpha} \cdot(\vec{p}-\vec{p})]$, which is by a factor $(m / \mu)^{2}$ smaller than the expectation value of $\vec{\alpha} \cdot \vec{p}$. Now we have two possibilities for the wave function $\varphi_{n}$ :

(1) $\varphi_{n}(\overrightarrow{\mathrm{r}}, R) \underset{R \rightarrow \infty}{\longrightarrow} \varphi_{n}^{A}\left(\overrightarrow{\mathrm{r}}_{A}\right)$. In this case first-order perturbation theory gives

$$
\langle n|\overrightarrow{\mathrm{P}}+\overrightarrow{\mathrm{A}}| m\rangle \cong \frac{\left\langle n, A\left|(\overrightarrow{\mathrm{P}}+\overrightarrow{\mathrm{A}}) V^{e B}\right| m, A\right\rangle}{\epsilon_{m}^{A}-\epsilon_{n}^{A}} .
$$

(2) $\varphi_{n}(\overrightarrow{\mathrm{r}}, R) \underset{R \rightarrow \infty}{\longrightarrow} \varphi_{n}^{B}\left(\overrightarrow{\mathrm{r}}_{B}\right)$. Then we get

$$
\langle n|\overrightarrow{\mathrm{P}}+\overrightarrow{\mathrm{A}}| m\rangle \cong \sum_{l \neq m}\langle n, B \mid l, A\rangle \frac{\langle l, A|(\overrightarrow{\mathrm{P}}+\overrightarrow{\mathrm{A}})| m, A\rangle}{\epsilon_{m}^{A}-\epsilon_{l}^{A}} .
$$

In both cases matrix elements of the form (B9) play a role. We will investigate them in a moment. In case (2) also the overlap of the two atomic states $\langle n, B \mid l, A\rangle$ is important. It will vanish quite suddenly at some definite $R$ value, since there the exponentially decreasing tails of the two wave functions will cease to over lap. The magnitude of this separation distance $R$ depends on the quantum number $l$ of the intermediate state. If in the sum of (B10) also excitations into states with high $l$ will take place, then the separation distance $R$ may be quite large.

We now study formula (B9). We substitute $\overrightarrow{\mathrm{P}}+\overrightarrow{\mathrm{A}}$ by $\overrightarrow{\mathrm{P}}_{A B}$, which is correct up to terms $\sim(\mathrm{m} / \mu) \operatorname{grad} f$. Because of

$$
V^{e B}\left(r_{B}\right)=-\frac{Z_{B} e^{2}}{r_{B}}=-\frac{Z_{B} e^{2}}{\left|\overrightarrow{\mathrm{r}}_{A}-\overrightarrow{\mathrm{R}}\right|}=-\frac{Z_{B} e^{2}}{\left|\overrightarrow{\mathrm{r}}_{A}-\overrightarrow{\mathrm{R}}_{A B}\right|}+O\left(\frac{m}{\mu}\right),
$$

we get, as soon as the two atoms have separated $\left(R_{A B}>r_{A}\right)$,

$$
V^{e B}\left(r_{B}\right) \cong-Z_{B} e^{2} \sum_{i} \frac{(-)^{l} r_{A}^{l}}{R_{A B}^{l+1}} P_{l}(\cos \vartheta)
$$

Applying $\overrightarrow{\mathrm{P}}_{A B}$, the dominant term for large $R_{A B}$ is the dipole term, and we find

$$
\text { (B9) }-\frac{2 Z_{B} e^{2}}{\epsilon_{m}^{A}-\epsilon_{n}^{A}} \frac{\overrightarrow{\mathrm{R}}_{A B}}{R_{A B}^{4}}\left\langle n, A\left|r_{A} \cos \vartheta\right| m, A\right\rangle,
$$

In the same way we find for (B10):

$$
(\mathrm{B} 10) \rightarrow \frac{2 Z_{B} e^{2} \overrightarrow{\mathrm{R}}_{A B}}{R_{A B}^{4}} \sum_{l \neq m}\langle n, B \mid l, A\rangle \frac{\left\langle l, A\left|r_{A} \cos \vartheta\right| m, A\right\rangle}{\epsilon_{m}^{A}-\epsilon_{l}^{A}}
$$

This shows that for large nuclear distances the corrected matrix elements decrease at least like $1 / R^{3}$. The proportionality constant is given by the atomic dipole matrix elements within the separated atoms. How well the matrix elements behave like $1 / R^{3}$ is determined by the degree of separation of the two atoms. 
*Permanent address: Institut für Theoretische Physik, J.-W.-Goethe-Universität, Frankfurt am Main, West Germany.

${ }^{1} \mathrm{~J}$. Reinhardt and W. Greiner, Rep. Prog. Phys. $\underline{40}, 219$ (1977), and references therein.

${ }^{2}$ J. Theis, J. Reinhardt, and B. Müller, J. Phys. B 12 , L479 (1979).

${ }^{3}$ U. Fano and W. Lichten, Phys. Rev. Lett. 14, 627 (1965).

${ }^{4}$ B. Müller and W. Greiner, Z. Naturforsch. 31a, 1 (1976).

${ }^{5}$ W. Betz, G. Soff, B. Müller, and W. Greiner, Phys. Rev. Lett. 37, 1046 (1974); G. Soff, J. Reinhardt, and W. Betz, Phys. Scr. 17, 417 (1978).

${ }^{6}$ W. E. Meyerhof, T. K. Saylor, S. M. Lazarus, W. A. Little, B. B. Triplett, and L. F. Chase, Phys. Rev. Lett. 30, 1279 (1973).

'J. S. Greenberg, C. K. Davis, and P. Vincent, Phys. Rev. Lett. 33, 473 (1974).

${ }^{8}$ G. Kraft, P. H. Mokler, and H. J. Stein, Phys. Rev. Lett. 33, 476 (1974).

${ }^{9}$ B. Müller and W. Greiner, Phys. Rev. Lett. 33, 469 (1974); B. Müller, R. K. Smith, and W. Greiner, Phys. Lett. 49B, 219 (1974).

${ }^{10} \mathrm{~J}$. Kirsch, W. Betz, J. Reinhardt, G. Soff, B. Müller, and W. Greiner, Phys. Lett. 72B, 298 (1978).

${ }^{11}$ W. Wölfli, Ch. Stoller, G. Bonani, M. Suter, and M. Stöckli, Nuovo Cimento Lett. 14, 577 (1975); Ch. Stoller, W. Wölfli, G. Bonani, M. Stöckli, and M. Suter, J. Phys. B 10, L347 (1977); Ch. Stoller, W. Wölfli, G. Bonani, E. Morenzoni, and M. Stöckli, Z. Phys. A 287, 33 (1978).

${ }^{12}$ W. Pieper and W. Greiner, Z. Phys. 218, 327 (1969); V. S. Popov, Zh. Eksp. Teor. Fiz. 59, 965 (1970) [Sov. Phys-JETP 32, 526 (1971)].

${ }^{13}$ B. Müller, J. Rafelski, and W. Greiner, Z. Phys. 257, 62 (1972); 257, 183 (1972).

${ }^{14} \mathrm{~J}$. Reinhardt, Ph.D. thesis, Institut für theoretische Physik der Universität Frankfurt am Main, 1980 unpublished; see also Ref. 33(b).

${ }^{15}$ G. Soff, W. Greiner, W. Betz, and B. Müller, Phys. Rev. A 20, 169 (1979).

${ }^{16} \mathrm{~J}$. Kirsch, W. Betz, J. Reinhardt, B. Müller, W. Greiner, and G. Soff, Z. Phys. A 292, 227 (1979).

${ }^{17}$ D. R. Bates and R. McCarroll, Proc. R. Soc. London A245, 175 (1958).

${ }^{18}$ S. B. Schneiderman and A. Russek, Phys. Rev. 181, 311 (1969).

${ }^{19}$ M. H. Mittleman and H. Tai, Phys. Rev. A $\underline{8}, 1880$ (1973).

${ }^{20}$ W. R. Thorson and J. B. Delos, Phys. Rev. A $\underline{18}, 117$ (1978); 18, 135 (1978).

${ }^{21}$ J. Y. Park, W. Scheid, and W. Greiner, Phys. Rev. C 6, 1565 (1972); G. Terlecki, W. Scheid, H. J. Fink, and W. Greiner, ihid. 18, 265 (1978).

${ }^{22}$ (a) G. Ciocchetti, A. Molinari, Nuovo Cimento 24, 1762 (1960); Nuovo Cimento B 40, 69 (1969); (b) M. Kleber and J. Zwiegel, Phys. Rev. A 19, 579 (1979); M. Kleber and K. Unterseer, Z. Phys. A292, 311 (1979).

${ }^{23}$ P. A. Amundsen, J. Phys. B 11, 3197 (1978).

${ }^{24} \mathrm{U}$. Heinz, Ph.D. thesis, Institut für Theoretische Physik der Universität Frankfurt am Main, 1980 (unpublished).

${ }^{25}$ A. R. Edmonds, Angular Momentum in Quantum Mechanics (Princeton University Press, Princeton, N.J., 1957).
${ }^{26}$ W. Schäfer, V. Oberacker, and G. Soff, Nucl. Phys. A272, 493 (1976); W. Schäfer and G. Soff, Nuovo Cimento 31B, 250 (1976); J. Rafelski, Phys. Rev. C 13 , 2086 (1976).

${ }^{27}$ C. Gaussorgues, C. LeSech, F. Masnou-Seeuws, R. McCarroll, and A. Riera, J. Phys. B $\underline{8}, 239$ (1975).

${ }^{28}$ M. S. Child, Molecular Collision Theory (Academic, New York, 1974).

${ }^{29}$ N. Fröman and P. O. Fröman, JWKB Approximation (North-Holland, Amsterdam, 1965).

${ }^{30}$ W. Betz, Ph.D. thesis, Institut für Theoretische Physik der Universität Frankfurt am Main, 1980 (unpublished).

${ }^{31}$ Reference 27, p. 253.

${ }^{32}$ P. T. Greenland, Theor. Chim. Acta (Berlin) 42, 273 (1976); D. I. Abramov, A. Ya. Kazakov, L. I. Ponomarev, S. Yu. Slavyanov, and L. N. Somov, J. Phys. B 12,1761 (1979).

${ }^{33}(\mathrm{a})$ G. Soff, B. Müller, and W. Greiner, Proceedings of the Predeal International School, Heavy Ion Physics, 1978, Bucharest, Romania, edited by A. Berinde,

V. Ceaucescu, and I. A. Dorobantu; (b) J. Reinhardt, G. Soff, B. Müller, and W. Greiner, Prog. Part. Nucl. 4,503 (1980).

${ }^{34} \mathrm{G}$. Soff, J. Reinhardt, B. Müller, and W. Greiner, Z. Phys. A 294, 137 (1980).

${ }^{35}$ J. S. Greenberg, H. Bokemeyer, H. Ehmling, E. Grosse, D. Schwalm, and F. Bosch, Phys. Rev. Lett. 39, 1404 (1977); H.-H. Behnke, D. Liesen, S. Hagmann, P. H. Mokler, and P. Armbruster, Z. Phys. A 288, 35 (1978); D. Liesen, P. Armbruster, H.-H. Behnke, and S. Hagmann, ibid. 288, 417 (1978).

${ }^{36} \mathrm{H}$. Bokemeyer (unpublished).

${ }^{37}$ E. Berdermann, H. Bokemeyer, F. Bosch, M. Clemente, S. Güttner, P. Kienle, C. Kozhuharov, H. Krimm, B. Martin, B. Povh, K. Traxel, and Th. Walcher, Proceedings of the Deutsche Physikalische Gesellschaft spring meeting, Gent, 1979 [Verh. Dtsch. Phys. Ges. (VI) 14, 882 (1979)].

${ }^{38}$ E. M. Rose, Relativistic Electron Theory (Wiley, New York, 1961).

${ }^{39}$ A. S. Dawidow, Quantenmechanik (VEB Deutscher Verlag der Wissenschaften, Berlin, 1978), p. 21.

${ }^{40} \mathrm{E}$. Merzbacher, Quantum Mechanics (Wiley, New York, 1970), Chap. 11.2.

${ }^{41}$ W. R. Thorson and H. Levy II, Phys. Rev. 181, 230 (1969); 181, 244 (1969); 181, 252 (1969); C. F. Lebeda, W. R. Thorson, and H. Levy II, Phys. Rev. A 4, 900 (1971); V. SethuRaman, W. R. Thorson, and F. C. Lebeda, ibid. $\underline{8}, 1316$ (1973).

${ }^{42}$ K. Taulbjerg, J. Vaaben, and F. Fastrup, Phys. Rev. A 12,2325 (1975).

${ }^{43} \mathrm{~J}$. Rankin and W. R. Thorson, Phys. Rev. A $\underline{18}, 1990$ (1978).

${ }^{44} \mathrm{~J}$. Vaaben and K. Taulbjerg, in Proceedings of the Eleventh ICPEAC Kyoto, 1979, edited by K. Takayanagi and N. Oda (The Society for Atomic Research, Kyoto, 1979), p. 566, and unpublished.

${ }^{45}$ G. Soff, W. Betz, G. Heiligenthal, J. Kirsch, B. Müller, J. Reinhardt, and W. Greiner, Fizika $\underline{9}$, Suppl. 4, 721 (1977)

${ }^{46}$ W. E. Meyerhof, Phys. Rev. Lett. 31, 1341 (1973).

${ }^{47}$ K. Taulbjerg and J. S. Briggs, J. Phys. B 8,1895 (1975).

${ }^{48} \mathrm{~F}$. Bosch, D. Liesen, P. Armbruster, D. Maor, P. H. Mokler, H. Schmidt-Böcking, and R. Schuch, Z. Phys. 
A 296, 11 (1980).

${ }^{49}$ J. Reinhardt, W. Betz, P. Gärtner, J. Kirsch, U. Müller, T. de Reus, K.-H. Wietschorke, B. Müller,

W. Greiner, and G. Soff, in Proceedings of the XVIII Winter School on Physics, Bielsko-Biala, Poland, 1980 (in press).
${ }^{50}$ J. Kirsch, Ph.D. thesis, Institut für Theoretische Physik der Universität Frankfurt am Main, 1980 (unpublished).

${ }^{51}$ V. H. Ponce, J. Phys. B 12,3731 (1979).

${ }^{52}$ G. B. Schmid, J. Phys. B $\underline{12}$, 3909 (1979). 